\title{
GRB 120422A/SN 2012bz: Bridging the gap between low- and high-luminosity gamma-ray bursts ${ }^{\star}$
}

\author{
S. Schulze ${ }^{1,2,3}$, D. Malesani ${ }^{4}$, A. Cucchiara ${ }^{5}$, N. R. Tanvir ${ }^{6}$, T. Krühler ${ }^{4,20}$, A. de Ugarte Postigo ${ }^{7,4}$, G. Leloudas ${ }^{8,4}$, \\ J. Lyman ${ }^{9}$, D. Bersier ${ }^{9}$, K. Wiersema ${ }^{6}$, D. A. Perley ${ }^{10,11}$, P. Schady ${ }^{12}$, J. Gorosabel ${ }^{7,44,45}$, J. P. Anderson ${ }^{13,20}$, \\ A. J. Castro-Tirado ${ }^{7}$, S. B. Cenko ${ }^{14,15}$, A. De Cia ${ }^{16}$, L. E. Ellerbroek ${ }^{17}$, J. P. U. Fynbo ${ }^{4}$, J. Greiner ${ }^{12}$, J. Hjorth ${ }^{4}$, \\ D. A. Kann ${ }^{12,18}$, L. Kaper ${ }^{17}$, S. Klose ${ }^{18}$, A. J. Levan ${ }^{19}$, S. Martín ${ }^{20}$, P. T. O’Brien ${ }^{6}$, K. L. Page ${ }^{6}$, G. Pignata ${ }^{21}$,
} S. Rapaport ${ }^{22}$, R. Sánchez-Ramírez ${ }^{7}$, J. Sollerman ${ }^{23}$, I. A. Smith ${ }^{24}$, M. Sparre ${ }^{4}$, C. C. Thöne ${ }^{7}$, D. J. Watson ${ }^{4}$, D. Xu ${ }^{16,4}$, F. E. Bauer ${ }^{1,2,43}$, M. Bayliss 25,26 , G. Björnsson ${ }^{3}$, M. Bremer ${ }^{28}$, Z. Cano ${ }^{3}$, S. Covino ${ }^{27}$, V. D’Elia ${ }^{29,46}$, D. A. Frail ${ }^{30}$, S. Geier ${ }^{4,31}$, P. Goldoni ${ }^{32}$, O. E. Hartoog ${ }^{17}$, P. Jakobsson ${ }^{3}$, H. Korhonen ${ }^{33}$, K. Y. Lee ${ }^{23}$, B. Milvang-Jensen ${ }^{4}$, M. Nardini ${ }^{34}$, A. Nicuesa Guelbenzu ${ }^{18}$, M. Oguri ${ }^{35,36}$, S. B. Pandey ${ }^{37}$, G. Petitpas ${ }^{25}$, A. Rossi ${ }^{18}$, A. Sandberg ${ }^{23}$, S. Schmidl ${ }^{18}$, G. Tagliaferri ${ }^{27}$, R. P. J. Tilanus ${ }^{38,39}$, J. M. Winters ${ }^{28}$, D. Wright ${ }^{40}$, and E. Wuyts ${ }^{41,42}$

(Affiliations can be found after the references)

Received 8 January 2014 / Accepted 7 March 2014

\begin{abstract}
Context. At low redshift, a handful of gamma-ray bursts (GRBs) have been discovered with luminosities that are substantially lower $\left(L_{\text {iso }} \lesssim\right.$ $\left.10^{48.5} \mathrm{erg} \mathrm{s}^{-1}\right)$ than the average of more distant ones $\left(L_{\text {iso }} \gtrsim 10^{49.5} \mathrm{erg} \mathrm{s}^{-1}\right)$. It has been suggested that the properties of several low-luminosity (low- $L$ ) GRBs are due to shock break-out, as opposed to the emission from ultrarelativistic jets. This has led to much debate about how the populations are connected.

Aims. The burst at redshift $z=0.283$ from 2012 April 22 is one of the very few examples of intermediate- $L$ GRBs with a $\gamma$-ray luminosity of $L_{\text {iso }} \sim 10^{49.6-49.9} \mathrm{erg} \mathrm{s}^{-1}$ that have been detected up to now. With the robust detection of its accompanying supernova SN 2012bz, it has the potential to answer important questions on the origin of low- and high- $L$ GRBs and the GRB-SN connection.

Methods. We carried out a spectroscopy campaign using medium- and low-resolution spectrographs with 6-10-m class telescopes, which covered a time span of 37.3 days, and a multi-wavelength imaging campaign, which ranged from radio to X-ray energies over a duration of $\sim 270$ days. Furthermore, we used a tuneable filter that is centred at $\mathrm{H} \alpha$ to map star-formation in the host and the surrounding galaxies. We used these data to extract and model the properties of different radiation components and fitted the spectral energy distribution to extract the properties of the host galaxy.

Results. Modelling the light curve and spectral energy distribution from the radio to the X-rays revealed that the blast wave expanded with an initial Lorentz factor of $\Gamma_{0} \sim 50$, which is a low value in comparison to high- $L$ GRBs, and that the afterglow had an exceptionally low peak luminosity density of $\lesssim 2 \times 10^{30} \mathrm{erg} \mathrm{s}^{-1} \mathrm{~Hz}^{-1}$ in the sub-mm. Because of the weak afterglow component, we were able to recover the signature of a shock break-out in an event that was not a genuine low- $L$ GRB for the first time. At $1.4 \mathrm{hr}$ after the burst, the stellar envelope had a blackbody temperature of $k_{\mathrm{B}} T \sim 16 \mathrm{eV}$ and a radius of $\sim 7 \times 10^{13} \mathrm{~cm}$ (both in the observer frame). The accompanying SN 2012bz reached a peak luminosity of $M_{V}=-19.7 \mathrm{mag}$, which is $0.3 \mathrm{mag}$ more luminous than SN 1998bw. The synthesised nickel mass of $0.58 M_{\odot}$, ejecta mass of $5.87 M_{\odot}$, and kinetic energy of $4.10 \times 10^{52} \mathrm{erg}$ were among the highest for GRB-SNe, which makes it the most luminous spectroscopically confirmed SN to date. Nebular emission lines at the GRB location were visible, which extend from the galaxy nucleus to the explosion site. The host and the explosion site had close-to-solar metallicity. The burst occurred in an isolated star-forming region with an SFR that is $1 / 10$ of that in the galaxy's nucleus. Conclusions. While the prompt $\gamma$-ray emission points to a high- $L$ GRB, the weak afterglow and the low $\Gamma_{0}$ were very atypical for such a burst. Moreover, the detection of the shock break-out signature is a new quality for high- $L$ GRBs. So far, shock break-outs were exclusively detected for low- $L$ GRBs, while GRB 120422A had an intermediate $L_{\text {iso }}$ of $\sim 10^{49.6-49.9} \mathrm{erg} \mathrm{s}^{-1}$. Therefore, we conclude that GRB 120422A was a transition object between low- and high- $L$ GRBs, which supports the failed-jet model that connects low- $L$ GRBs that are driven by shock break-outs and high- $L$ GRBs that are powered by ultra-relativistic jets.
\end{abstract}

Key words. gamma-ray burst: individual: GRB 120422A - supernovae: individual: SN 2012bz - dust, extinction - galaxies: ISM galaxies: individual: GRB 120422A

\section{Introduction}

The discovery of SN 1998bw in the errorbox of GRB 980425 by Galama et al. (1998) gave the study of the gamma-ray burst (GRB) - supernova (SN) connection a flying start. This event remains unique in several ways, among the many hundred GRBs that have been studied since. It is still the closest GRB with

^ Appendices are available in electronic form at http: //www . aanda.org a measured redshift, and it is the least energetic GRB yet observed. Nevertheless, SN 1998bw seems to be representative for the type of SNe that accompanies the more typical and brighter long-duration GRBs (For recent reviews, see Woosley \& Bloom 2006; Modjaz 2011; Hjorth \& Bloom 2012), which are bright ( $M_{\text {bol, peak }} \lesssim-19 \mathrm{mag}$ ), broad-lined (indicating expansion velocities of several $10^{4} \mathrm{~km} \mathrm{~s}^{-1}$ ) type Ic $\mathrm{SNe}$ (i.e. lacking hydrogen and helium). Interestingly, in only two out of 16 cases of nearby long-duration GRBs $(z<0.5)$, no SN was found to limits several 
magnitudes deeper than any other GRB-SN. (Fynbo et al. 2006; Della Valle et al. 2006a; Gal-Yam et al. 2006; Ofek et al. 2007; Kann et al. 2011), though their classification is not free of ambiguity (e.g. McBreen et al. 2008; Thöne et al. 2008; Zhang et al. 2009; Kann et al. 2011).

So far, most GRBs with spectroscopically-confirmed SN associations have had a much lower apparent luminosity than the bulk of long-duration GRBs. The GRB 030329 was the first example of an high-luminosity GRB $\left(\log L_{\text {iso }} /\left(\mathrm{erg} \mathrm{s}^{-1}\right)=\right.$ 50.9) that was accompanied by an SN (Hjorth et al. 2003; Matheson et al. 2003; Stanek et al. 2003). However, there is a growing number of high-luminosity bursts, which are defined by $\log L_{\text {iso }} /\left(\mathrm{erg} \mathrm{s}^{-1}\right) \gtrsim 49.5$ (Hjorth 2013), with a spectroscopically-confirmed $\mathrm{SN}$, such as GRBs 050525A (Della Valle et al. 2006b), 081007 (Della Valle et al. 2008; Jin et al. 2013), 091127 (Cobb et al. 2010; Berger et al. 2011), 101219B (Sparre et al. 2011), 130215A (de Ugarte Postigo et al. 2013), 130427 A (Xu et al. 2013; Levan et al. 2013), and 130831A (Klose et al. 2013).

Bromberg et al. (2011) suggested that low-luminosity GRBs, such as GRBs 060218 and $100316 \mathrm{D},\left(\log L_{\text {iso }} /\left(\mathrm{erg} \mathrm{s}^{-1}\right) \lesssim 48.5\right.$; Hjorth 2013) are driven by a high-energy emission that is associated with the shock break-out of their progenitor stars rather (see also Nakar \& Sari 2012) than an emerging jet that is typical in high-luminosity GRBs (Colgate \& McKee 1969; Kulkarni et al. 1998; Campana et al. 2006; Soderberg et al. 2006a; Nakar $\&$ Sari 2012; Olivares et al. 2012). A consequence of these different energy sources is that low- $L$ GRBs seem to be about 10-1000 times more common than high- $L$ GRBs (Pian et al. 2006; Chapman et al. 2007; Guetta \& Della Valle 2007; Liang et al. 2007; Virgili et al. 2009; Wanderman \& Piran 2010). Because of their low luminosities, however, they are primarily found at low redshifts as rare events (one every $\sim 3$ years). In contrast to high- $L$ GRBs, low- $L$ GRBs typically have single-peak high-energy prompt light curves and can have soft high-energy spectra with peak energies below $\sim 50 \mathrm{keV}$ (Campana et al. 2006; Starling et al. 2011, but see Kaneko et al. 2007). Their optical emission is dominated by the SN emission. Until now, their afterglows have been detected only in radio and X-rays but not in optical. The recent GRB 120422A is a particularly interesting case. It has a $\gamma$-ray luminosity that is intermediate between low- and high-luminosity GRBs and has a robust detection of the associated SN (Malesani et al. 2012a; Sánchez-Ramírez et al. 2012; Wiersema et al. 2012; Melandri et al. 2012). A study of this event may thus answer important questions about the origin of both high- and low- $L$ GRBs.

The paper is structured as follows. We describe the data gathering and outline the data analysis in Sect. 2. We then present the results on the transient following the GRB from radio to X-ray wavelengths and the accompanying GRB-SN, SN 2012bz, in Sect. 3. The properties of the GRB environment and the host galaxy are described in Sect. 4. In Sect. 5, we compare our findings to other events and argue that GRB 120422A represents the missing link between low- and high-L GRBs. Finally, we summarise our findings and present our conclusions in Sect. 6.

Throughout the paper, we use the convention for the flux density $F_{v}(t) \propto t^{-\alpha} v^{-\beta}$, where $\alpha$ is the temporal slope and $\beta$ is the spectral slope. We refer to the solar abundance compiled in Asplund et al. (2009) and adopt $\mathrm{cm}^{-2}$ as the linear unit of column densities, $N$. Magnitudes reported in the paper are given in the $\mathrm{AB}$ system, and uncertainties are given at an $1 \sigma$ confidence level (c.l.). We assume a $\Lambda$ CDM cosmology with $H_{0}=71 \mathrm{~km} \mathrm{~s}^{-1} \mathrm{Mpc}^{-1}, \Omega_{\mathrm{m}}=0.27$, and $\Omega_{\Lambda}=0.73$ (Larson et al. 2011).

\section{Observations and data reduction}

On 2012 April 22 at 7:12:49 UTC (hereafter called $T_{0} ; \mathrm{MJD}=56039.30057$ ), the Burst Alert Telescope (BAT, Barthelmy et al. 2005) aboard Swift detected and localised a faint burst (Troja et al. 2012). Its $\gamma$-ray light curve was comprised of a single peak with a duration of $T_{90}=5.4 \pm 1.4 \mathrm{~s}$, followed by a fainter and lower-energetic emission that began $45 \mathrm{~s}$ after the trigger and lasted for $20 \mathrm{~s}$. Within $86 \mathrm{~s}$, the Swift X-ray Telescope XRT (Burrows et al. 2005) and the UV/Optical Telescope UVOT (Roming et al. 2005) started to observe the field and detected an uncatalogued and rapidly decaying source at RA, Dec $(J 2000)=09^{\mathrm{h}} 07^{\mathrm{m}} 38^{\mathrm{s}} 42( \pm 0.01),+14^{\circ} 01^{\prime} 07^{\prime \prime} .1( \pm 0.2)$ (Beardmore et al. 2012; Kuin \& Troja 2012; Zauderer et al. 2012). At only $2^{\prime \prime} \mathrm{NE}$ of the explosion site, there is a SDSS galaxy (Cucchiara et al. 2012; Tanvir et al. 2012). Spectra of the explosion site revealed several absorption and emission lines at a common redshift of $z=0.283$, and a large number of emission lines at the location of the SDSS galaxy at a redshift identical to that of the GRB (Schulze et al. 2012b; Tanvir et al. 2012).

Thanks to its low redshift and its $\gamma$-ray luminosity $\left(E_{\text {iso }}=\right.$ $(1.6-3.2) \times 10^{50} \mathrm{erg}$ and $L_{\text {iso }} \sim 10^{49.6-49.9} \mathrm{erg} \mathrm{s}^{-1}$ measured between $1 \mathrm{keV}$ and $1000 \mathrm{keV}$; Melandri et al. 2012), which is between that of high- and low- $L$ GRBs, it is an ideal target to search for the accompanying GRB-SN. We therefore triggered an extensive imaging campaign with several telescopes from $\mathrm{mm}$ to optical wavelengths, as well as a large low- and medium-resolution spectroscopy campaign carried out at 6-m to 10 -m class telescopes. These campaigns began $\sim 31$ min after the trigger and ended $\sim 44.6$ days later. Furthermore, we obtained an X-ray spectrum with XMM-Newton 12 days after the explosion. In addition to our own efforts, the GRB-dedicated satellite Swift observed the GRB at UV/optical and X-ray wavelengths for 54.3 days. We incorporated these data and the radio data obtained with the Arcminute Microkelvin Imager Large Array (AMI-LA; Staley et al. 2013) to present a comprehensive study of this event. In the following, we summarise the observations and describe how the data were analysed. A log of our observations is presented in Tables 1, 2, A.1, and B.1.

\subsection{Optical and NIR spectroscopy}

Our spectroscopic campaign began $51 \mathrm{~min}$ after the trigger and covered a time span of 37.7 days. The spectral sequence was comprised of seven medium-resolution spectra obtained with VLT/X-shooter (Vernet et al. 2011); the first three spectra covered the full spectral bandwidth from 3000 to $24800 \AA$, while a $K$-blocking filter (cutting the wavelength coverage at $20700 \AA$; Vernet et al. 2011) was adopted to increase the signal-to-noise ratio $(\mathrm{S} / \mathrm{N})$ in the $H$ band for the remaining ones. These observations were complemented with ten low-resolution spectra acquired with the Gemini Multi-Object Spectrograph (GMOS, Hook et al. 2004), which is mounted on Gemini-North and -South, the Gran Telescopio Canarias (GTC) OSIRIS camera, the Keck Low Resolution Imaging Spectrometer (LRIS; Oke et al. 1995), and the Magellan Low Dispersion Survey Spectrograph 3 (LDSS3). Table 1 summarises these observations.

Observing conditions were not always photometric, and observations were performed irrespective of moon distance and phase. For each epoch, we centred the slit on the explosion site and varied the position angle to probe different parts of the host galaxy in some cases, as illustrated in Fig. 1. 
Table 1. Summary of spectroscopic observations.

\begin{tabular}{|c|c|c|c|c|c|c|c|c|}
\hline $\begin{array}{l}\text { MJD } \\
\text { (days) }\end{array}$ & $\begin{array}{l}\text { Epoch } \\
\text { (days) }\end{array}$ & Telescope/Instrument & Arm/Grating & $\begin{array}{c}\text { Spectral } \\
\text { range }(\AA)\end{array}$ & $\begin{array}{c}\text { Resolving } \\
\text { power }\end{array}$ & $\begin{array}{l}\text { Exposure } \\
\text { time }(\mathrm{s})\end{array}$ & $\begin{array}{c}\text { Slit } \\
\text { width }\end{array}$ & $\begin{array}{c}\text { Position } \\
\text { angle }\end{array}$ \\
\hline 56039.345 & 0.0443 & Gemini/GMOS-N & R400+OG515 & $5942-10000$ & 960 & $2 \times 900$ & 1.0 & $180^{\circ} 0$ \\
\hline 56039.431 & 0.1301 & Gemini/GMOS-N & B600 & $3868-6632$ & 844 & $2 \times 400$ & 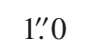 & $180^{\circ} 0$ \\
\hline 56040.017 & 0.7160 & VLT/X-shooter & $\begin{array}{l}\text { UVB } \\
\text { VIS } \\
\text { NIR }\end{array}$ & $\begin{array}{c}3000-5500 \\
5500-10000 \\
10000-24800\end{array}$ & $\begin{array}{l}4350 \\
8800 \\
5100\end{array}$ & $\begin{array}{l}4 \times 1200 \\
4 \times 1200 \\
16 \times 300\end{array}$ & $\begin{array}{l}1.90 \\
0.9 \\
0.9\end{array}$ & $41: 0$ \\
\hline 56042.911 & 3.6112 & GTC/OSIRIS & R500R & $4800-10000$ & 500 & $4 \times 1500$ & 1.2 & 100.0 \\
\hline 56044.014 & 4.7139 & VLT & $\begin{array}{l}\text { UVB } \\
\text { VIS } \\
\text { NIR }\end{array}$ & $\begin{array}{c}3000-5500 \\
5500-10000 \\
10000-24800\end{array}$ & $\begin{array}{l}4350 \\
8800 \\
5100\end{array}$ & $\begin{array}{l}4 \times 1200 \\
4 \times 1200 \\
16 \times 300\end{array}$ & $\begin{array}{l}1.9 \\
1.9 \\
0 ! 9 \\
0.9\end{array}$ & $41^{\circ} 0$ \\
\hline 56044.257 & 4.9565 & Keck/LRIS & $\begin{array}{l}400 / 3400 \\
400 / 8500\end{array}$ & $\begin{array}{c}3000-5500 \\
5500-10000\end{array}$ & $\begin{array}{c}750 \\
1700\end{array}$ & $2 \times 900$ & 0.7 & 50.0 \\
\hline 56048.061 & 8.7604 & VLT/X-shooter & $\begin{array}{l}\text { VIS } \\
\text { NIR }\end{array}$ & $\begin{array}{c}3000-5500 \\
5500-10000 \\
10000-24800\end{array}$ & $\begin{array}{l}4350 \\
8800 \\
5100\end{array}$ & $\begin{array}{l}4 \times 1200 \\
4 \times 1200 \\
16 \times 300\end{array}$ & $\begin{array}{l}1.9 \\
0 ! 9 \\
0.9\end{array}$ & $41^{\circ} 0$ \\
\hline 56048.304 & 9.0036 & Gemini/GMOS-N & R400 & $4442-8608$ & 960 & $4 \times 1200$ & $1 . .0$ & 170.0 \\
\hline 56052.978 & 13.6772 & Gemini/GMOS-S & R400+GG455 & 4892-9008 & 960 & $1 \times 2400$ & 1.0 & $180^{\circ} 0$ \\
\hline 56053.930 & 14.6301 & GTC/OSIRIS & R500R & $4800-10000$ & 500 & $3 \times 1200$ & $1 . " 2$ & $75^{\circ} 0$ \\
\hline 56057.996 & 18.6962 & VLT/X-shooter $^{a}$ & $\begin{array}{l}\text { VIS } \\
\text { NIR }\end{array}$ & $\begin{array}{c}3000-5500 \\
5500-10000 \\
10000-20700\end{array}$ & $\begin{array}{l}4350 \\
8800 \\
5100\end{array}$ & $\begin{array}{l}4 \times 1200 \\
4 \times 1200 \\
16 \times 300\end{array}$ & $\begin{array}{l}1.90 \\
0 ! 9 \\
0.9\end{array}$ & $52^{\circ} .0$ \\
\hline 56061.996 & 22.6953 & Gemini/GMOS-S & R400+GG455 & 4892-9108 & 960 & $2 \times 2400$ & 1.0 & -30.0 \\
\hline 56063.999 & 24.6992 & VLT/X-shooter ${ }^{a}$ & $\begin{array}{l}\text { UVB } \\
\text { VIS } \\
\text { NIR }\end{array}$ & $\begin{array}{c}3000-5500 \\
5500-10000 \\
10000-20700\end{array}$ & $\begin{array}{l}4350 \\
8800 \\
5100\end{array}$ & $\begin{array}{l}4 \times 1200 \\
4 \times 1200 \\
16 \times 300\end{array}$ & $\begin{array}{l}1.9 \\
0 ! 9 \\
0.9\end{array}$ & 52.0 \\
\hline 56066.068 & 26.7680 & Magellan/LDSS3 & VPH_ALL & $3700-9400$ & 800 & $1 \times 1400$ & 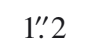 & $141^{\circ} .0$ \\
\hline 56076.025 & 36.7250 & VLT/X-shooter ${ }^{a}$ & $\begin{array}{l}\text { UVB } \\
\text { VIS } \\
\text { NIR }\end{array}$ & $\begin{array}{c}3000-5500 \\
5500-10000 \\
10000-20700\end{array}$ & $\begin{array}{l}4350 \\
8800 \\
5100\end{array}$ & $\begin{array}{l}4 \times 1200 \\
4 \times 1200 \\
16 \times 300\end{array}$ & $\begin{array}{l}1.90 \\
0 ! 9 \\
0.9\end{array}$ & -143.9 \\
\hline 56077.000 & 37.7001 & VLT/X-shooter ${ }^{a}$ & $\begin{array}{l}\text { UVB } \\
\text { VIS } \\
\text { NIR }\end{array}$ & $\begin{array}{c}3000-5500 \\
5500-10000 \\
10000-20700\end{array}$ & $\begin{array}{l}4350 \\
8800 \\
5100 \\
\end{array}$ & $\begin{array}{l}4 \times 1200 \\
4 \times 1200 \\
16 \times 300\end{array}$ & $\begin{array}{l}1 . .0 \\
0 ! 9 \\
0 ! 9\end{array}$ & $151^{\circ} .1$ \\
\hline
\end{tabular}

Notes. Column "Epoch" shows the logarithmic mean-time after the burst in the observer frame. Resolving powers and spectral ranges are the nominal values from instrument manuals. ${ }^{(a)}$ The $K$-band blocking filter was used to increase the $\mathrm{S} / \mathrm{N}$ in $J H$ band.

The VLT/X-shooter data were reduced with the X-shooter pipeline v2.0 (Goldoni et al. 2006) ${ }^{1}$. To extract the onedimensional spectra of the transient and the host galaxy, we used a customised tool that adopts the optimal extraction algorithm by Horne (1986). The Gemini, GTC, and Magellan spectra were reduced and calibrated using standard procedures in IRAF (Tody 1993). The Keck data were reduced with a custom pipeline that makes use of standard techniques of long-slit spectroscopy. In all cases, we chose a small aperture for studying the optical transient. For studying the emission lines, we extracted the spectral point spread (PSF) function and extracted the spectrum of the nucleus and the afterglow within an aperture of $1 \times F W H M$ of each trace, for example, the FWHMs were 1".34 for the galaxy nucleus and 0.'86 for the explosion site, for the UVB and VIS of the first X-shooter spectrum.

All spectra were flux-calibrated with corresponding spectrophotometric standard star observations, and the absolute flux scale was adjusted by comparing to photometry. The data were corrected for the Galactic reddening of $E(B-V)=0.04 \mathrm{mag}$ (Schlegel et al. 1998). All wavelengths were transformed to vacuum wavelengths. In addition, X-shooter data were corrected for

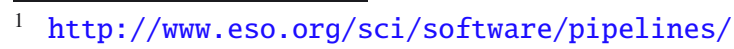

heliocentric motion. No telluric correction was applied, as it has no implications for our analysis.

\subsection{Imaging}

Following the BAT trigger, Swift slewed immediately to the burst, and UVOT took a $v$-band settling exposure $86 \mathrm{~s}$ after the BAT trigger. Science observations began at $T_{0}+104 \mathrm{~s}$ and cycled through all filters. Follow-up observations in the $v$ and $b$ bands continued until $T_{0}+2.3$ days, in the $u v w 1$, uvm 2 , and $u v w 2 \mathrm{UV}$ filters until $T_{0}+9.7$ days, and in the $u$ band until $T_{0}+54.3$ days, at which time a final set of observations of the host galaxy was taken in all filters ${ }^{2}$.

Our ground-based imaging campaign began $31 \mathrm{~min}$ after the explosion and spanned a time interval of $\sim 45$ days. Due to the proximity of an $R=8.24 \mathrm{mag}$ star $\left(79^{\prime \prime} \mathrm{NW}\right.$ of the explosion site), we either moved the position of the optical transient to the NW corner of the chip or (most of the time) obtained short dithered exposures to avoid excessive saturation.

\footnotetext{
2 Additional UVOT data were acquired in October 2012. These data are not discussed in this paper. This has no implications on our work.
} 
Table 2. Summary of $\mathrm{mm}$ and sub-mm observations.

\begin{tabular}{cccccc}
\hline \hline $\begin{array}{c}\text { MJD } \\
\text { days })\end{array}$ & $\begin{array}{c}\text { Epoch } \\
\text { (days) }\end{array}$ & Instrument & Frequency & $\begin{array}{c}\text { Exposure } \\
\text { time }(\mathrm{s})\end{array}$ & $\begin{array}{c}F_{v} \\
(\mathrm{mJy} ; 3 \sigma)\end{array}$ \\
\hline 56039.3291 & 0.0537 & SCUBA-2 & $350 \mathrm{GHz}$ & 5639 & $<7.20$ \\
56039.3291 & 0.0537 & SCUBA-2 $^{2}$ & $665 \mathrm{GHz}$ & 5639 & $<225$ \\
56039.5676 & 0.2670 & AMI-LA $^{a}$ & $15 \mathrm{GHz}$ & & $<0.62$ \\
56040.1923 & 0.8917 & SMA $^{a}$ & $272 \mathrm{GHz}$ & 3420 & $<3.60$ \\
56041.6806 & 2.3800 & AMI-LA $^{a}$ & $15 \mathrm{GHz}$ & & $<0.47$ \\
56041.9422 & 2.6416 & PdBI & $86.7 \mathrm{GHz}$ & 5040 & $<0.39$ \\
56041.9943 & 2.6937 & CARMA $^{a}$ & $92.5 \mathrm{GHz}$ & 3480 & $<1.15$ \\
56043.6806 & 4.3800 & AMI-LA $^{a}$ & $15 \mathrm{GHz}$ & & $<0.37$ \\
56046.7206 & 7.4200 & AMI-LA $^{a}$ & $15 \mathrm{GHz}$ & & $<0.24$ \\
56048.8054 & 9.5048 & PdBI $^{a}$ & $86.7 \mathrm{GHz}$ & 5040 & $<0.24$ \\
56052.7506 & 13.450 & AMI-LA $^{a}$ & $15 \mathrm{GHz}$ & & $<0.23$ \\
56067.8906 & 28.590 & AMI-LA $^{a}$ & $15 \mathrm{GHz}$ & & $<0.46$ \\
\hline
\end{tabular}

Notes. Column "Epoch" shows the logarithmic mean time after the burst in the observer frame. ${ }^{(a)}$ Data taken from Staley et al. (2013).

Observations were carried out with the 2.56-m Nordic Optical Telescope (NOT) equipped with ALFOSC, MOSCA, and StanCAM in the $u^{\prime} g^{\prime} R r^{\prime} I i^{\prime}$ bands (Malesani et al. 2012b; Schulze et al. 2012a). These observations began at $14.29 \mathrm{~h}$ postburst and were stopped at 44.5 days because of the small Sun distance. Further imaging data were acquired with GMOS-N and GMOS-S in the $u^{\prime} g^{\prime} r^{\prime} i^{\prime} z^{\prime}$ bands between $31 \mathrm{~min}$ and 40.7 days after the explosion (Cucchiara et al. 2012; Perley et al. 2012a). The Gamma-Ray Optical/Near-infrared Detector (GROND, Greiner et al. 2007, 2008) mounted at the MPG/ESO $2.2 \mathrm{~m}$ telescope on La Silla imaged the field simultaneously in four optical $\left(g^{\prime} r^{\prime} i^{\prime} z^{\prime}\right)$ and three NIR $\left(J H K_{\mathrm{s}}\right)$ bands starting at $T_{0}+16.5 \mathrm{hr}$ (Nardini et al. 2012). Additional epochs were obtained on nights $2,9,11,20$, and 29 before the visibility of the field was compromised by its small Sun distance on day 39. We monitored the optical transient in the $g^{\prime} r^{\prime} i^{\prime}$ bands with the 60-inch Palomar telescope for 37 days beginning at $T_{0}+0.87$ day and in the $J H K$ bands with the Wide Field Camera (WFCAM) mounted at the United Kingdom Infrared Telescope (UKIRT) on Mauna Kea at seven epochs between $T_{0}+0.06$ day and $T_{0}+25.98$ day.

We complemented these optical observations with the 10.4-m GTC telescope equipped with OSIRIS in the $g^{\prime} r^{\prime} i^{\prime} z^{\prime}$ bands, the multi-filter imager BUSCA mounted at the 2.2-m telescope of Calar Alto (CAHA) in $g^{\prime}$ and the $r^{\prime}$ bands $^{3}$, the $3.5-\mathrm{m}$ CAHA telescope equipped with the Omega 2000 camera in the $z^{\prime}$ band ${ }^{4}$, the LDSS3 camera mounted at the 6-m Clay telescope telescope in the $r^{\prime}$ and $i^{\prime}$ bands, the Direct CCD Camera mounted on the Irenee du Pont 2.5-m telescope at Las Campanas in the $r^{\prime}$ and $i^{\prime}$ bands, the 2.4-m Gao-Mei-Gu (GMG) telescope in $i^{\prime}$, and the 1.04-m and the 2-m optical-infrared Himalayan Chandra Telescope in $R_{\mathrm{c}}$ and $I_{\mathrm{c}}$. Additional NIR data were acquired with the Omega 2000 in the $Y J H K_{\mathrm{s}}$ bands, the Near-InfraRed Imager (NIRI) mounted on Gemini-North in the $J$ and $K$ bands, and the Wide-field Infrared Camera (WIRC) on the 200-inch Hale telescope at Palomar Observatory in the $J$ band (Perley et al. 2012b).

Very late-time observations were secured with the $2.0-\mathrm{m}$ Liverpool telescope, with BUSCA mounted at the 2.2-m CAHA, and GMOS mounted at Gemini-North (Table B.1). The observation with the Liverpool telescope comprises 185 images.

\footnotetext{
3 http://www. caha.es/newsletter/news01a/busca/

4 http://www .mpia-hd.mpg.de/IRCAM/02000/
}

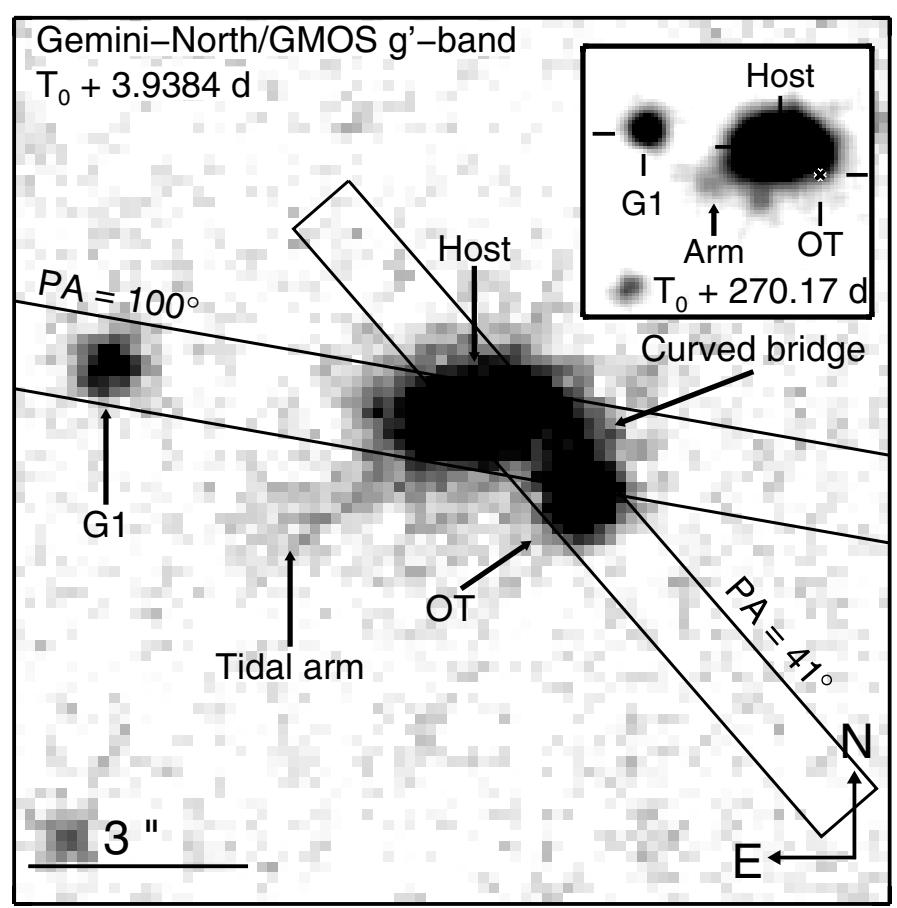

Fig. 1. Field of GRB 120422A $\left(12^{\prime \prime} \times 12^{\prime \prime}\right)$. The position of the optical transient (OT) accompanying GRB 120422A is marked, as well as the host galaxy and the curved bridge of emission that connects the explosion site with the host's nucleus. Galaxy G1 has the same redshift as the GRB. The projected distance between the explosion site and the galaxy G1 is $28.7 \mathrm{kpc}$. The inset shows the field observed in the $g^{\prime}$ band with GMOS-N at 270.2 days after the burst. The image cuts were optimised to increase the visibility of the tidal arm that partly connects the host galaxy and G1. The most important slit orientations of our spectroscopic campaign (Table 1) are overlaid.

To minimise the data heterogeneity, an observational seeing constraint of $<1$ ". 1 was imposed for all epochs. The CAHA observation did, unfortunately, not go very deep. We do not discuss these data in the following.

In addition to these broadband observations, we made use of the tuneable filters at the 10.4-m GTC to trace the $\mathrm{H} \alpha$ emission in the host galaxy on 2012 May 16, which was 25.5 days after the burst. Observations consisted of $5 \times 600 \mathrm{~s}$ exposures using a $15-\AA$ wide filter tuned to the wavelength of $\mathrm{H} \alpha$ at the redshift of the burst $\left(\lambda_{\mathrm{obs}}=8420 \AA\right)$ and a $3 \times 100 \mathrm{~s}$ exposure with a 513 - $\AA$-wide order-sorter filter centred at $8020 \AA$ to probe the continuum emission (filter f802/51). The seeing was $\sim 1^{\prime \prime}$, although the transparency was affected by extinction due to Saharan dust suspended in the atmosphere (Calima).

In general, observing conditions were not always photometric; in particular, part of the NOT observations suffered from poor transparency due to the Calima. Table A.1 summarises all observations with good data quality.

We obtained the UVOT data from the Swift Data Archive ${ }^{5}$. These data had bad pixels identified, were mod-8 noise corrected, and are endowed with FK5 coordinates. We used the standard UVOT data analysis software distributed with HEASOFT 6.12 along with the standard calibration data ${ }^{6}$. Optical and NIR data were processed through standard procedures (bias subtraction and flat field normalisation) using

\footnotetext{
5 http://www.swift.ac.uk/swift_portal/

6 http://heasarc.nasa.gov/lheasoft/
} 
IRAF or instrument-specific software packages, which include the GEMINI IRAF software package for GMOS and NIRI, a customised pipeline for the GROND data (for details, we refer to Yoldaş et al. 2008 and Krühler et al. 2008), a modified version of the WIRCSoft package for P200/WIRC data ${ }^{7}$, and the UKIRT pipeline for the WFCAM data ${ }^{8}$. Some observations suffered from variable conditions, and in those cases, individual images were weighted according to their S/N. The $i^{\prime}$ - and $z^{\prime}$-band images suffer from fringing, which were corrected using a fringe pattern computed from the science data themselves, although the presence of the halo from the nearby bright star hampered the process in some cases. These data resulted in a lower $\mathrm{S} / \mathrm{N}$. Astrometric calibration was computed against the USNO-B1 catalog (Monet et al. 2003), yielding an RMS of 0.4 . All images were then registered together, yielding a relative RMS of less than 0.'08. We measure the afterglow location to be RA, Dec $(\mathrm{J} 2000)=09^{\mathrm{h}} 07^{\mathrm{m}} 38^{\mathrm{s}} 42,+14^{\circ} 01^{\prime} 07^{\prime \prime} .5$.

\subsubsection{Sub- $\mathrm{mm} / \mathrm{mm}$ observations}

Our sub-mm/mm observations comprise of five epochs and cover a time interval of 9.48 days. First, Smith et al. (2012) simultaneously obtained an early epoch at $450 \mu \mathrm{m}$ and $850 \mu \mathrm{m}$ with the sub-millimetre continuum camera SCUBA-2 (Holland et al. 2013) on the James Clerk Maxwell Telescope (JCMT). The 1.6-h observation began at $T_{0}+41.5 \mathrm{~min}$ and was performed under moderate weather conditions. The CSO $225 \mathrm{GHz}$ tau, which measures the zenith atmospheric attenuation, was 0.089 initially but generally degraded through the run. The elevation of GRB 120422A fell from 54.6 to 30.4. In the consecutive night, Martin et al. (2012) triggered a short 45-min snapshot observation at the Submillimeter Array (SMA) at $T_{0}+21.4 \mathrm{hr}$. Receivers were tuned to the local oscillator (LO) centre frequency of $271.8 \mathrm{GHz}(\lambda=1.1 \mathrm{~mm})$ with the correlator configured to cover two $4-\mathrm{GHz}$ bands centred at $\pm 6 \mathrm{GHz}$ from the LO frequency. All 8 SMA antennas were used in its very extended configuration under excellent weather conditions with an average zenith opacity of 0.03 (precipitable water vapour of PWV $\sim 0.5 \mathrm{~mm}$ ) at $225 \mathrm{GHz}$. A further observation was carried out by Perley (2012) with the Combined Array for Research in Millimeter-Wave Astronomy (CARMA) in D-configuration at $92.5 \mathrm{GHz}(\lambda=3 \mathrm{~mm})$. This observation was carried out between 23:13 UT on 24 April and 00:29 UT on April 25. The total on-source integration time was $58 \mathrm{~min}$. We finally obtained two epochs with the Plateau de Bure Interferometer (PdBI) at a frequency of $86.7 \mathrm{GHz}(\lambda=3.4 \mathrm{~mm})$ in its six-antenna compact D configuration. These observations began at $T_{0}+2.6416$ and 9.5048 days and lasted for $84 \mathrm{~min}$ each. The AMI-LA observed at six epochs between 0.27 and 28.59 days after the burst (Staley et al. 2013).

The SCUBA-2 data were reduced in the standard manner (Chapin et al. 2013) using SMURF (version 1.5.0) and KAPPA (version 2.1-4) from the Starlink Project ${ }^{9}$. Observations of the SCUBA-2 calibrator Mars bracketed the GRB 120422A observation, and observations of the calibrator CRL2688 were taken several hours later. The calibration observations spanned a larger range of weather conditions than that during the GRB 120422A run and generally agreed with the standard values of the flux conversion factors (Dempsey et al. 2013), which were then used

\footnotetext{
7 http://humu.ipac.caltech.edu/ jason/sci/wircsoft/ index.html

8 http://casu.ast. cam.ac.uk/surveys-projects/wfcam

9 http://starlink. jach.hawaii.edu/starlink
}

for the flux normalisation. We reduced CARMA and SMA data with the MIRIAD and MIR-IDL software packages (Sault et al. $1995)^{10}$. The CARMA data were absolute flux calibrated with observations of 3C84 and Mars. The calibration of the SMA data is twofold: first, we used the nearby quasars J0854+201 and J0909+013 as atmospheric gain calibrators and then J0854+201 for bandpass calibration. Absolute flux calibration was bootstrapped from previous measurements of these quasars, which resulted in an absolute flux uncertainty of $\sim 30 \%$. The PdBI data were reduced with the standard CLIC and MAPPING software distributed by the Grenoble GILDAS group ${ }^{11}$. The flux calibration was secured with the Be binary star system MWC349 $\left(F_{v}=1.1 \mathrm{Jy}\right.$ at $\left.86.7 \mathrm{GHz}\right)$.

\subsubsection{X-ray observations}

The X-ray telescope (XRT) aboard Swift started to observe the BAT GRB error circle roughly $90 \mathrm{~s}$ after the trigger, while it was still slewing. Observations were first carried out in window timing (WT) mode for $80 \mathrm{~s}$. When the count rate was $\lesssim 1 \mathrm{ct} \mathrm{s}^{-1}$, the XRT switched to photon counting (PC) mode. Observations continued until $T_{0}+53.8$ days, when the visibility of the field was compromised by its small Sun distance. We obtained the temporal and spectroscopic data from the Swift/XRT Light Curve and Spectrum Repository (Evans et al. 2007, 2009). GRB 120422A was also observed by XMM-Newton under a DDT proposal, starting at 2012 May 3, 15:13 UT. At this epoch, exposures of 56841, 58421, and 58426 s were obtained with the PN, MOS1, and MOS2 detectors, respectively.

To analyse the spectroscopic data, we used Xspec, version 12.7.1, as part of HeaSoft 6.12 and the respective calibration files for XMM-Newton and Swift/XRT. The X-ray emission up to $T_{0}+200 \mathrm{~s}$ was discussed in detail in Starling et al. (2012) and Zhang et al. (2012). Therefore, we focus on the analysis of the data after that epoch. In total, XRT registered 270 background-subtracted photons between 0.3 and $10 \mathrm{keV}$; data that were flagged as bad were excluded from analysis. We re-binned the spectrum to have at least 20 count per bin and applied $\chi^{2}$ statistics.

\subsection{Photometry}

Measuring the brightness of the transient is complicated due to blending with its extended, offset host galaxy. To limit the contribution of the host to the photometry of the transient photometry, we used PSF fitting techniques. Using bright field stars, a model of the PSF was constructed for each individual image and fitted to the optical transient. To provide reliable fit results, all images were registered astrometrically to a precision that is greater than 0 .'08, and the centroid of the fitted PSF was held fixed to the position of the optical transient with a small margin of re-centring that corresponds to the uncertainty of the astrometric alignment of the individual images. In addition, the PSF-fitting radius was adjusted to the specific conditions of the observations and instrument, in particular, to the seeing and pixel scale. The fit radius is different for each observation but is typically in the range between 0.5 and 0.8 . Generally, the radius was smaller under unfavourable sky conditions in an attempt to minimise the host's effect on the fit. Naturally, this leads to a lower $\mathrm{S} / \mathrm{N}$ for these measurements than one would expect for isolated point sources.

\footnotetext{
10 http://www.atnf.csiro.au/computing/software/miriad/ https://www. cfa.harvard. edu/ cqi/mircook.html

${ }^{11}$ http://wWw.iram.fr/IRAMFR/GILDAS
} 
For images taken under adverse sky conditions (seeing $\gtrsim 1$.' 6 ) with either imagers with large pixel scales (e.g. the NIR channels of GROND with 0.'6 per pixel) or filters/epochs with low $\mathrm{S} / \mathrm{N}$ (e.g. most of the late NIR data), the individual contributions of point-source and galaxy cannot be disentangled robustly. These measurements are ignored in the following analysis. For all observations, the source was close to the centre of the field of view, and differences in the PSF between observations were, therefore, negligible.

To measure the brightness of the transient in the UVOT images, we measured the host galaxy flux at the position of the SN from the later UVOT observations, where there was no longer a contribution from the GRB or SN. This additional flux was then subtracted from our photometric measurements at the position of the GRB. In contrast, host-galaxy photometry was performed via aperture techniques. Here, we used our PSF-model to subtract the transient from the deepest images in each filter with the clearest separation between galaxy and point source, which are those images with the smallest full width at half maximum (FWHM) of the stellar PSF. A circular aperture radius was chosen to be sufficiently large $\left(2{ }^{\prime \prime} 5\right.$, e.g. $10.7 \mathrm{kpc}$ at $\left.z=0.2825\right)$, so that the missed emission from low surface brightness regions does not affect our photometry significantly. In addition, we also corroborated the galaxy photometry using elliptical Kron apertures (Kron 1980) via their implementation in Source Extractor (Bertin \& Arnouts 1996).

Once an instrumental magnitude was established, it was photometrically calibrated against the brightness of a number of field stars measured in a similar manner. Photometry was tied to the SDSS DR8 (Aihara et al. 2011) in the optical filters $\left(u^{\prime} g^{\prime} r^{\prime} i^{\prime} z^{\prime}\right)$ and 2MASS (Skrutskie et al. 2006) in the NIR $\left(J H K_{\mathrm{s}}\right)$. For those filter bands not covered by our primary calibration systems (e.g. $I_{\mathrm{C}}$ or $Y$ ), we used the instrument-specific band passes to transform magnitudes into the respective filter system via synthetic photometry, which is similar to the procedure outlined in Krühler et al. (2011b). The UVOT images were calibrated using the method described in Poole et al. (2008).

The photometric error was then estimated based on the contributions from photon statistics and goodness of the PSF fit (typically between 0.5 to $15 \%$ ), the absolute accuracy of the primary calibration system $(\approx 2-3 \%)$, the systematic scatter of different instrument/bandpasses with respect to the primary calibrators $(\approx 3-6 \%)$, or the uncertainty in the colour transformation (if applicable, $\approx 6-9 \%$ ).

The photometry described in the earlier paragraph inevitably contains a seeing-dependent fraction of the host light directly at the position of the transient. This contribution is best removed via differential imaging with deep reference frames from the same instrument/filter combination taken after the transient has faded completely. Given the vast number of different observers taking part in our photometry campaign, however, this procedure was not feasible in our case for all images. We instead used reference frames from a single telescope (Gemini-N, obtained $\sim 270$ days after the explosion) in three filters. We measure $g^{\prime}=24.62 \pm 0.10, r^{\prime}=24.09 \pm 0.09$, and $i^{\prime}=24.09 \pm 0.09 \mathrm{mag}$, which correspond to a host light contribution of $10 \%, 7 \%$, and $7 \%$ in $g^{\prime} r^{\prime} i^{\prime}$, respectively, at the maximum light of the $\mathrm{SN}$ at the position of the optical transient. To estimate the fraction in different filters, we scaled the above numbers to the respective filters using the spectral energy distribution (SED) of the host. We assume that this factor is similar for all data from various telescopes. We note that the values in Table A.1 are not corrected for this host contribution.

\section{The transient accompanying GRB 120422A}

Figure 2 displays the brightness evolution of the transient that accompanies GRB 120422A from the X-ray to the NIR bands. During the first three days, its brightness in the UVOT filters gradually decreases with a decay slope of $\alpha=0.2$ that is followed by a rebrightening, which peaked at $\sim 20$ after the GRB. The time scale and the colour evolution of the rebrightening are comparable to those of GRB-SNe (e.g. Zeh et al. 2004). The initially decaying transient could, therefore, be a superposition of the afterglow and the thermal emission of the cooling photosphere after the SN emerged. The key to understanding the evolution of the transient accompanying GRB 120422A is in determining how to disentangle the different radiation components. In the following sections, we present our results on each component.

\subsection{The stellar envelope cooling-phase}

Figure 3 displays SEDs at 0.054 and 0.267 days after the GRB. While afterglows have spectra formed by piecewise-connected power-laws from radio to X-rays (Sari et al. 1998), the cooling phase of the stellar envelope that was heated by the SN shock break-out is characterised by thermal emission peaking in the UV.

The early UV emission is indeed well fitted with a blackbody (for details, see Sect. 3.2.3). We measure a blackbody temperature of $k T_{\text {obs }} \sim 16 \mathrm{eV}(\approx 185000 \mathrm{~K})$ and a radius of $R_{\text {obs }} \sim 7 \times 10^{13} \mathrm{~cm}$ (both in the observer frame) at $T_{0}+0.054$ days. These values are consistent with the expectation from the shock break-out model (e.g. Ensman \& Burrows 1992; Campana et al. 2006, and references therein) and lie in the ballpark of the observed values of Ib/c SNe, such as 1993J (Richmond et al. 1994, 1996; Blinnikov et al. 1998), 1999ex (Stritzinger et al. 2002), 2008D (Soderberg et al. 2008; Malesani et al. 2009; Modjaz et al. 2009), and 2011dh (Arcavi et al. 2011; Soderberg et al. 2012; Ergon et al. 2014), and of the GRB-SNe, 2006aj (Campana et al. 2006) and 2010bh (Cano et al. 2011a; Olivares et al. 2012). In Sect. 3.2.3, we use the X-ray-to-NIR SED to provide further circumstantial evidence for the shock break-out interpretation.

The observed decline in the $u$ band between its first detection and $T_{0}+2.8$ days of $\sim 2 \mathrm{mag}$ is comparable to that observed in GRB 060218 (Campana et al. 2006). However, for this event these authors also reported an increase in brightness that lasted up to 0.57 days after the burst (shifted to the observer frame of GRB 120422A). This initial rise is not present in our data, although the first observation was at $86.4 \mathrm{~s}$ after the onset of the $\gamma$-ray emission.

\subsection{The afterglow emission}

\subsubsection{X-rays}

Zhang et al. (2012) reported that the early X-ray emission $(t<200 \mathrm{~s})$ is consistent with high-latitude emission from the prompt emission phase (e.g. Fenimore \& Sumner 1997; Kumar \& Panaitescu 2000; Dermer 2004), with evidence for small-scale deviation from power-law models (Starling et al. 2012), possibly due to a thermal component as seen in other GRBs (e.g. Campana et al. 2006; Page et al. 2011; Starling et al. 2011, 2012; Sparre \& Starling 2012; Friis \& Watson 2013). Friis \& Watson (2013) suggested that such a thermal component is not produced by the stellar photosphere but by the photosphere of the GRB jet. 


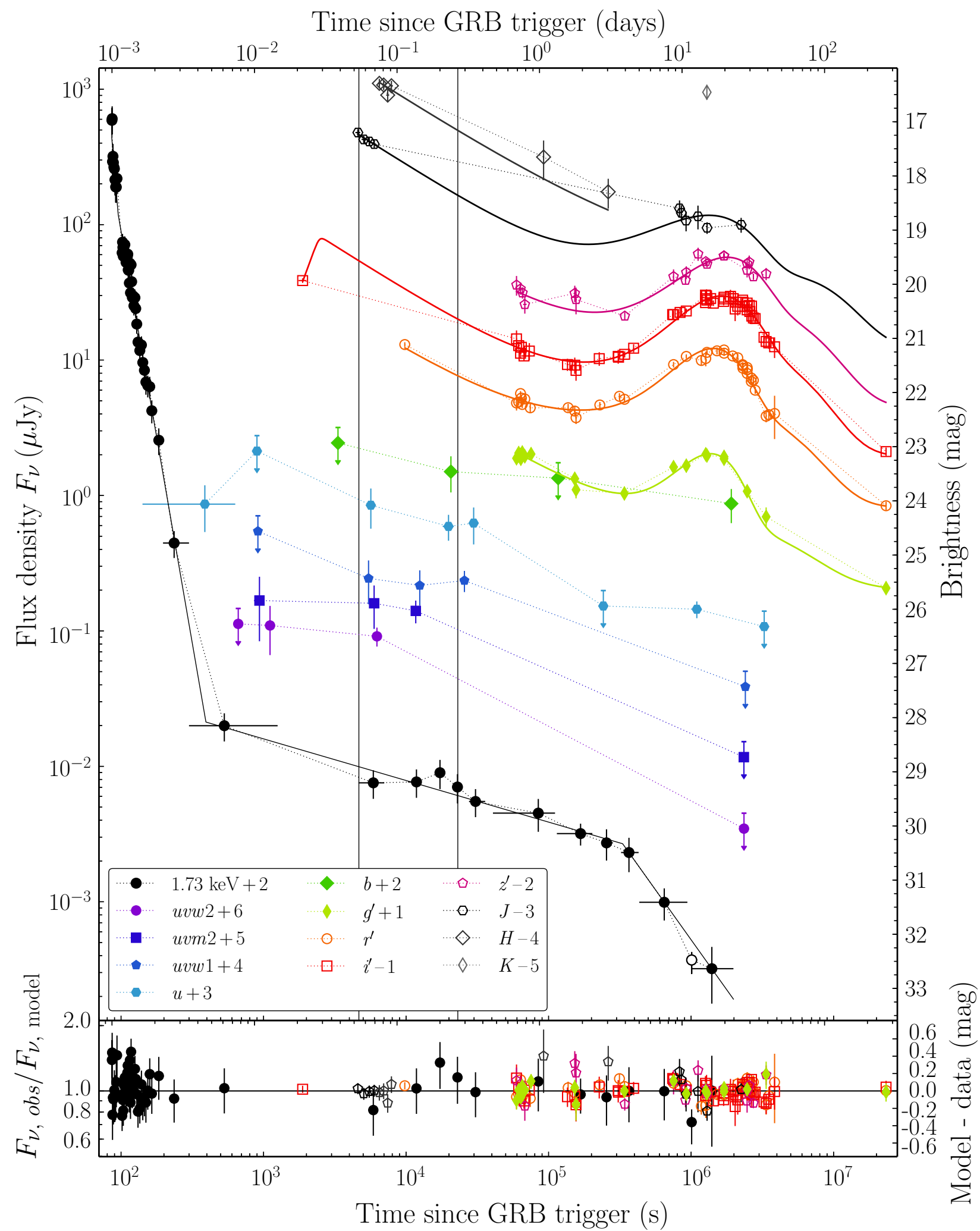

Fig. 2. X-ray, optical and NIR light curves of the transient that followed GRB 120422A. Arrows indicate $3 \sigma$ upper limits. The UVOT $v$-band upper limits are very shallow and not displayed. Data in the $g^{\prime} r^{\prime} i^{\prime} z^{\prime} J$ bands were modelled with a rescaled SN 1998bw template at $z=0.283$, which was superposed on a power-law (where the slope was identical in all bands) using the formalism in Zeh et al. (2004). The best-fit model parameters are shown in Table 3. Model light curves in bluer or redder filters are not shown, since they would require extrapolation of the spectral range of the SN1998bw template. Fit residuals are displayed in the bottom panel. The XMM-Newton observation was carried out at $980 \mathrm{ks}$ (open dot). The shifts (in magnitude) of the different bands are given in the legend. To convert the X-ray light curve to flux density, we assumed a spectral slope of $\beta=0.9$ and no spectral evolution (for details on the SED modelling see Sect. 3.2.3). Both assumptions have no implications on our analysis. The XMM-Newton data point was discarded from the light curve fit because of uncertainties in the cross-calibration between Swift/XRT and XMM-Newton. The vertical lines indicate the epochs of the X-ray-to-NIR SEDs presented in Sect. 3.2.3. Error bars can in some cases be smaller than the marker size. 
In the following, we focus on the emission at $>200 \mathrm{~s}$ after the burst.

At the time of our XMM-Newton observation, the X-ray spectrum is adequately fit as an absorbed power-law with a spectral slope of $\beta=0.94_{-0.11}^{+0.12}$ and absorption entirely consistent with the Galactic column $\left(3.71 \times 10^{20} \mathrm{~cm}^{-2}\right)$. The spectral slope is consistent with that derived from the late time XRT spectrum $(\beta=0.98 \pm 0.13)$ and suggests no late time spectral changes $(t>4600 \mathrm{~s})$. The spectral slope is typical for GRB afterglows at that phase.

The joint XRT and XMM-Newton light curve is shown in Fig. 2, where we converted the XRT observations to flux density based on the mean spectral index of the system (following Evans et al. 2009) and then added the XMM-Newton observations by assuming their measured spectral parameters. The X-ray light curve is adequately fit by a multiple broken power-law with indices of $\alpha_{1}=12.7 \pm 4.1, \alpha_{2}=6.09 \pm 0.16, \alpha_{3}=0.31 \pm 0.04$, and $\alpha_{4}=1.48 \pm 0.40$, and break times of $t_{b, 1}=95.3 \pm 3.2 \mathrm{~s}$, $t_{b, 2}=394 \pm 19 \mathrm{~s}$, and $t_{b, 3}=330.5 \pm 89.0 \mathrm{ks}$; the resulting $\chi^{2} /$ d.o.f. $=43.5 / 54$. We note that an early break is needed to fit the WT settling mode exposures, which has a chance improvement probability of $\sim 6.6 \times 10^{-5}$.

The steep to shallow to normal decay-phase evolution is typical for X-ray afterglows of high- $L$ GRBs (Nousek et al. 2006; Evans et al. 2010). In particular, the very rapid decay phase $\left(\propto t^{-13}\right)$ points to high-latitude emission and has not been observed for low- $L$ GRBs so far.

\subsubsection{Optical/NIR}

As mentioned before, the thermal emission of the cooling photosphere has an intrinsically blue spectrum and does not significantly contribute to the integrated emission in the optical and NIR. Therefore, the optical/NIR emission can be decomposed into three distinct emission components: i) the afterglow, which can be modelled with simple and broken power-law models; ii) the supernova; and iii) the host galaxy, which can be accounted for by a constant flux. To characterise the SN component, we follow the approach in Zeh et al. (2004). They used the multi-color light curves of the prototypical GRB-SN 1998bw (Galama et al. 1998; Patat et al. 2001) as templates. They derived the SN 1998bw light curves at both the given GRB redshift as well as the given observed band (including the cosmological $k$-correction) and then additionally modified the template with two parameters. The luminosity factor $k$ determines the SN peak luminosity in a given band in units of the SN 1998bw peak luminosity in that band. The stretch factor $s$ determines if the light curve evolution is faster $(s<1)$ or slower $(s>1)$ than that of SN 1998bw, whereby the actual evolutionary shape remains the same, and the explosion time is always identical to the GRB trigger time. However, we limit the SN modelling to the $g^{\prime} r^{\prime} i^{\prime} z^{\prime} J$ bands. Model light curves in bluer or redder filters require extrapolating the spectral range of the SN1998bw template.

The results of our fits are given in Table 3. In this section, we report on the properties of the afterglow, whereas those of the $\mathrm{SN}$ are given in Sect. 3.3.2. The light curve fits reveal that there is indeed a power-law component and, hence, provide strong evidence for an optical/NIR afterglow accompanying GRB 120422A. The fit with a simple power-law assumes that the afterglow light curve does not steepen until $T_{0}+270.2$ days, the time of the host galaxy observation. For a collimated outflow, the observer sees the edge of the jet at a certain time, which
Table 3. Properties of the SN modelling.

\begin{tabular}{|c|c|c|c|c|}
\hline \multicolumn{5}{|c|}{$\begin{array}{l}\text { Simple power-law }+ \text { free host magnitude } \\
\alpha_{1}=0.69 \pm 0.02\end{array}$} \\
\hline Band & $\begin{array}{l}\text { Host magnitude } \\
\text { (mag) }\end{array}$ & $\begin{array}{l}\text { Luminosity } \\
\text { factor } k\end{array}$ & $\begin{array}{l}\text { Stretch } \\
\text { factor } s\end{array}$ & $\chi^{2} /$ d.o.f. \\
\hline$g^{\prime}$ & $24.65 \pm 0.12$ & $0.86 \pm 0.03$ & $0.94 \pm 0.02$ & \\
\hline$r^{\prime}$ & $24.06 \pm 0.04$ & $1.25 \pm 0.02$ & $0.89 \pm 0.02$ & \\
\hline$i^{\prime}$ & $24.17 \pm 0.08$ & $1.10 \pm 0.01$ & $0.92 \pm 0.01$ & $194.9 / 146$ \\
\hline$z^{\prime}$ & $24.31 \pm 0.12$ & $0.99 \pm 0.02$ & $0.92 \pm 0.03$ & \\
\hline$J$ & $24.22 \pm 0.22$ & $1.12 \pm 0.09$ & $0.74 \pm 0.12$ & \\
\hline$H$ & $\ldots$ & $\ldots$ & $\ldots$ & \\
\hline \multicolumn{5}{|c|}{$\begin{array}{l}\text { Smoothly broken power-law }+ \text { fixed host magnitude } \\
\alpha_{1}=0.67 \pm 0.02, \alpha_{2}=2.00 \text { (fixed), } t_{b} \text { (days) }=9.7 \pm 4.4 \\
n=10 \text { (fixed) }\end{array}$} \\
\hline Band & $\begin{array}{l}\text { Host magnitude } \\
\text { (mag) }\end{array}$ & $\begin{array}{l}\text { Luminosity } \\
\text { factor } k\end{array}$ & $\begin{array}{l}\text { Stretch } \\
\text { factor } s\end{array}$ & $\chi^{2} /$ d.o.f. \\
\hline$g^{\prime}$ & 24.62 & $0.88 \pm 0.05$ & $0.97 \pm 0.02$ & \\
\hline$r^{\prime}$ & 24.09 & $1.25 \pm 0.02$ & $0.90 \pm 0.01$ & \\
\hline$i^{\prime}$ & 24.09 & $1.11 \pm 0.02$ & $0.92 \pm 0.01$ & $186.6 / 150$ \\
\hline$z^{\prime}$ & 24.15 & $0.99 \pm 0.03$ & $0.92 \pm 0.03$ & \\
\hline$J$ & 23.96 & $1.06 \pm 0.09$ & $0.68 \pm 0.09$ & \\
\hline$H$ & 23.84 & $\ldots$ & $\ldots$ & \\
\hline
\end{tabular}

Notes. Best-fit parameters of the $g^{\prime} r^{\prime} i^{\prime} z^{\prime} J H$ band light curve fits. We modelled $g^{\prime} r^{\prime} i^{\prime} z^{\prime} J$ light curves with a SN1998bw template redshifted to $z=0.2825$, as described in Zeh et al. (2004), which is superposed on a simple power-law or smoothly broken power-law (Beuermann et al. 1999), where $\alpha$ denotes the decay slope, $t_{\mathrm{b}}$ the break time, and $n$ the smoothness, to account for the early emission and the flux from the host galaxy at the explosion site. For the $H$ band, we used the afterglow models only. We assumed that the afterglow component evolves achromatically from the $g^{\prime}$ to the $H$ band. The supernova and afterglow light curve is equally well fitted with the two models. Column 2 gives the contribution of the host galaxy in the used aperture. See Sect. 3.3.2 for details.

results in a significant steepening (Sari et al. 1999). A jet break after 270 days has been observed in GRB 060729 (Grupe et al. 2010, see also Perley et al. 2014 for a further example of a very late jet break), but a typical value is $\sim 0.6$ day (rest-frame; e.g. Zeh et al. 2006; Racusin et al. 2009). We refitted the light curve with a smoothly broken power-law (Beuermann et al. 1999), where the post-break decay slope was fixed to 2 . The pre-break slope is identical to the value from the simple power-law fit. The jet-break time of $9.7 \pm 4.4$ days (observer frame) is still large and very uncertain, but its value is more consistent with the observed distribution in Racusin et al. (2009). A reason for this large uncertainty in the break time is the brightness of the SN.

Both afterglow models over-predict the $i^{\prime}$-band brightness at $T_{0}+1880 \mathrm{~s}$ by $0.9 \mathrm{mag}$. The required rise could be either due to the crossing of the injection frequency $v_{m}$ or due to the coasting phase before the afterglow blast wave began decelerating. In the former case, the slope of the rise $\alpha_{\mathrm{r}}$ is -0.5 (with $F_{v} \propto t^{-\alpha_{\mathrm{r}}}$; Sari et al. 1998), and in the latter, the slope is between -3 and -2 for constant-density medium and $>0.5$ for a free-stellar-wind density profile (Shen \& Matzner 2012).

The crossing of the injection frequency $v_{\mathrm{m}}$ is by definition a chromatic feature. It evolves $\propto t^{-3 / 2}$ (Sari et al. 1998). This means the ratio between break times in two different bands has to obey $t_{2} / t_{1}=\left(v_{2} / v_{1}\right)^{-2 / 3}$. The $J$ band has the earliest detection after the first $i^{\prime}$ observation and is not affected by the thermal emission from the cooling stellar photosphere. Since the $J$-band light curve is only decaying, $v_{\mathrm{m}}$ crossed this band at $t<4550 \mathrm{~s}$ after the burst and, hence, the $i^{\prime}$ band at $\$ 3260 \mathrm{~s}$. Already in 

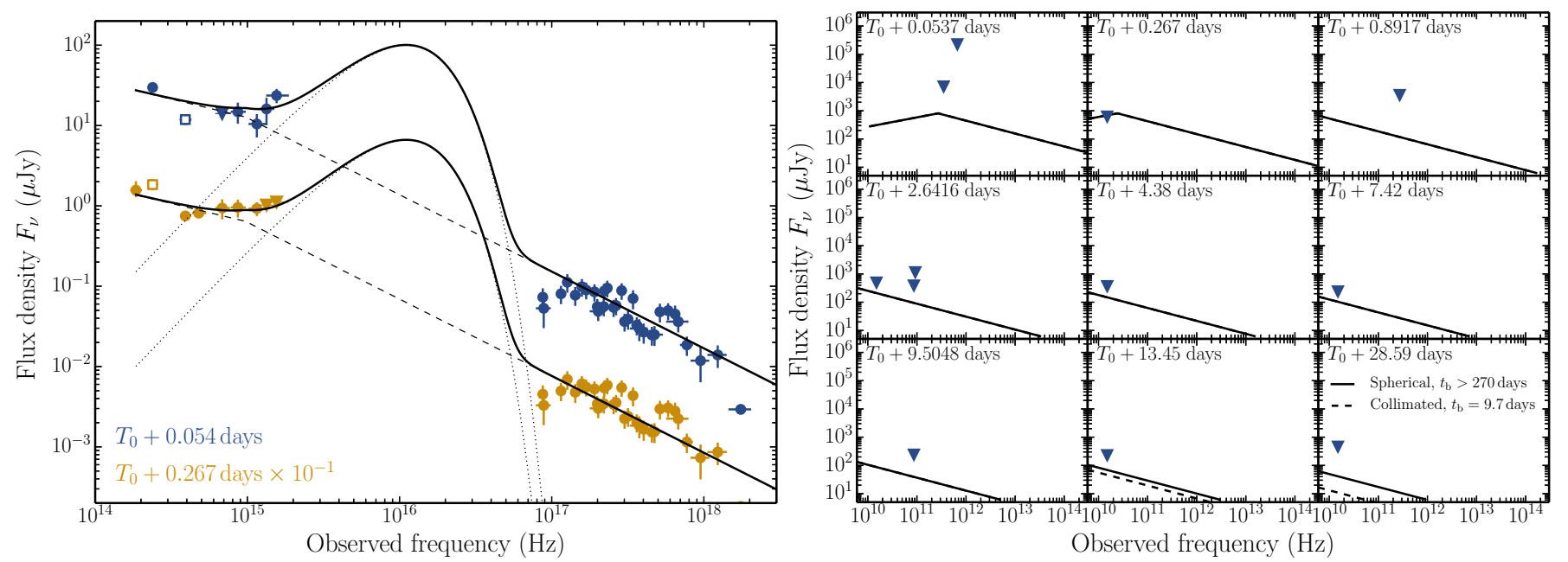

Fig. 3. Left: spectral energy distribution from the NIR to the X-ray at early epochs. The optical-to-X-ray SEDs are best described by the sum of an absorbed broken power-law (dashed lines) and a blackbody (dotted lines). Data excluded from the fits are shown as empty squares. If these data would be added, the fit statistic for an absorbed broken power-law (excluding the UV data) significantly worsens from $\chi^{2} /$ d.o.f. $=103.7 / 65$ to $\chi^{2} /$ d.o.f. $=159.1 / 67$. The interpolated values also significantly deviate from the best-fit model afterglow light curves (Fig. 2). Upper limits are shown by triangles. Right: extrapolation of the X-ray/NIR SED to the sub-mm-region compared to the available upper limits (Table 2). The NIR-to-X-ray SED from $T_{0}+0.267$ days was extrapolated to radio frequencies and evolved in time for a collimated and spherical expansion of the blast wave (for details see text). The AMI-LA measurement from $T_{0}+2.38$ was shifted to 2.6416 days, assuming the injection frequency to be blueward of the observed bandpass and using the scaling relations in Sari et al. (1998). This has no implications on our analysis.

the limiting case, the expected $i^{\prime}$-band magnitude is 0.24 mag brighter than the observed value. Considering the small photometric error of 0.04 mag makes the deviation statistically significant, and this scenario unlikely. The blast wave coasting into a free-stellar-wind density profile is also in conflict with our data, since we detect a clear rise and not a shallow decay.

A steep rise of $\alpha_{\mathrm{r}}=-2$ to -3 is fully consistent with our data. In both cases, the break time is $\sim 2500 \mathrm{~s}$ (observer frame). We hence identify the coasting phase into a constant-density circumburst medium as the most likely scenario ${ }^{12}$. Since the break time determines the transition from the coasting to the deceleration phase, it can be used to measure the initial Lorentz factor $\Gamma_{0}$ of the decelerating blast wave (Sari \& Piran 1999; Panaitescu \& Kumar 2000; Mészáros 2006). Following Molinari et al. (2007), we measure $\Gamma_{0} \sim 50$ using the observed break time and the measurement of the energy released during the prompt $\gamma$-ray emission, $E_{\text {iso }}=(1.6-3.2) \times 10^{50} \mathrm{erg}$.

\subsubsection{The SED from the radio to the X-rays}

To characterise the afterglow properties in more detail, we model the NIR-to-X-ray SED. We limit this analysis to $<T_{0}+0.6$ day, since SN 2012bz started contributing a non-negligible amount of flux to the integrated light at later times. We choose the epochs $T_{0}+0.054$ days and $T_{0}+0.267$ days to match the dates of the sub-mm observations. The optical and NIR fluxes were

\footnotetext{
12 Applying the closure relations between spectral and temporal slopes (Sari et al. 1998; Chevalier \& Li 2000) to the NIR/optical afterglow and using the spectral slope of $\beta_{0} \sim 0.46$ derived in Sect. 3.2.3, we find that the relation for a blast wave traversing a constant density medium $\left(\alpha_{\text {exp }}=3 \beta / 2=0.7, \alpha_{\text {obs }}=0.69\right)$ is satisified, if $v_{\mathrm{m}}<v<v_{\mathrm{c}}$ and the blast wave is expanding spherically. A free stellar-wind-density profile, as proposed by Zhang et al. (2012), does not fulfil the closure relations $\left(\alpha_{\exp }=3 \beta / 2+0.5=1.2\right)$, which provides circumstantial evidence for our interpretation of the early rise in the $i^{\prime}$ band.
}

obtained through interpolation between adjacent data points ${ }^{13}$. Errors were estimated by interpolation. The flux scales of the XRT and XMM (MOS1, MOS2, and PN) data were adjusted to the brightness of the X-ray afterglow at the respective epochs.

These early SEDs may in principle contain evidence for the thermal emission from the cooling photosphere after the shock break-out (Sect. 3.1). The SEDs of GRB afterglows are well described through single or broken power-laws, which are possibly altered by dust and metal absorption. A single (absorbed) power law almost always suffices to fit the optical/NIR data. In the case of GRB 120422A, the simultaneous fit of the NIR-toUV data with a single power law is very poor $\left(\chi^{2}=16.6\right.$ for 8 d.o.f.). At both epochs, a clear excess is apparent in the UV data. Unaccounted extinction would only make the intrinsic SED even bluer ${ }^{14}$.

To isolate this radiation component, we fit the two NIR-toX-ray SEDs by excluding the UV data with absorbed power-law and broken power-law models. The SEDs are best described by an absorbed broken power-law with $\beta_{\mathrm{x}} \sim 0.97$ and $\beta_{\mathrm{o}} \sim \beta_{\mathrm{x}}-0.5$, as expected for the simplest blast-wave model, where the cooling break in the synchrotron spectrum is between the optical and the $\mathrm{X}$-rays and has a break energy of the order of eV (Fig. 3). The degeneracy between the spectral slope and the break energy is very strong (Fig. C.1). A 7\% larger spectral slope would double the break energy. In comparison, the cooling frequency $\left(v_{\mathrm{c}} \propto t^{-1 / 2}\right)$ would decrease by a factor of 2.2 between both epochs, which is within the uncertainty of the cooling frequency measurement. Without loss of generality, we assume a break energy of $4 \mathrm{eV}$ in the following (Fig. 3).

In the next step, we add the UV data to elucidate the nature of the UV excess. By adding these data, the fit statistics for the

${ }^{13}$ In the UV, there are cases where one of the adjacent data points is an upper limit but the epoch of the SED is very close to the time of the detection $(\Delta t<0.1 \mathrm{dex})$. In these cases, we treated the interpolated data point as a detection but not as an upper limit.

${ }^{14}$ In Sect. 4.1, we show that the line-of-sight extinction is negligible. 
broken power-law model worsen from $\chi^{2}=103.7$ for 65 d.o.f. to $\chi^{2}=120.8$ for 72 d.o.f. Although the chance probability of $17 \%$ is statistically not significant, we note that the residuals of the UV data increase with decreasing wavelength (Fig. 3). Fitting the SED from 2000 to $2600 \AA$ at $T_{0}+0.054$ days with a simple power law $\left(F_{v} \propto v^{-\beta}\right)$ returns a spectral slope of $\beta=-3.3 \pm 1.6$. Such a hard spectrum is inconsistent with any afterglow model (see e.g. Zhang \& Mészáros 2004) but is fully consistent with the slope or the Rayleigh-Jeans tail of a blackbody spectrum $\left(F_{v} \propto v^{2} T\right)$.

Measured temperatures of the cooling photospheres after the shock break-out of GRBs 060218 and 100316D were between several $10 \mathrm{eV}$ and a few $100 \mathrm{eV}$ (Campana et al. 2006; Olivares et al. 2012). The soft X-ray bands of the XMM-Newton and Swift/XRT spectra show no evidence for a prominent thermal component at the given epochs, which limits the temperature $k_{\mathrm{B}} T$ to several $10 \mathrm{eV}$. Considering that the $w 2$ band, the bluest UV filter in our campaign, is just sensitive to emission at $\sim 6 \mathrm{eV}$, our data can only probe the Rayleigh-Jeans tail, which naturally explains why the excess is not stronger.

To constrain this thermal component, we add a blackbody to the power-law model that is defined by

$$
B B(E ; C, T)=1.0344 \times 10^{-3} C \frac{E^{2} \Delta E}{\exp \left(E / k_{\mathrm{B}} T\right)-1},
$$

where the numerical constant $C$ is defined as $R_{\mathrm{km}}^{2} / D_{10 \mathrm{kpc}}^{2}, R_{\mathrm{km}}$ is the blackbody radius in $\mathrm{km}, D_{10 \mathrm{pc}}$ is the distance in units of $10 \mathrm{kpc}, k_{\mathrm{B}}$ the Boltzmann constant, $T$ the temperature in units of $\mathrm{keV}$, and $E$ the energy and $\Delta E$ is the width of the energy bin, where both are in units of $\mathrm{keV}$. Given the sparsity of UV data at $T_{0}+0.267$ days, the normalisation constant and the temperature of the blackbody component cannot be constrained simultaneously. We therefore assume the blackbody temperature to not evolve.

The fit to both SEDs is shown in Fig. 3. The best fit is characterised by a spectral slope of $\beta_{\mathrm{o}} \sim 0.46$ (unchanged with respect to the fit without UV data), a blackbody temperature of $k_{\mathrm{B}} T_{\mathrm{obs}} \sim 16 \mathrm{eV}\left(T_{\mathrm{obs}} \approx 185000 \mathrm{~K}\right)$, and a blackbody radius of $R_{\text {obs }} \sim 7 \times 10^{13} \mathrm{~cm}$ at $1.4 \mathrm{hr}$ after the burst. The blackbody component in the second epoch is barely constrained because of the limited amount of UV data. The fit statistics are $\chi^{2} /$ d.o.f. $=112.4 / 69$. We note that the fit statistics are affected by scatter in the X-ray spectra.

The peak of an afterglow spectrum is typically at $\mathrm{cm} / \mathrm{sub}-\mathrm{mm}$ wavelengths and usually crosses this band within the first week. We therefore extrapolate the afterglow SED from $T_{0}+0.267$ days to radio wavelengths (Fig. 3) and evolve the SED to all epochs of the radio and sub-mm observation listed in Table 2. We used the scaling relations for the injection frequency and the peak flux density for a spherical expansion and a post-jet peak evolution respectively from Sari et al. (1998, 1999). In both dynamical scenarios, the peak flux density is $\lesssim 810 \mu \mathrm{Jy}$, which corresponds to a specific luminosity of $\lesssim 2 \times 10^{30} \mathrm{erg} \mathrm{s}^{-1} \mathrm{~Hz}^{-1}$ before the jet break occurred.

\subsection{Supernova properties}

\subsubsection{Supernova spectrum}

Our spectra of SN 2012bz are displayed in Fig. 4. The very early spectra are dominated by a smooth power-law continuum, which is characteristic of GRB afterglows. At around 4.7 days, after the transient started re-brightening (Fig. 2), the shape of the spectrum changed, and became redder. By May 1 (8.8 days after the GRB), the spectrum clearly started resembling that of a supernova with broad lines (Sect. 5.1.1; Malesani et al. 2012a; Sánchez-Ramírez et al. 2012; Wiersema et al. 2012). By May 10 (18.7 days after the GRB), the transformation was complete, and our X-shooter spectra from +18.7 to +24.7 days were found to be very similar to those of other broad-lined Type Ic SNe accompanying GRBs (Fig. 10). The Magellan spectrum obtained 26.8 days after the GRB has a low $\mathrm{S} / \mathrm{N}$, despite showing absorption troughs at locations consistent with the previous data, and should be interpreted with great caution. The modelling of the spectral evolution will be presented in a forthcoming paper.

Usually, GRB-SN expansion velocities are reported for the Si II $\lambda 6355$ feature, while the Ca II NIR triplet at $8600 \AA$ is reported sometimes as the only alternative (Patat et al. 2001; Hjorth et al. 2003; Chornock et al. 2010; Bufano et al. 2012). In the case of SN 2012bz, the Si II line is contaminated by the telluric A-band, while the Ca IR triplet is redshifted outside the optical spectrum. For this reason, we chose to measure the expansion velocities based on the Fe II $\lambda 5169$ feature. In addition, this feature appears earlier than the Si II feature and its minimum is easier to locate, as it lies between two clearly visible maxima (Figs. 4, 10). This makes it a potentially better expansion velocity tracer for GRB-SNe than Si II, which is super-imposed on a blue continuum, and it is not always easy to locate and measure, especially at early times.

We have used the fiducial rest-wavelength of $5169 \AA$ for Fe II, as done e.g. in Hamuy \& Pinto (2002) for the expansion velocities of Type IIP SNe. If this identification is not correct for GRB-SNe due to blending, we stress that even these measurements are still valuable to monitor the expansion velocity evolution and for comparison between different objects, as long as the measurements are done consistently. Based on these assumptions, we present the first, to our knowledge, diagram of GRB-SNe expansion velocities based on Fe II $\lambda 5169$ (Fig. 5). The velocities (of the order of 5000-50000 $\mathrm{km} \mathrm{s}^{-1}$ ) are in the range measured for other SNe associated with GRBs. SN 2010bh shows the fastest explosion velocities as seen from $\mathrm{Si}$ II, while SN 2006aj the slowest (Chornock et al. 2010; Bufano et al. 2012). SN 2012bz shows large velocities at three days past explosion (The earliest spectrum where a measurement is possible.) and slows down to $17000 \mathrm{~km} \mathrm{~s}^{-1} \sim 21$ days later. This behaviour is very similar to $\mathrm{SN} 2003 \mathrm{dh}$, which is associated with the high- $L$ GRB 030329 (Hjorth et al. 2003).

\subsubsection{Absolute magnitude}

The luminosities of SNe are usually reported in the rest-frame $V$ band. The $r^{\prime}$ bandpass (observer frame) partly overlaps with the rest-frame $V$ band, though it is not identical. We compute the $k$-corrected $V$-band magnitude from the $r^{\prime}$-band maximum, following Hogg et al. (2002) and using the X-shooter spectrum from $T_{0}+18.7$ days (i.e. $<2$ days after the maximum in $r^{\prime}$ band) as a weighing function. The peak luminosity of $M_{V}=-19.7 \mathrm{mag}$ is 0.3 mag brighter than SN 1998bw, if we use the face value of $M_{V}=-19.4$ mag from Cano et al. (2011b).

Measuring the SN luminosity by using a $k$-correction from the observed spectrum is the most direct and accurate approach. However, the number of spectroscopically confirmed GRB-SNe is still small. Moreover, optical spectroscopy is limited to mostly low redshifts $(z<0.3)$ because of the prohibitively long exposures required for a $M_{V} \sim-19$ mag SN at higher redshifts. In addition, the useful wavelength range is reduced to the red part of 


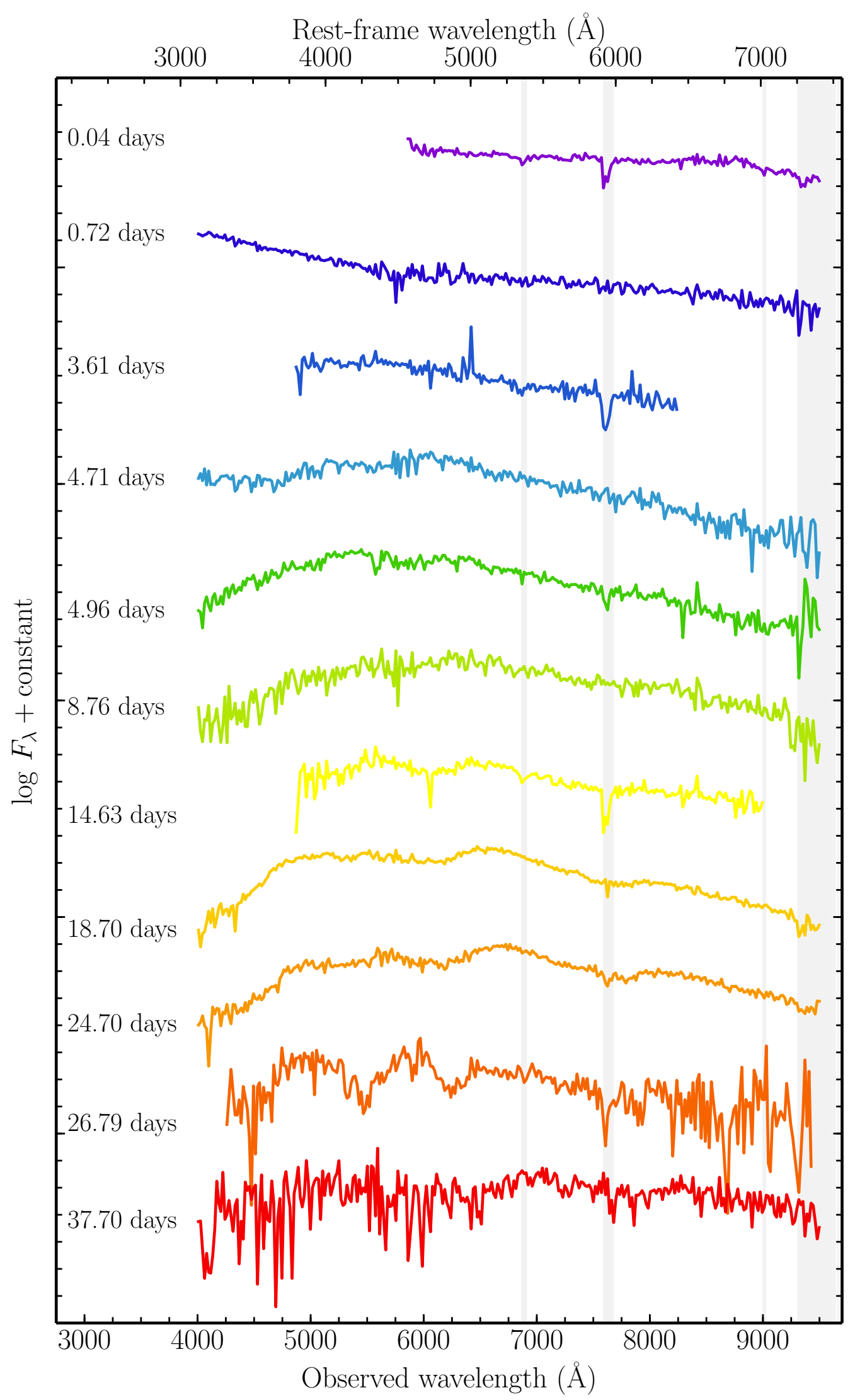

Fig. 4. Spectral evolution of the optical transient accompanying GRB 120422A. The first two epochs show a smooth power-law-shaped continuum, which is characteristic of GRB afterglows. After the transient started re-brightening, the shape of the spectrum becomes redder. At 8.8 days after the GRB, the spectrum has clearly started to resemble that of a broad-lined SN. At 18.7 days, the transformation was complete, and the spectra look similar to other GRB-SNe. All spectra were shifted vertically by an arbitrary constant. They were rebinned (18 $\AA$ ) to increase $\mathrm{S} / \mathrm{N}$ for presentation purposes. We only display spectra with a large spectral range. Strong telluric lines (transparency $<20 \%$ ) are highlighted by the grey-shaded areas. 


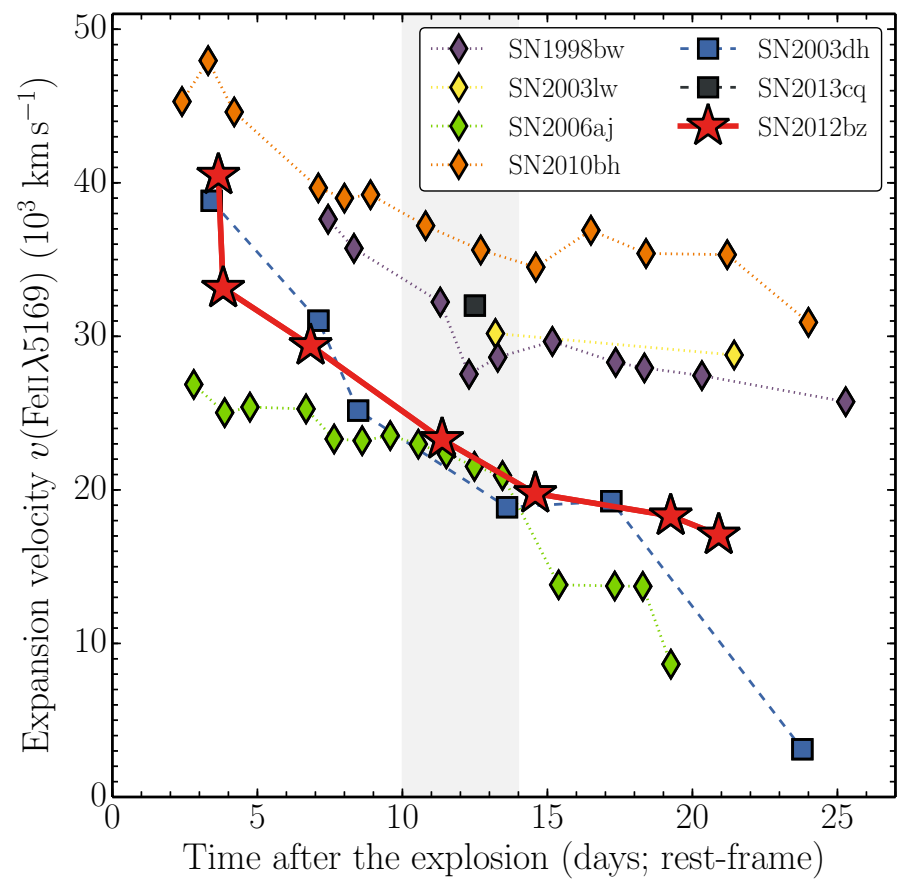

Fig. 5. Evolution of the expansion velocities measured from Fe II $\lambda 5169$ for SN 2012bz and six GRB-SNe of low (diamonds) and highluminosity GRBs (boxes) with good spectroscopic data. Measurements were performed on our data as well as on the spectra of Patat et al. (2001), Hjorth et al. (2003), Malesani et al. (2004), Pian et al. (2006), and Bufano et al. (2012). The value of SN 2013cq was taken from Xu et al. (2013). The grey-shaded area displays the interval of observed GRB-SN peak times.

the observed spectrum due to line blanketing by iron, as the restframe UV moves into the optical $V$ band (Filippenko 1997). An alternative approach is to look for "late red bumps" in afterglow light curves, which are due to the SNe. The best-fit parameters of the SN bump with SN 1998bw templates in the $g^{\prime} r^{\prime} i^{\prime} z^{\prime} J$ bands, as shown in Sect. 3.2.2, are displayed in Table 3. The fit reveals that SN 2012bz is 0.3 mag more luminous than SN 1998bw in the observed $r^{\prime}$ band. The evolution is slightly faster than that of SN 1998bw, and it is somewhat redder.

\subsubsection{The explosion-physics parameters}

The peak and width of an SN light curve are determined by the explosion-physics parameters, such as ejecta mass $M_{\mathrm{ej}}$, ${ }^{56} \mathrm{Ni}$ mass $M_{\mathrm{Ni}}$, and kinetic energy $E_{\mathrm{k}}$ of the $\mathrm{SN}$ ejecta. These values are estimated from the bolometric light curve. An estimate of the bolometric light curve was constructed using $g^{\prime} r^{\prime} i^{\prime} z^{\prime}$ photometric points, as coverage outside these bands is limited around the SN peak. The light curves in each filter were fitted with spline interpolations starting at two days past the GRB trigger, such that an estimated magnitude for all four bands was available at each epoch of observation. Magnitudes were converted into monochromatic fluxes at the effective (rest-frame) wavelengths of the filters for every epoch to produce an $\mathrm{SED}^{15}$. Each SED was then integrated over the limits of the filter wavelength range, which is the blue edge of $g^{\prime}$ and the red edge of $z^{\prime}(\sim 3000-8000 \AA)$. The SED was tied to zero flux at these

\footnotetext{
${ }^{15}$ Since we are evaluating the SED for every observation, nearby epochs (within $<0.2$ day of each other) were first calculated individually and then averaged when producing the final light curve for clarity.
}

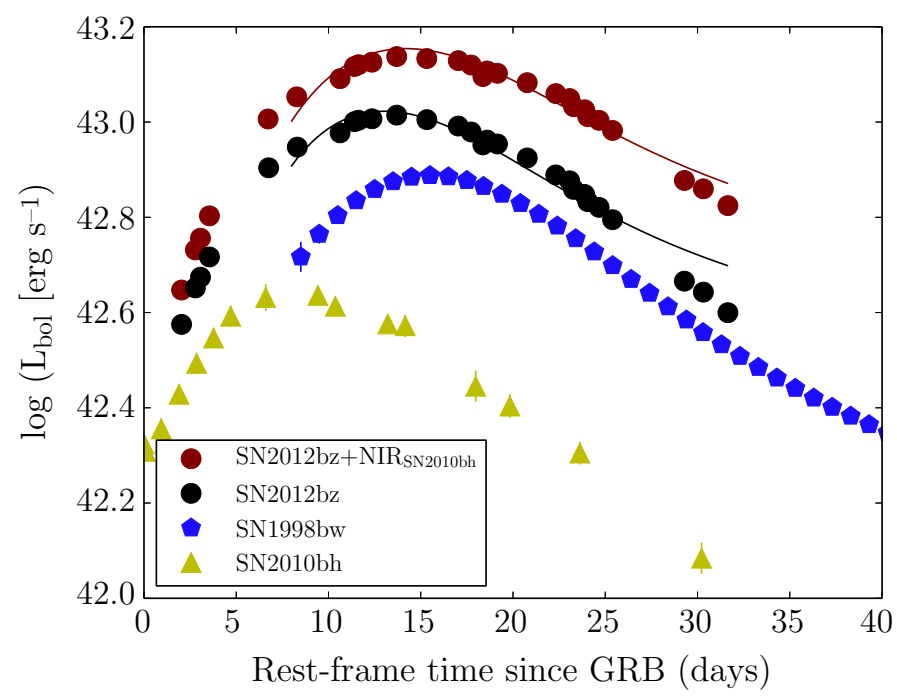

Fig. 6. Pseudo-bolometric light curves of SN 2012bz from direct integration of the SED over $g^{\prime} r^{\prime} i^{\prime} z^{\prime}$ filters, and after including a NIR contribution as found for SN 2010bh. For comparison, the UBVRI light curve of SN 1998bw (Clocchiatti et al. 2011) and the $g^{\prime} r^{\prime} i^{\prime} z^{\prime} J H$ light curve of SN 2010bh are shown (Olivares et al. 2012). The models for SN 2012bz are shown as solid lines. Early light-curve time data are not fitted as the analytical model does not account for other non-negligible sources of luminosity at these times (Sect. 3.3.3). Only photometric and calibration uncertainties are included in the error bars, which are usually smaller than the size of the plot symbol.

limits, which were defined as the wavelength at which the respective filter's normalised transmission curve falls below $10 \%$. The integrated fluxes were converted to luminosities using the redshift and cosmology adopted previously. The resulting light curve (Fig. 6) gives a luminosity of the SN over approximately the optical wavelength range.

Contributions to the flux outside this regime, however, are not insignificant with the optical accounting for $\sim 50-60 \%$ of the bolometric flux for stripped-envelope SNe (Lyman et al. 2014). Of particular importance is the contribution from the NIR, wherein the fraction of the total luminosity emitted increases with time and reaches a comparable contribution to the optical within 30 days (e.g. Valenti et al. 2008; Cano et al. 2011a). We estimate this missing NIR flux by using the fractional NIR flux of a similar event, as done in Cano et al. (2011a). A photometric study by Olivares et al. (2012) of the low redshift $(z=0.059)$ XRF 100316D/SN 2010bh contains well-sampled light curves in the $z^{\prime} J H$ bands, extending upon our rest-frame wavelength limits. The contribution of wavelengths $>8000 \AA$ to the flux was determined by first integrating SN 2010bh's de-reddened SED over the same wavelength range used for SN $2012 \mathrm{bz}$ above and then over the wavelength range redward of $8000 \AA$. Thus, for each epoch of observation, we obtain the NIR contribution as a fraction of the optical flux. The phase of the contributions were normalised, so $t=0$ was the peak of the respective $\mathrm{SNe}$ and stretched by a factor $\Delta m_{15,(3000-8000)} \AA$ to match the light curve shape of the two SNe $\left(\Delta m_{15,(3000-8000)} \AA=0.78\right.$ for SN $2012 \mathrm{bz}$, 1.00 for SN 2010bh) ${ }^{16}$. The fractional values were interpolated using a smooth spline to sample it at the epochs of observations of SN 2012bz, and the appropriate amount was added to the optical flux. This gives a NIR-corrected light curve covering

\footnotetext{
16 Phillips (1993) introduced $\Delta m_{15}$ as the decline in the brightness between the maximum and 15 days post maximum in $B$ band.
} 
3000-17000 A. No attempt was made to account for flux missed below $3000 \AA$ due to the paucity of data that constrained the UV in such objects. However, contributions from the UV account for only $\sim 5-15 \%$ of the bolometric flux around peak (Lyman et al. 2014).

The bolometric light curve was modelled using the simplified analytical prescription of Arnett (1982), which were updated by Valenti et al. (2008), to obtain estimates of $M_{\mathrm{Ni}}, M_{\mathrm{ej}}$ and $E_{\mathrm{k}}$. Since obtaining a truly bolometric light curve is unfeasible, we use our optical and optical+NIR correction light curves as approximations. Our data cover the photospheric phase of SN evolution, when the ejecta are optically thick. The opacity is chosen to be $\kappa=0.07 \mathrm{~cm}^{2} \mathrm{~g}^{-1}$ (see Cano et al. 2011a). To constrain the $E_{\mathrm{k}} / M_{\mathrm{ej}}$ ratio, a scale velocity is required (see Eq. (54) in Arnett 1982). This is taken to be the photospheric velocity $\left(v_{\mathrm{ph}}\right)$ at peak. The Fe II lines are a good tracer of $v_{\text {ph }}$ (Valenti et al. 2011), and the peak of the pseudo-bolometric light curve occurs at $\sim 13.9$ days (from fitting low-order polynomials around peak). Using data in Fig. 5, we take $20500 \mathrm{~km} \mathrm{~s}^{-1}$ as an estimate of $v_{\text {ph }}$ at peak by linearly interpolating between the measurements taken from spectra at epochs of 11.380 days and 14.575 days.

Fitting the optical bolometric light curve reveals the following parameters: $M_{\mathrm{Ni}}=0.40 \pm 0.01 M_{\odot}, M_{\mathrm{ej}}=4.72 \pm 0.04 M_{\odot}$, and $E_{\mathrm{k}}=(3.29 \pm 0.03) \times 10^{52} \mathrm{erg}$. When the NIR contribution is included from SN 2010bh, we obtain $M_{\mathrm{Ni}}=0.58 \pm 0.01 M_{\odot}$, $M_{\mathrm{ej}}=5.87 \pm 0.03 M_{\odot}$, and $E_{\mathrm{k}}=(4.10 \pm 0.03) \times 10^{52} \mathrm{erg}$. The first eight days were ignored in the fit as contributions from other sources (GRB afterglow and cooling phase following the shock break-out) would compromise the assumptions of the SN model.

It is crucial to note that the errors quoted here include only the statistical uncertainties relating to the construction of the pseudo-bolometric light curves. Systematic errors arise from both the simplifying assumptions in the model (spherical symmetry, centrally concentrated ${ }^{56} \mathrm{Ni}$ mass etc.) and our choice of parameters for the fit, which typically dominate the statistical errors. For example, taking an uncertainty in $v_{\mathrm{ph}}$ of $2000 \mathrm{~km} \mathrm{~s}^{-1}$ translates into an error in $M_{\mathrm{ej}}$ and $E_{\mathrm{k}}$ of $\sim 10 \%$ and $\sim 25 \%$, respectively. The two-component model for very energetic supernovae $\left(E_{\mathrm{k}} \gtrsim 5 \times 10^{51} \mathrm{erg}\right.$ ) by Maeda et al. (2003) would also suggest that we are only observing the outer, lower density region of the ejecta during the photospheric phase ( $\$ 30$ days), and a fraction is hidden in a denser, inner component during this time. Although the afterglow component is not expected to contribute significantly around the SN peak, given that different afterglow models do not significantly affect the $k, s$ parameters (Sect. 3.3.2), potential contamination by underlying host galaxy light is included in this bolometric light curve (Sect. 2.3).

Melandri et al. (2012) modelled SN 2012bz using a scaled spectral model for SN $2003 \mathrm{dh}$ to obtain estimates of the physical parameters. They obtained values of $M_{\mathrm{Ni}} \approx 0.35 M_{\odot}, M_{\mathrm{ej}} \approx$ $7 M_{\odot}$, and $E_{\mathrm{k}} \approx 3.5 \times 10^{52}$ erg using a bolometric light curve covering 3300-7400 $\AA$. Comparing these to our values for the optical (3000-8000 $\AA$ ) bolometric light curve, the $M_{\mathrm{Ni}}$ values are in good agreement. Given our slightly extended wavelength range, the $E_{\mathrm{k}}$ values are consistent; however, their derived ejected mass is larger than our measurement. Differences could be caused by the choice of photospheric velocity $v_{\mathrm{ph}}$, asymmetries, or varying opacity $\kappa$, which spectral modelling can account for.

\section{Environments}

Absorption and emission lines are powerful diagnostics to characterise the gas- and dust-phase properties of interstellar media,
Table 4. Absorption and emission lines at the explosion and the host site.

\begin{tabular}{|c|c|c|c|c|}
\hline$\lambda_{\text {obs }}(\AA)$ & Transition & Redshift & $E W_{\text {obs }}(\AA)$ & $\begin{array}{c}F \times 10^{16} \\
\left(\mathrm{erg} \mathrm{cm}^{-2} \mathrm{~s}^{-1}\right)\end{array}$ \\
\hline \multicolumn{5}{|c|}{ Explosion site $\left(\langle z\rangle_{\mathrm{abs}}=0.28253,\langle z\rangle_{\mathrm{em}}=0.28259\right)$} \\
\hline 3586.22 & Mg II $\lambda 2796$ & 0.2824 & $3.25 \pm 0.42$ & \\
\hline 3595.95 & $\operatorname{Mg}$ II $\lambda 2803$ & 0.2827 & $1.86 \pm 0.46$ & \\
\hline & $\operatorname{Mg} I \lambda 2852$ & & $<1.57$ & \\
\hline 4779.79 & {$[\mathrm{O}$ II $] \lambda 3727$} & 0.28245 & $\ldots$ & $0.09 \pm 0.01$ \\
\hline 4783.37 & [O II] $\lambda 3729$ & 0.28245 & & $0.16 \pm 0.01$ \\
\hline 5046.00 & Ca II $\lambda 3934$ & 0.2825 & & $0.75 \pm 0.25$ \\
\hline & Ca II $\lambda 3968$ & $\ldots$ & $<1.08$ & \\
\hline 6236.87 & $\mathrm{H} \beta$ & & $\ldots$ & $0.05 \pm 0.04$ \\
\hline 6362.18 & [O III] $\lambda 4959$ & 0.28262 & & $0.05 \pm 0.02$ \\
\hline 6423.34 & {$[\mathrm{O} \mathrm{III]} \lambda 5007$} & 0.28255 & & $0.19 \pm 0.02$ \\
\hline 8419.55 & $\mathrm{H} \alpha$ & 0.28257 & & $0.24 \pm 0.01$ \\
\hline 8447.92 & {$[\mathrm{~N}$ II $] \lambda 6583$} & 0.28286 & $\ldots$ & $0.06 \pm 0.02$ \\
\hline 8616.96 & {$[\mathrm{~S}$ II] $\lambda 6717$} & 0.28261 & $\ldots$ & $0.03 \pm 0.01$ \\
\hline \multicolumn{5}{|c|}{ Host site $\left(\mathrm{PA}=41^{\circ},\langle z\rangle_{\mathrm{em}}=0.28256\right)$} \\
\hline 4780.16 & {$[\mathrm{O}$ II] $\lambda 3727$} & 0.28254 & & $2.30 \pm 0.03$ \\
\hline 4783.73 & [O II] $\lambda 3729$ & 0.28254 & .. & $3.50 \pm 0.67$ \\
\hline 4920.46 & $\mathrm{H} \eta$ & 0.28255 & $\ldots$ & $0.09 \pm 0.01$ \\
\hline 4963.37 & {$[\mathrm{Ne}$ III] $\lambda 3869$} & 0.28262 & $\ldots$ & $0.27 \pm 0.02$ \\
\hline 4979.74 & $\mathrm{H} \zeta$ & 0.28248 & $\ldots$ & $0.20 \pm 0.01$ \\
\hline 5093.04 & $\mathrm{H} \epsilon$ & 0.28250 & $\ldots$ & $0.19 \pm 0.02$ \\
\hline 5262.10 & $\mathrm{H} \delta$ & 0.28252 & $\ldots$ & $0.30 \pm 0.02$ \\
\hline 5567.86 & $\mathrm{H} \gamma^{a}$ & 0.28242 & & $0.59 \pm 0.04$ \\
\hline 6236.89 & $\mathrm{H} \beta$ & 0.28260 & & $1.28 \pm 0.04$ \\
\hline 6362.15 & [O III] $\lambda 4959$ & 0.28261 & $\cdots$ & $0.83 \pm 0.03$ \\
\hline 6423.60 & [O III] $\lambda 5007$ & 0.28261 & $\ldots$ & $2.51 \pm 0.05$ \\
\hline 8419.75 & $\mathrm{H} \alpha^{b}$ & 0.28260 & $\ldots$ & $5.36 \pm 0.05$ \\
\hline 8446.38 & {$[\mathrm{~N}$ II] $] \lambda 6583$} & 0.28262 & $\ldots$ & $0.81 \pm 0.04$ \\
\hline 8616.90 & [S II] $\lambda 6717$ & 0.28260 & $\ldots$ & $0.91 \pm 0.02$ \\
\hline 8635.40 & {$[\mathrm{~S}$ II] $\lambda 6731$} & 0.28260 & $\ldots$ & $0.67 \pm 0.03$ \\
\hline
\end{tabular}

Notes. The reported wavelengths were derived from the first momentum of a line profile (see Figs. E.1 and E.2). The fluxes were corrected for foreground extinction. For each spectrum, we report the average absorption- $\left(\langle z\rangle_{\mathrm{abs}}\right)$ and emission-line $\left(\langle z\rangle_{\mathrm{em}}\right)$ redshift if available. (a) Blended line. (b) This value is the total flux of both velocity components.

such as the extinction, metallicity, and star-formation rate (SFR). Since long GRBs are associated with massive stars, these diagnostics give the unique opportunity to study star-forming regions in distant galaxies. In the following, we present our findings on the explosion site, the host galaxy, and the large scale environment (For an independent analysis, see Levesque et al. 2012).

\subsection{The explosion site}

The X-shooter spectrum, which was obtained on 23 April (17.2 h post burst; see Fig. E.1), exhibits two absorption lines, which we identify as Mg II $\lambda \lambda 2796,2803$ (see Table 4). After applying the heliocentric correction, we measure a mean absorption-line redshift of $z_{\mathrm{abs}}=0.28253 \pm 0.00008$ (the error denotes the standard error of the mean), which refines the redshift measurements of Schulze et al. (2012b) and Tanvir et al. (2012).

Both lines lie in a rather noisy part of the spectrum. To measure their equivalent widths, we rebinned the spectrum by a factor of two to increase the $\mathrm{S} / \mathrm{N}$ (i.e. a wavelength binning of $0.4 \AA$ ) and fixed the aperture size for the weaker $\mathrm{Mg}$ II line to $100 \mathrm{~km} \mathrm{~s}^{-1}$ (the FWHM of the Mg II $\lambda 2796$ absorption 
line). Their rest-frame EWs are listed in Table 4. The observed line ratio of $1.7 \pm 0.5$ is not well constrained. It is consistent with the theoretical expected line ratio for an optically thin line but also for a saturated line. Assuming the weak line regime, we can place a lower limit of $\log N \geq 13.8$ on the $\mathrm{Mg}$ II column density. When Mg II is detected, three further absorption lines are usually detected at longer wavelengths as well: Mg I $\lambda 2852$ and Ca II $\lambda \lambda 3934,3969$. Only Ca II $\lambda 3934$ is detected at $\lesssim 3 \sigma$ c.l. To place limits on their rest-frame EWs, we measure the noise within an aperture of $2 \times F W H M(\mathrm{Mg}$ II $\lambda 2796)$ at the wavelength of each line. Table 4 displays their derived upper limits. We caution that Mg II absorption lines can be broader than other absorption lines; hence, our upper limits might not be very stringent.

We also detect several emission lines that are summarised in Table 4 and shown in Fig. E. 1 at a common redshift of $z_{\mathrm{em}}=$ $0.28259 \pm 0.00005$, which are consistent with the absorption line redshift within errors. Their fluxes were measured through direct integration. From these measurements we derive key diagnostics of $\mathrm{H}$ II regions, such as extinction, SFR, and metallicity. Balmer lines are a good diagnostic for determining the level of extinction in $\mathrm{H}$ II regions. Their line ratio is purely determined by atomic constants. The observed $3 \sigma$ limit on the $\mathrm{H} \alpha / \mathrm{H} \beta$ flux ratio of $>1.9 \pm 0.11$ is consistent with the expected value of 2.76 for negligible extinction, when case B recombination is assumed. Since we have no indication otherwise, we use $A_{V \text {, host }}=0$ as a working hypothesis. Knowing that, the SFR at the explosion site is $0.037 \pm 0.002 M_{\odot} \mathrm{yr}^{-1}$, as measured from $\mathrm{H} \alpha$ using the relation in Kennicutt (1998) and correcting for a Chabrier initial mass function (IMF; Chabrier et al. 2000; Förster Schreiber et al. 2009). Since $H \beta$ is only marginally detected, we use the N2 diagnostic by Pettini \& Pagel (2004) to measure the metallicity. This oxygen abundance of $12+\log \mathrm{O} / \mathrm{H} \geq 8.57 \pm 0.05$ corresponds to a very high metallicity of $Z=(0.8 \pm 0.1) Z_{\odot}$. The systematic error of this indicator is 0.18 dex (Pettini \& Pagel 2004).

\subsection{Host galaxy}

\subsubsection{Emission line diagnostics}

The X-shooter spectrum of the host galaxy's nucleus (obtained 0.7 days after the GRB; see Fig. E.2) shows no absorption but a large number of emission lines at a common redshift of $z_{\mathrm{em}}=0.28256 \pm 0.00002$, as listed in Table 4 . Their fluxes were measured in the same fashion as at the explosion site.

Interestingly, the $\mathrm{H} \alpha$ emission line is significantly broader than any other emission line in the spectrum, where $F W H M(\mathrm{H} \alpha)=1.83 \pm 0.01 \AA$ but $F W H M(\mathrm{H} \beta)=1.22 \pm$ $0.05 \AA$. To elucidate the origin of the broadening, we followed Chatzopoulos et al. (2011, and references therein) and assumed three distinct models: i) thermal broadening, ii) single Thompson scattering of free electrons, and iii) multiple scattering of hot free electrons. In the first scenario, the proper motion of atoms leads to broadening that results in a Gaussian-shaped line profile. Since the flux of an emission line stems from the total flux of all star-forming regions, we additionally assume that there are two velocity components. The second is typical for a broad-lined region in an AGN, which produces exponential line profiles $(\propto \exp (-\Delta v / \sigma))$, where $\Delta v$ is the Doppler shift from the line centre and $\sigma$ is the velocity dispersion). The third describes dense media and produces Lorentzian line profiles.

The top right panel in Fig. 7 shows the $\mathrm{H} \alpha$ emission line with the best-fit model. The best-fit model consists of two
Gaussians centred at wavelengths $\lambda_{1}=8419.79 \pm 0.01 \AA$ and $\lambda_{2}=8419.73 \pm 0.03 \AA$ with FWHMs of $3.17 \pm 0.17 \AA$ $\left(\Delta v=112.9 \mathrm{~km} \mathrm{~s}^{-1}\right)$ and $1.38 \pm 0.04 \AA\left(\Delta v=49.1 \mathrm{~km} \mathrm{~s}^{-1}\right)$ and amplitudes of $(2.27 \pm 0.16) \times 10^{-16}$ and $(3.04 \pm 0.18) \times$ $10^{-16} \mathrm{erg} \mathrm{cm}^{-2} \mathrm{~s}^{-1}$, respectively. The width of the narrow component is consistent with the width of the other lines. The $\mathrm{S} / \mathrm{N}$ of $\mathrm{H} \beta$ is not sufficient to resolve both velocity components. We therefore simultaneously re-fit the $\mathrm{H} \alpha$ and $\mathrm{H} \beta$ lines assuming no offset between the two velocity components and using the width of each velocity component as a free but shared parameter. The best-fit values of the $\mathrm{H} \alpha$ line are identical to the aforementioned measurements within errors. For $\mathrm{H} \beta$, we measure a line flux of $(-0.13 \pm 0.27) \times 10^{-16} \mathrm{erg} \mathrm{cm}^{-2} \mathrm{~s}^{-1}$ and $(1.41 \pm 0.27) \times 10^{-16} \mathrm{erg} \mathrm{cm}^{-2} \mathrm{~s}^{-1}$ for the broad and narrow component, respectively.

To check for the possible contribution of an AGN to the emission lines, we put the integrated line measurements of $\mathrm{H} \alpha \mathrm{H} \beta,[\mathrm{N} \mathrm{II}] \lambda 6584$, and [O III] $\lambda 5007$ in the BPT diagnostic plot (Fig. 14; Baldwin et al. 1981). The emission-line ratios are fully consistent with those expected from star-formation. Knowing that, we use the Balmer decrements to measure the extinction. The Balmer decrements of the broad and the narrow component are consistent with negligible dust extinction. The inferred SFRs (computed in the same way as for the explosion site) are $0.33 \pm 0.03 M_{\odot} \mathrm{yr}^{-1}$ and $0.48 \pm 0.03 M_{\odot} \mathrm{yr}^{-1}$ for the broad and narrow component, respectively. We note that our galaxy spectrum covers only the inner $1.34 \operatorname{arcsec}^{2}$ of the host galaxy, while the galaxy has an area of $4.71 \mathrm{arcsec}^{2}$, as measured from the FWHMs of the semi-major and semi-minor axes. To measure the total SFR of the host galaxy, as traced by $\mathrm{H} \alpha$, and hence corrected for slit losses, we scaled the spectrum with photometry. This commonly used approach assumes the spectrum to be self-similar. The N2 metallicity indicator is calibrated on integrated measurements. Therefore, we measure an integrated oxygen abundance of $12+\log \mathrm{O} / \mathrm{H}=8.43 \pm 0.01$. The metallicity of $Z=0.55 Z_{\odot}$ is a bit lower than that of the explosion site, though the two are consistent with each other within $2.5 \sigma$.

Levesque et al. (2012) carried out an independent study of the emission-line properties of the explosion site, the curved bridge connecting the galaxy's nucleus with the explosion site (Fig. 1), and the host's nucleus using Magellan's low-resolution LDSS3 spectrograph with two different position angles (PA = $50^{\circ}$ and $\left.141^{\circ}\right)$. They inferred a significant reddening between $E(B-V)=0.24-0.31$ mag towards the nucleus and explosion site. Our higher $\mathrm{S} / \mathrm{N}$ and higher spectral-resolution data reveal that there are two dominant populations of star-forming regions at the nucleus but no evidence for reddening. The line measurements in Levesque et al. (2012) were corrected for this apparent reddening. If the extinction correction is undone, their values agree with ours within $\sim 3 \sigma$ for most lines. Some differences do exist though. They used a fixed aperture of 1 '. 14 , while we based the aperture size on the FWHM of the spectral PSF of the galaxy nucleus and the explosion site, where $F W H M$ (nucleus) $=11^{\prime \prime} 34$ and $F W H M(\mathrm{GRB})=0 .{ }^{\prime} 86$. As described in Sect. 2.1, we also ensured absolute flux-calibration and checked for differential flux losses by scaling the spectra to photometry.

The correction for the apparent extinction significantly overestimates the SFR measurements: Levesque et al. (2012) derived a value of $S F R \gtrsim 2.7 M_{\odot} \mathrm{yr}^{-1}$, while our total measurement is $0.81 M_{\odot} \mathrm{yr}^{-1}$. As we show in the following section, the SED fit of the host galaxy agrees with our spectroscopy results but is in conflict with theirs. Furthermore, our data show no evidence for the claimed asymmetries in the emission-line profiles (see Figs. 7, E.1 and E.2), in contrast to Levesque et al. (2012). The 

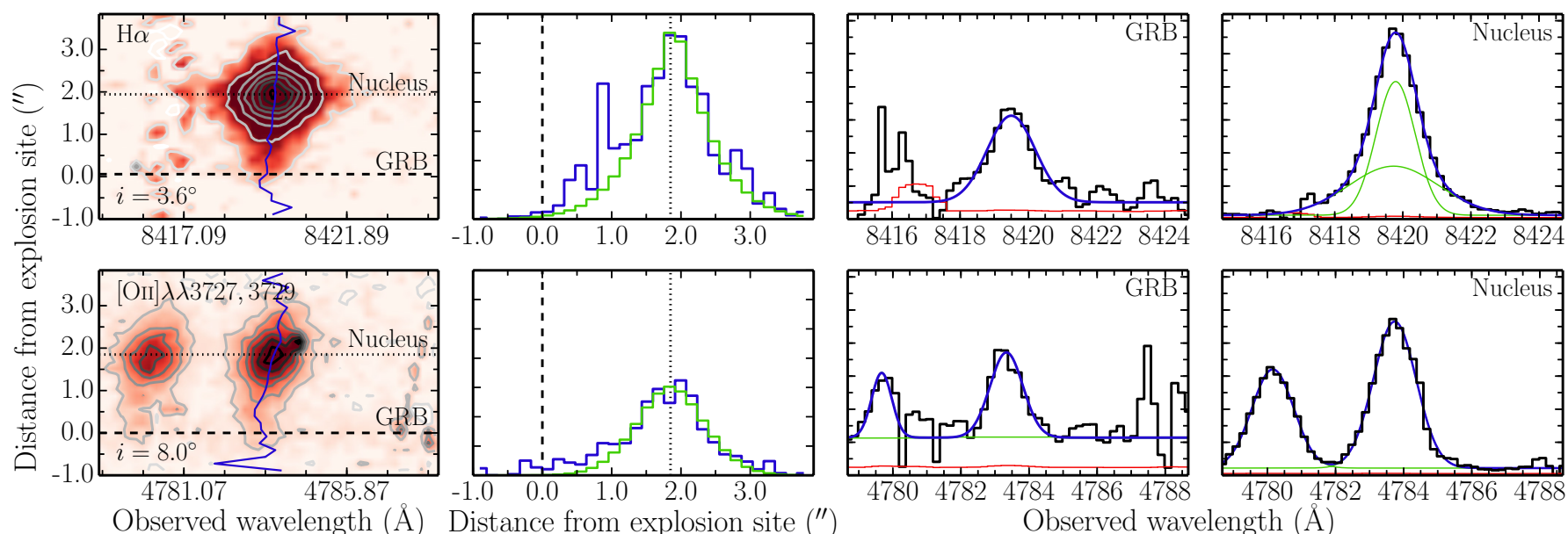

Fig. 7. Parts of the rectified and wavelength-calibrated 2D X-shooter spectrum $\left(\mathrm{PA}=41^{\circ}\right)$ obtained 0.72 days after the explosion $($ Table 1$)$. The first column shows the 2D profile of $\mathrm{H} \alpha$ and the [O II] doublet. The blue lines trace the position of maximum flux. The inclination angles $i$ (defined as the angle between the major axis and a vertical line) are displayed in the lower left corner. Contour lines are overlaid to guide the eye. The crossdispersion profiles (shown in blue) are displayed in the second column. The coding of the vertical lines is identical to that in the first column. For illustration purposes, we fitted the profiles with a Sérsic function (displayed in green), where the wings left of the centres of lines were excluded from the fit. The line profiles in dispersion direction are shown in the last two columns. The green line is the fit of individual components and the blue line of the compound. At $z=0.283$, an angular distance of $1^{\prime \prime}$ translates into a projected distance of $4.3 \mathrm{kpc}$. The error spectra in the third and fourth columns are overlaid in red.

nominal values for the skewness parameter are $0.06-0.21$ with a significance of $<2.1 \sigma$ at the explosion site and $-0.03-0.1$ with a significance of $<1.7 \sigma$ at the galaxy's nucleus.

\subsubsection{Morphology and SED}

The X-shooter spectrum from 2012 April 23 (PA = 41 ${ }^{\circ}$; Fig. 1) reveals that the most prominent nebular lines, which are the [O II] doublet and $\mathrm{H} \alpha$, extend from the galaxy's nucleus to the explosion site and slightly beyond (see Fig. 7). To obtain a better understanding of the peculiarity of the explosion site and the host morphology, we extracted their cross-dispersion profiles by fitting each row with a Gaussian (i.e. slicing the galaxy in chunks of 0 ! $15 \times 0 . ' 9$, which is equivalent to an area of $0.64 \times 3.9 \mathrm{kpc}^{2}$ at $z=0.283)$. The largest fluxes are recorded at the galaxy's nucleus (second column in Fig. 7), while the flux at the GRB site is very low. Since neither line of sight is affected by reddening in the host galaxy, the difference in $\mathrm{H} \alpha$ flux directly translates into a SFR difference. Hence, the explosion site does not show an enhanced SFR with respect to its surroundings and the nucleus. A fit of the cross-dispersion profiles with a Sérsic function (Col. 2 in Fig. 7) reveals an excess from the nucleus towards the GRB site. The excess in [O II] is more diffuse and extends to larger galactocentric radii. A possible explanation could be that this nebular line is in general less tightly correlated with starformation and affected by differences in ionisation, metallicity, and dust content (for a detailed discussion, see e.g. Kewley et al. 2004). We also note that the $2 \mathrm{D}$ profiles are slightly slanted. We measure a velocity difference between the galaxy's nucleus and the explosion site of 7 and $22.6 \mathrm{~km} \mathrm{~s}^{-1}$ at $\mathrm{H} \alpha$ and [O II] $\lambda 3729$, respectively. Strictly speaking, these are lower limits because this $\mathrm{X}$-shooter spectrum does not fully cover the nucleus.

An image of the host galaxy obtained 3.6 days after the explosion, shown in Fig. 1, reveals a curved bridge of emission connecting the transient with the host. The curved bridge was also covered by the slit of the $\mathrm{X}$-shooter afterglow spectrum from 2013 April 23 (Fig. 7). Even there, stars are formed at a rate that is in between that of the galaxy nucleus and the explosion site.
The GRB could therefore have occurred in either a morphologically disturbed/irregular galaxy, within an interacting companion, or in a spiral arm (however, no counter arm is visible on the opposite side of the galaxy).

Table 5 lists the brightness of the GRB host galaxy from 360 to $2140 \mathrm{~nm}$. We modelled the SED with Le Phare (Arnouts et al. 1999; Ilbert et al. 2006) ${ }^{17}$, using a grid of galaxy templates based on Bruzual \& Charlot (2003) stellar population-synthesis models with the Chabrier IMF and a Calzetti dust attenuation curve (Calzetti et al. 2000). For a description of the galaxy templates, physical parameters of the galaxy fitting and their error estimation, we refer to Krühler et al. (2011a). To account for zeropoint offsets in the cross calibration and absolute flux scale, a systematic error contribution of 0.05 mag was added in quadrature to the uncertainty introduced by photon noise. Figure 8 displays the observed host SED and its best fit. The observed SED is best described by a low-mass, barely-extinguished star-forming galaxy with a very young starburst (see Table 7). The extracted attenuation and SFR are consistent with results from emissionline diagnostics.

\subsection{GRB host galaxy environment}

In the previous section, we briefly mentioned the possibility that the true host could be a smaller galaxy interacting/merging with the $r^{\prime}=21 \mathrm{mag}$ galaxy. To explore this scenario further, we studied the nature of other objects in the vicinity of the GRB to find evidence for a galaxy over-density or galaxy interaction. Our GTC spectrum from 2012 April 25 showed that object G1 is at the same redshift as the GRB $(z=0.2828$; Figs. 1, D.1; Table 6). The angular distance of 7". 1 corresponds to a projected distance of $28.7 \mathrm{kpc}$ at $z=0.283$ from the host galaxy's nucleus. Intriguingly, we detect a curved arm of emission, though not fully recovered, that connects G1 with the GRB host in our deep Gemini and Liverpool Telescope images (Fig. 1). The blue colour of the tidal arm points to recent star formation. With G1's

${ }^{17}$ http://www.cfht .hawaii.edu/ arnouts/LEPHARE 


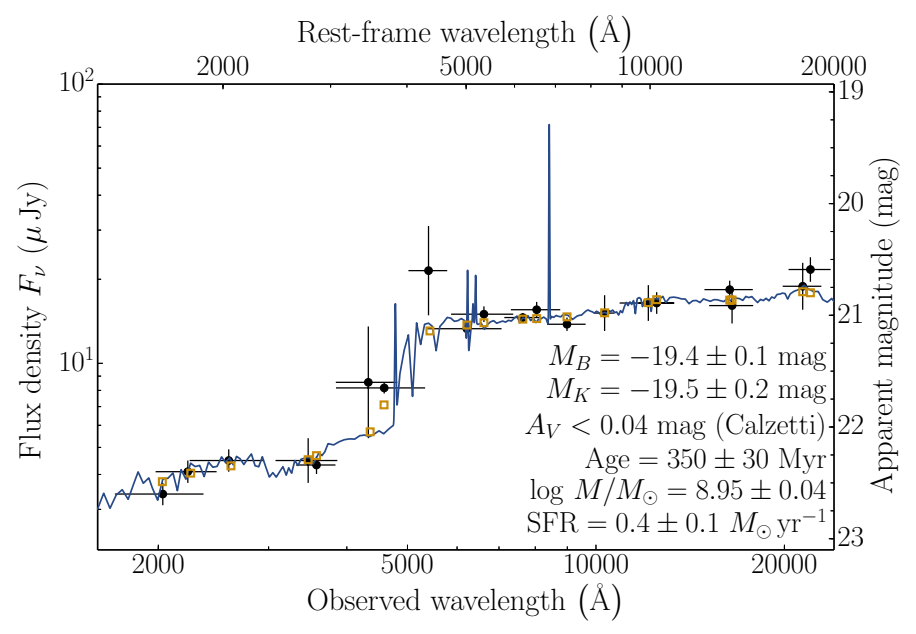

Fig. 8. Spectral energy distribution of the GRB host galaxy from 1600 to $24000 \AA$. The solid line displays the best-fit model of the SED with Le Phare $\left(\chi^{2}=24.7\right.$, number of filters $\left.=20\right)$. The beige open squares are the model predicted magnitudes.

blue colour, we have compelling evidence that both galaxies are interacting. This could be an indication that the arm connecting the host's nucleus to the GRB site is not a spiral arm but another tidal arm due to interaction of the $r^{\prime}=21$ mag galaxy with another galaxy. Deep HST observations are needed to investigate this issue further.

To map the star-formation activity inside the host galaxy and identify more galaxies at the GRB redshift up to distances of hundreds of $\mathrm{kpc}$, we acquired a deep image with a tuneable filter $(F W H M=15 \AA)$ that is centred at $\mathrm{H} \alpha$ at $z=0.283$ with the GTC 25.5 days after the GRB. Figure 9 shows a $33^{\prime \prime} \times 33^{\prime \prime}$-wide post stamp. The left panel was obtained with the $15 \AA$ wide tuneable filter; that is, it contains the emission from the $\mathrm{H} \alpha$ line and the continuum emission. The continuum, displayed in the middle panel, was imaged with a broadband filter centred at $8020 \AA$ (width $513 \AA$ ) that does not cover $\mathrm{H} \alpha$. The SN continuum is not highly variable, in neither the spectral range of the broadband filter nor at $\mathrm{H} \alpha$ (Fig. 4); the same is true for the host galaxy (Fig. 8). Hence, the difference image of both observations shows the pure $\mathrm{H} \alpha$ emission (right panel).

We detect four galaxy candidates that show excess emission at $8422 \AA$ (i.e. $\mathrm{H} \alpha$ redshifted to $z=0.283$; Fig. 9, Table 6). We identify the closest one, which is located at $7.8 \mathrm{kpc}$ of the GRB, as the host. The galaxy G1 (23 kpc from the centre of the host galaxy), already identified with the GTC spectrum from 25 April, is a satellite galaxy. Fitting its SED reveals G1 to be a smaller but more dusty version of the host galaxy (Fig. D.1, Table 6). The UV continuum of galaxy G2, shown in Fig. D.2, is poorly sampled. This results in a not well-constrained photo- $z$ of $z=0.23_{-0.10}^{+0.27}$ (99\% c.l.). The only strong emission line falling in that redshift interval is $\mathrm{H} \alpha$ at $z=0.283$. We therefore conclude that $\mathrm{G} 2$ is at the same redshift as the GRB. In contrast, galaxy G3 is a moderately high star-forming galaxy at $z \sim 0.66$ (Fig. D.3, Table 6). Most likely, either $\mathrm{H} \beta$, [O III] $\lambda 4960$ or [O III] $\lambda 5007$ fell in the passband of the tuneable filter.

\section{Discussion}

\section{1. $S N 2012 b z$}

In Sects. 3.1 and 3.3, we presented the properties of the GRB$\mathrm{SN}$. The initial UV/optical emission until $10 \mathrm{ks}$ is dominated
Table 5. Brightness of the GRB host galaxy in the optical/NIR.

\begin{tabular}{cccccc}
\hline \hline Filter & $\begin{array}{c}\lambda_{\text {center }} \\
(\AA)\end{array}$ & $\begin{array}{c}\text { Brightness } \\
(\mathrm{mag})\end{array}$ & Filter & $\begin{array}{c}\lambda_{\text {center }} \\
(\mathrm{nm})\end{array}$ & $\begin{array}{c}\text { Brightness } \\
(\mathrm{mag})\end{array}$ \\
\hline$w 2$ & 2033 & $22.60 \pm 0.10$ & $i^{\prime}$ & 7640.1 & $21.02 \pm 0.05$ \\
$m 2$ & 2229 & $22.40 \pm 0.10$ & $I$ & 8040.8 & $20.95 \pm 0.07$ \\
$w 1$ & 2591 & $22.30 \pm 0.10$ & $z^{\prime}$ & 8989.3 & $21.08 \pm 0.06$ \\
$u$ & 3501 & $22.30 \pm 0.20$ & $Y_{\mathrm{CAHA}}$ & 10322.8 & $20.98 \pm 0.16$ \\
$u^{\prime}$ & 3578.8 & $22.34 \pm 0.08$ & $J_{\text {CAHA }}$ & 12124.1 & $20.89 \pm 0.16$ \\
$b$ & 4329 & $21.60 \pm 0.50$ & $J_{\mathrm{UKIRT}}$ & 12502.4 & $20.89 \pm 0.10$ \\
$g^{\prime}$ & 4589.8 & $21.65 \pm 0.05$ & $H_{\mathrm{UKIRT}}$ & 16353.5 & $20.77 \pm 0.08$ \\
$v$ & 5402 & $20.60 \pm 0.40$ & $H_{\mathrm{CAHA}}$ & 16495.9 & $20.91 \pm 0.16$ \\
$r^{\prime}$ & 6219.6 & $21.12 \pm 0.04$ & $K_{\mathrm{CAHA}}$ & 21389.7 & $20.74 \pm 0.21$ \\
$R$ & 6623.0 & $20.99 \pm 0.07$ & $K_{\mathrm{UKIRT}}$ & 22004.5 & $20.59 \pm 0.11$ \\
\hline
\end{tabular}

Notes. The brightness was measured within a circular aperture (diameter 2'.5). The brightness was measured in $w 2 m 2 w 1 u b v$ with Swift/UVOT, in $u^{\prime} R I$ with NOT, in $g^{\prime} r^{\prime} i^{\prime} z^{\prime}$ with GROND, in $Y J H K$ with CAHA, and in $J H K$ with UKIRT.

by the thermal emission of the cooling stellar envelope after the shock break-out. About $1.4 \mathrm{hr}$ after the GRB, the stellar envelope had a temperature of $16 \mathrm{eV}$ and a radius of $7 \times 10^{13} \mathrm{~cm}$ (both in the observer frame). By modelling the radioactively powered light curve, we obtained $M_{\mathrm{Ni}}=0.40 M_{\odot}, M_{\mathrm{ej}}=4.72 M_{\odot}$, and $E_{\mathrm{k}}=3.29 \times 10^{52} \mathrm{erg}$. When the NIR emission is included, the nickel and ejecta masses would increase by 45 and $25 \%$, respectively, and the kinetic energy by $25 \%$. These values are among the highest recorded for GRB-SNe (Cano 2013). We computed the intrinsic $V$-band luminosity through direct integration over the SN spectrum. SN 2012bz has an absolute $V$-band peak magnitude of -19.7 mag, making it 0.3 mag more luminous than SN 1998bw. The phenomenological modelling of the SN light curve gave a similar value. In the $r^{\prime}$ band that overlaps with the rest-frame $V$ band, we inferred that the $\mathrm{SN}$ to be 0.3 mag brighter than SN 1998bw but evolved slightly faster. In the following, we discuss the SN properties in the context of other GRB-SNe.

\subsubsection{SN 2012bz in the context of other GRB-SNe}

Figure 10 shows the comparison of SN 2012bz at two different phases for which simultaneous spectra of SNe 1998bw, 2006aj, and 2010bh are available, all of which accompanied low- $L$ GRBs (Patat et al. 2001; Pian et al. 2006; Bufano et al. 2012). Overall, the spectra are very similar and show the same features, although line strengths and expansion velocities vary from object to object. We have illustrated this by annotating the main features as they have been identified in the past (e.g. Patat et al. 2001): Fe II, usually visible between 4500-5000 А; Si II, around 5600-6100 А; the Ca II IR triplet (that for SN 2012bz is in a noisy part of the spectrum between the VIS and the NIR arms; see lower panel); and possibly He I or Na I at around $5500 \AA$ (e.g. Bufano et al. 2012). We stress that these SNe have very large explosion velocities and that their broad lines are likely the result of blending that hampers the identification of the dominating line species producing the absorption feature, unless detailed modelling of the SN photosphere is carried out.

Nevertheless, the spectra of different GRB-SNe displayed in Fig. 10 are remarkably similar, reinforcing the idea that the nature of these blends, whatever it is, is the same for all GRB-SNe and pointing towards similar explosions. Differences do, however, exist in the expansion velocities (see Fig. 5). The spectra 


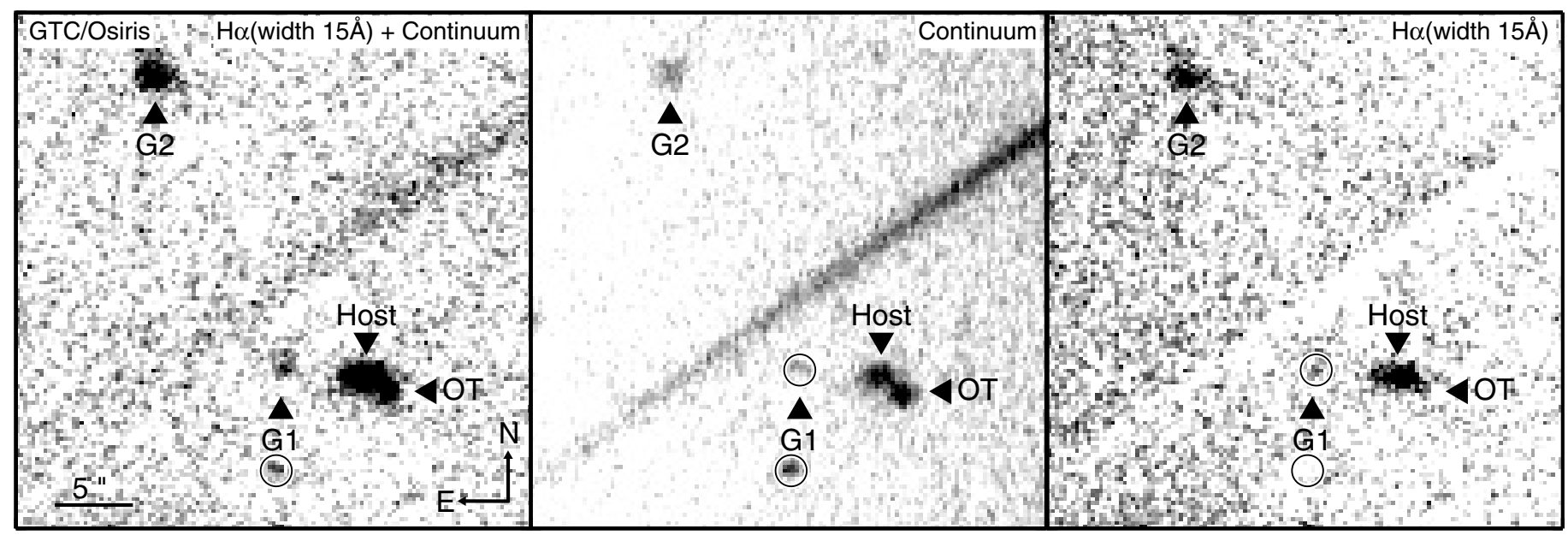

Fig. 9. Galaxy environment of GRB 120422A. The field size is $33^{\prime \prime} \times 33^{\prime \prime}$. The left panel shows the H $\alpha$ image (15 $\AA$ wide), which includes the emission line and the continuum. The middle panel shows the continuum centred at $8020 \AA$ ( $6250 \AA$ rest frame) to avoid the emission from H $\alpha$ (obtained with a 513- $\AA$ wide filter). The right panel is the subtraction of the left and middle panel, which is a pure H $\alpha$ image. The host, G1, and G2 are at the same redshift as the GRB (OT). Their projected distances are 7.3, 28.7, and $107.8 \mathrm{kpc}$ from the GRB site, respectively (see Table 6 for details). The diagonal stripes are produced by the $R=8.24$ mag foreground star that is $79^{\prime \prime} \mathrm{NW}$ of the explosion site. To illustrate the power of this observing method, we highlight an object south of G1 that does not have an emission line falling in the observed bandpass.

Table 6. Properties of emission-line galaxies detected with the tuneable filter.

\begin{tabular}{lllll}
\hline \hline & Host & G1 & G2 & G3 \\
\hline RA (J2000) & $09: 07: 38.5$ & $09: 07: 38.9$ & $09: 07: 39.4$ & $09: 07: 42.9$ \\
Dec (J2000) & $+14: 01: 08.46+14: 01: 09.12+14: 01: 27.83+14: 00: 15.40$ \\
Redshift & $0.28256^{a}$ & $0.2828^{a}$ & $0.283^{b}$ & $0.66_{-0.16}^{+0.02 c}$ \\
Projected & 7.3 & 28.7 & 107.8 & 584.5 \\
distance (kpc) & & & & \\
$M_{B}(\mathrm{mag})$ & $-19.4 \pm 0.1$ & $-17.1 \pm 0.1$ & $-18.7 \pm 0.1$ & $-21.5 \pm 0.1$ \\
$M_{K_{\mathrm{s}}}(\mathrm{mag})$ & $-19.5 \pm 0.2$ & $-17.9 \pm 0.2$ & $-19.1 \pm 0.2$ & $-22.4 \pm 0.2$ \\
$A_{V}(\mathrm{mag})$ & $<0.04$ & $\sim 0.6$ & $\sim 0$ & $\sim 0.6$ \\
$\log M_{\star}\left(M_{\odot}\right)$ & $8.95 \pm 0.04$ & $8.1_{-0.6}^{+0.3}$ & $8.8 \pm 0.1$ & $10.0_{-0.2}^{+0.1}$ \\
Age $(\mathrm{Myr})$ & $350 \pm 30$ & $460_{-380}^{+1520}$ & $1100_{-520}^{+750}$ & $730_{-250}^{+2470}$ \\
SFR $\left(M_{\odot} \mathrm{yr}^{-1}\right)$ & $0.4 \pm 0.1$ & $0.3_{-0.1}^{+0.2}$ & $0.7_{-0.2}^{+0.3}$ & $14.3_{-9.5}^{+10.1}$ \\
$\chi^{2} /$ n.o.f. & \\
\hline
\end{tabular}

Notes. The SEDs were fitted with Le Phare by assuming a Calzetti dust-attenuation law and a Chabrier IMF. For details, see Sect. 4.2.2. ${ }^{(a)}$ Spectroscopic redshift. ${ }^{(b)}$ The SED blueward of the Balmer break is poorly sampled, resulting in a not well-constrained photo- $z$ of $z=$ $0.23_{-0.10}^{+0.27}$ ( $99 \%$ c.l.). The only strong emission line falling in that redshift interval is $\mathrm{H} \alpha$ at $z=0.283$. The reported galaxy properties were obtained for that redshift. ${ }^{(c)}$ Photometric redshift. ${ }^{(d)}$ Projected distances are measured from the optical transient to the galaxies. The projected distance of G3 assumes $z=0.66$. ${ }^{(e)}$ "n.o.f." denotes the number of filters.

are displayed in an "expansion velocity sequence" going from the "fastest" (SN 2010bh; see also the discussion in Chornock et al. 2010; Bufano et al. 2012) to the "slowest" (SN 2006aj). This is at least true for the Fe II and Si II lines, and, in this respect, SN 2012bz seems intermediate and more similar to SN 1998bw. The Ca IR triplet shows a different velocity behaviour, which is not correlated with the one determined by the other elements, and SN 1998bw is clearly faster at all phases.

It is interesting to point out that the notch that has been possibly identified as He I, which could also be due to Na I (Bufano et al. 2012, see also Fig. 10 panel a), is also visible in SN $2012 \mathrm{bz}$ and in most optical GRB-SNe spectra with sufficient $\mathrm{S} / \mathrm{N}$ to the left of the main Si II trough. A powerful diagnostic to test the presence of He I is NIR spectroscopy (Patat et al. 2001; Bufano et al. 2012). Our X-shooter NIR spectra are unfortunately of low $\mathrm{S} / \mathrm{N}$, and for this reason, we focus our analysis only on the one obtained at maximum light (Fig. 10 lower panel). Still, however, this spectrum is dominated by a weak continuum, while most prominent features are located in unfavourable regions (the error spectrum is displayed). For comparison, we have also plotted an X-shooter spectrum of SN 2010bh obtained at a similar phase (Bufano et al. 2012). SN 1998bw does not have a contemporaneous spectrum but we show the one obtained at $T_{0}+33$ day, where the identified features are more clearly visible (Patat et al. 2001). Both the locations where one would expect to see He I $\lambda 10830$ or $\mathrm{C} I \lambda \lambda 10695,16890$ are located in very noisy atmospheric regions of our spectra at the redshift of SN $2012 \mathrm{bz}$, which prevents us from drawing any meaningful conclusion.

\subsubsection{A Phillips-type relation for GRB-SNe?}

Spectroscopy of GRB-SNe is in most cases limited to $z \lesssim 0.3$, which is the redshift domain that is observationally dominated by low- $L$ GRBs. At higher redshifts, the detection of a GRB-SN mostly depends on the detection of late red bumps that are modelled with a SN1998bw template (e.g. Zeh et al. 2004). In the past years, the sample of GRBs with detected late red bumps has been substantially increased (Zeh et al. 2004; Ferrero et al. 2006; Thöne et al. 2011; Cano 2013). We use these samples to compare the luminosity factor $k$ and the stretch factor $s$ of low- and high- $L$ GRBs. Among these, we only select those with meaningful values, which are either the GRB-SNe that have a clear bump in the light curve consistent with other GRB-SNe at the redshift of the GRB (rank "C" in Hjorth \& Bloom 2012) or spectroscopic evidence for the accompanying GRB-SNe (rank "B" and "A" in Hjorth \& Bloom 2012) ${ }^{18}$. Furthermore, most values were only obtained in the observed $R$ band. Since GRB-SNe have up to now been identified between $z=0.0085$ and $z \simeq 1$, the observed $R$ band probes different regions in the rest-frame. Supernovae

${ }_{18}$ For an updated list see:

http: //wwW. dark-cosmology.dk/GRBSN/GRB-SN_Table.html 

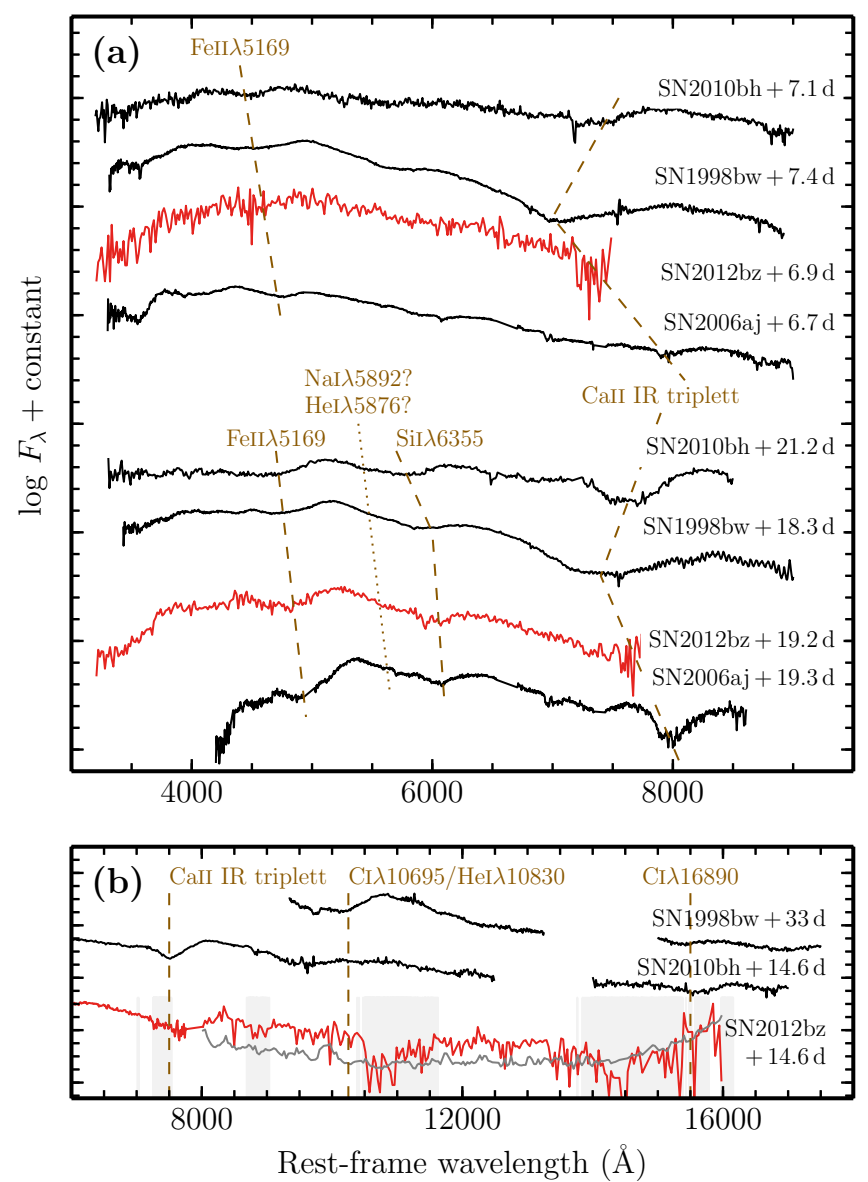

Fig. 10. a) Comparison of $\mathrm{SN} 2012 \mathrm{bz}$ (blue) to spectra of low- $L$ GRB SNe (black) at two different phases, $\sim 7$ and $\sim 20$ days past explosion, respectively. All comparisons are made in the rest frame. The dashed lines connect the approximate minima for the Fe II and Si II features, and the spectra are shown in an expansion velocity sequence from the fastest (SN 2010bh) to the slowest (SN 2006aj). A less significant (but real) feature that has been proposed to be He I or Na I is also identified. b) NIR arm of the X-shooter spectrum of SN 2012bz at maximum light (blue). The thin grey line is the error spectrum. The Ca II IR triplet at the redshift of SN 2012bz is located between the VIS and NIR arms. For comparison, NIR spectra of SN 1998bw and SN 2010bh are shown along with identification of the most prominent lines (Patat et al. 2001; Bufano et al. 2012). Unfortunately, these features fall in unfavourable noisy regions of our spectrum. Positions of atmospheric features (shifted to the redshift of GRB 120422A) are highlighted by the grey-shaded areas.

of GRBs emit most of their energy in the rest-frame $V$ band. Therefore, we only use those values where the observed bandpass partly overlaps with the rest-frame $V$ band (in the following called "quasi $V$ " band).

Figure 11 displays the parameter space of the nine GRB-SNe that fulfil both criteria. Supernovae of low- $L$ and high- $L$ GRBs occupy the same parameter space. Intriguingly, there is a trend between the luminosity and the stretch factor ${ }^{19}$. This is in line with recent findings by Hjorth (2013), who independently reported a correlation between the peak magnitude and the width of the peak. To estimate the correlation degree and significance,

\footnotetext{
${ }^{19}$ In the case of GRB-SNe, such a correlation was searched for by Ferrero et al. (2006) and Cano et al. (2011a) but not found. These studies disregarded the rest-frame waveband the data probed. In the case of Stanek et al. (2005), additionally the data set used was significantly smaller.
}

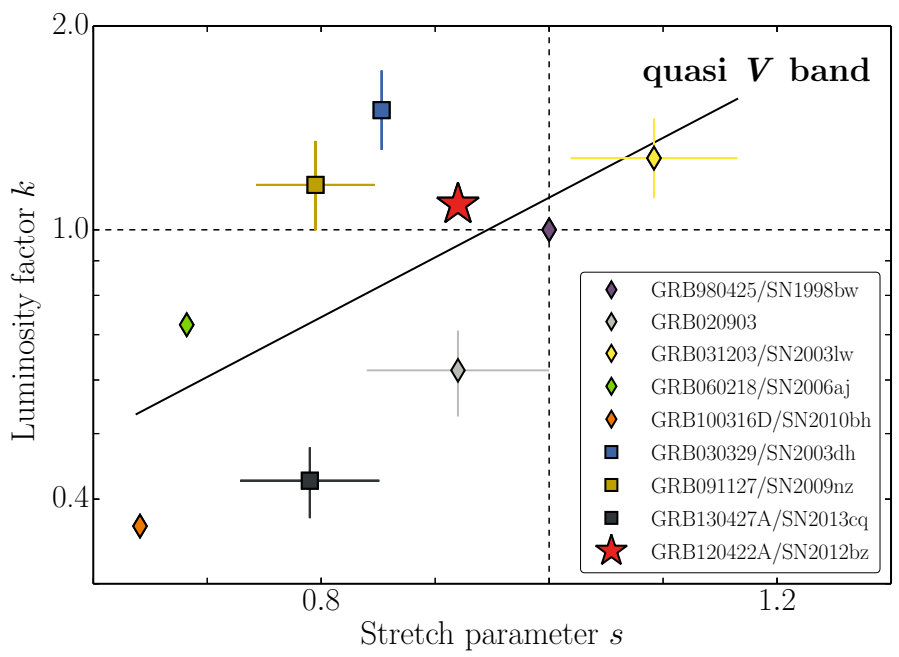

Fig. 11. SN luminosity factor $k$ (peak luminosity in units of SN 1998bw's peak luminosity) vs. stretch factor $s$ (time dilation vs. SN 1998bw's peak time) in the quasi $V$ band. Quasi rest-frame $V$ band means that the observed bandpass partly overlaps with the rest-frame $V$ band. Low- $L$ GRBs are highlighted by diamonds, high- $L$ GRBs by squares, and the intermediate- $L$ GRB $120422 \mathrm{~A}$ by a star. References: Ferrero et al. (2009): GRBs 020903, 030329, 031203, 060218, Kann (in priv. comm.): GRBs 091127 and 100316D, here: GRBs 120422A and 130427A.

we applied a Monte Carlo technique. In this method, every data point is represented by a 2D Gaussian, where the centre of peaks in each dimension is the parameter estimate, and the corresponding $1 \sigma$ errors are the width of the distributions. From these results, we construct 30000 resamples of the observed data sets, where each is obtained by a random sampling with replacement from the original data set. Note SN 1998bw was excluded since it is the reference value. For each of these data sets, we do a linear regression fit, using the model $\log _{10} k=A \times s+B$, and determine the correlation coefficients. The best fit values and their uncertainties are given by the centre and the width of the distribution functions. The best-fit values are $A=0.89 \pm 0.24$ and $B=-0.84 \pm 0.19$. The Pearson's correlation coefficient, Spearman's rank, and Kendal's $\tau$ give significances of $\sim 1.3 \sigma$. Despite the correlation being statistically not significant, the trend is similar to the Phillips relation (Phillips 1993), which builds the foundation for using Type Ia $\mathrm{SNe}$ as standard candles in cosmology.

The degree of correlation is affected by several systematics. First of all, none of the displayed $k, s$ values represent the true rest-frame $V$ band. To obtain the rest-frame $V$-band values, a more sophisticated approach is needed, which is beyond the scope of this paper. Systematic differences can arise because all GRB-SNe are broad-lined Type Ic SNe, but the evolution and the strength of absorption features depend on the specific GRB-SN (see Figs. 5, 10). Uncertainties in the line-of-sight extinction are the second largest source of error affecting $k$ but not $s$. For instance, the afterglow data of GRB 020903 are not good enough to build a SED for estimating the line-of-sight extinction. The extinction towards GRB 060218/SN 2006aj and GRB 100316D/SN 2010dh is very high and uncertain (Cano et al. 2011a; Bufano et al. 2012; Olivares et al. 2012). Furthermore, there are different approaches to how a 1998bw-template light curve for a specific band is constructed. Specifically, we measure a difference of 0.10 mag for GRB 120422A in the observed $r^{\prime}$-band peak magnitude between the methods by Zeh et al. (2004) and Cano (2013). The host 
contribution was considered either by image subtraction, subtraction of the nominal host flux, or addition of the host magnitude as a free parameter to the light curve fit for all GRB-SNe, except for GRB 130427A. Last but not least, the SN fit depends on how well the afterglow component is modelled. This affects $k$ as well as $s$.

\subsection{The afterglow of GRB $120422 A$}

Our analysis in Sect. 3.2 reveals: i) the optical (redward of $B$ band) and the NIR emission of the transient accompanying GRB 120422A is afterglow-dominated between $\sim 2$ and $60 \mathrm{ks}$; ii) the $\mathrm{X}$-ray emission is consistent with synchrotron radiation at all times (except for some small deviations during the first 200 s after the burst; Starling et al. 2012); iii) the initial Lorentz factor is $\Gamma_{0} \sim 50$; iv) the afterglow peak luminosity-density is $\lesssim 2 \times 10^{30} \mathrm{erg} \mathrm{s}^{-1} \mathrm{~Hz}^{-1}$; and v) the circumburst medium has constant density. Like the SN discussion, we put the afterglow in context of low- and high- $L$ GRBs.

As counterintuitive as it might be, a constant-density circumburst medium has been found around many GRBs (Schulze et al. 2011). van Marle et al. (2006) discussed several scenarios, such as a complex mass-loss history, a weak stellar wind, or a high pressure ISM, which can stall the undisturbed stellar wind close to a star. Employing the formalism in Schulze et al. (2011) and Eq. (4) in Chevalier \& Li (2000), we use the earliest $i^{\prime}$-band detections to infer a tentative limit of $<4 \times 10^{15} \mathrm{~cm}(0.001 \mathrm{pc})$ on the wind-termination shock radius, which is a very small value in the sample of Schulze et al. (2011).

Measurements of the initial Lorentz factor are limited to a small number of bursts with rapid follow-up. Typical values are about a few hundred for high- $L$ GRBs (Molinari et al. 2007; Ferrero et al. 2009; Greiner et al. 2009; Liang et al. 2010; Perley et al. 2011). For low- $L$ GRBs, measurements exist for 060218 (Soderberg et al. 2006a) and 100316D (Margutti et al. 2013). The inferred Lorentz factor of $\Gamma=1.5-2.3$ at 1 (GRB 100316D) and 5 days (GRB 060218) after the burst were obtained when the blast wave had already decelerated. According to Zhang \& Mészáros (2004, and references therein), a blast-wave's Lorentz factor evolves as $\Gamma \propto t^{-3 / 8}$ for a constant-density medium and and as $\Gamma \propto t^{-1 / 4}$ for a free-stellar-wind ambient density profile during the deceleration phase. Considering the time when the Lorentz factors were measured, the initial Lorentz factors were at most one order of magnitude larger, which is still smaller than that of GRB 120422A. This re-assures us in the identification of this phase transition and also illustrates the decrease in the blast-wave's velocity from high- to low- $L$ GRBs.

As mentioned in Sect. 3.2.3, the peak of an afterglow synchrotron spectrum crosses the sub-mm to $\mathrm{cm}$ range within the first week. During the first week, an afterglow can exhibit variability that can affect the peak flux and value of the injection frequency. Several sub-mm observations during the first week after the burst can be used as a proxy for the peak luminosity without the need for modelling the broadband afterglow (de Ugarte Postigo et al. 2012b). Figure 12 displays the inferred sub-mm peak fluxes as a function of redshift. The observed limit on GRB 120422A's peak flux-density from the sub$\mathrm{mm}$ observations and the limit from the SED modelling point to a faint afterglow. The limit of $\$ 2 \times 10^{30} \mathrm{erg} \mathrm{s}^{-1} \mathrm{~Hz}^{-1}$ is exceptionally deep for high- $L$ GRBs. For example, the afterglow of GRB 030329, a burst with spectroscopically confirmed SN and with $E_{\text {iso }}>10^{51} \mathrm{erg}$, had a peak luminosity-density that is $\sim 200$-times larger and the afterglow of GRB 080319B, a burst with photometric evidence for a SN (Tanvir et al. 2010), was

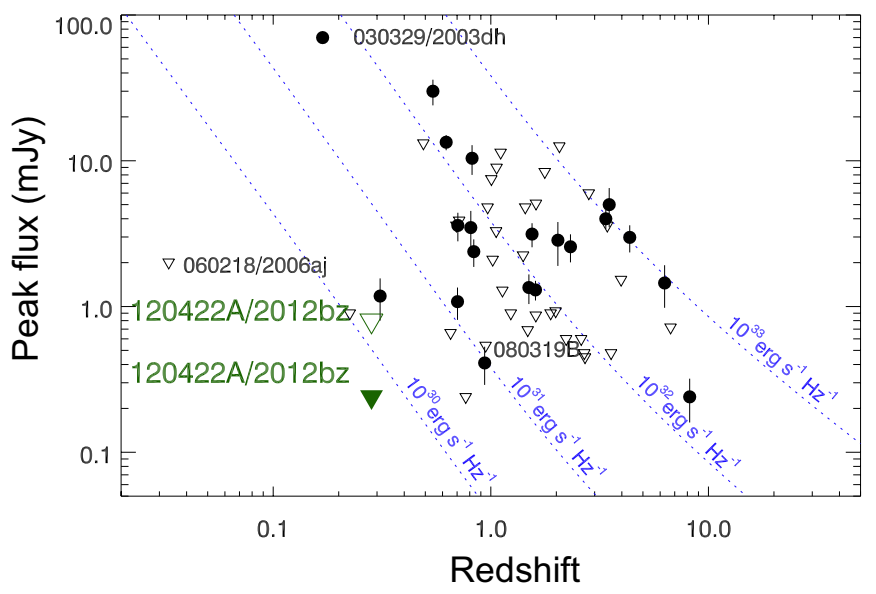

Fig. 12. Peak flux-density measured at sub- $\mathrm{mm} / \mathrm{mm}$ wavelengths vs. redshift. Triangles indicate $3 \sigma$ detection limits. The deepest observed limit on the peak flux-density of GRB 120422A is displayed by the filled green triangle, while the limit from the SED modelling is highlighted by the empty green triangle. Dotted lines display fluxdensity levels for equal luminosity at varying redshifts. Several interesting bursts are highlighted in the figure: the high- $L$ GRBs 030329 and $080319 \mathrm{~B}$ and the low- $L$ GRB 060218. Figure adapted from de Ugarte Postigo et al. (2012b).

about $~ 20$-times more luminous than 120422A. Intriguingly, the peak luminosity density is in the expected range of low- $L$ GRBs, such as GRB 060218.

Current sub-mm observations are limited to a small number of GRBs ( $\sim 5 \%$ of all GRBs) and have only been successful in detecting bright afterglows. The number of Swift GRBs with measured redshift is $\sim 27 \%$, which is $\sim 5$-times larger than the sub-mm/radio recovery rate and almost all them have a detected X-ray afterglow. de Ugarte Postigo et al. (2012b) reported on the existence of a correlation between the sub-mm flux density and the X-ray flux density at 0.5 days. Hence, we can re-address the question on the faintness of GRB 120422A's afterglow by exploring the X-ray luminosity distribution.

We downloaded the $0.3-10-\mathrm{keV}$ light curves of long Swift GRBs (i.e. $T_{90} \geq 2$ s) with detected X-ray afterglows (requiring at least two X-ray detections) and a known redshift from the Swift Burst Analyser (Evans et al. 2010) that were discovered between December 2004 and February 2014. Because of the small number of low- $L$ GRBs in the Swift sample, we include all pre-Swift-era GRBs with detected supernova (ranked better than " $C$ " in Hjorth \& Bloom 2012). We retrieve their light curves from the BeppoSAX GRB Afterglow Database (de Pasquale et al. 2006; Gendre et al. 2006 $)^{20}$. The differences in the observed bandpasses are considered when the X-ray luminosity in the $0.3-10-\mathrm{keV}$ rest-frame is computed. In total, 292 GRBs fulfil both criteria. Following Hogg et al. (2002) and assuming a tophat response function, the luminosity between 0.3 and $10 \mathrm{keV}$ is given by

$L_{(0.3-10) \mathrm{keV}}=4 \pi d_{\mathrm{L}}^{2}(z)(1+z)^{\beta-1} F_{(0.3-10) \mathrm{keV}}$,

where $d_{\mathrm{L}}$ is the luminosity distance and $\beta$ is the spectral slope. The Burst Analyser provides information on the spectral slope for each data point. Sometimes, the reported slope is highly variable or has unphysical values (for limitations of the Burst Analyser, see Evans et al. 2010), especially at late times ( $t>$ $1000 \mathrm{~s}$ ) when statistics are poor. To minimise the impact of such

${ }^{20}$ http://www.asdc.asi.it/GRBase/ 


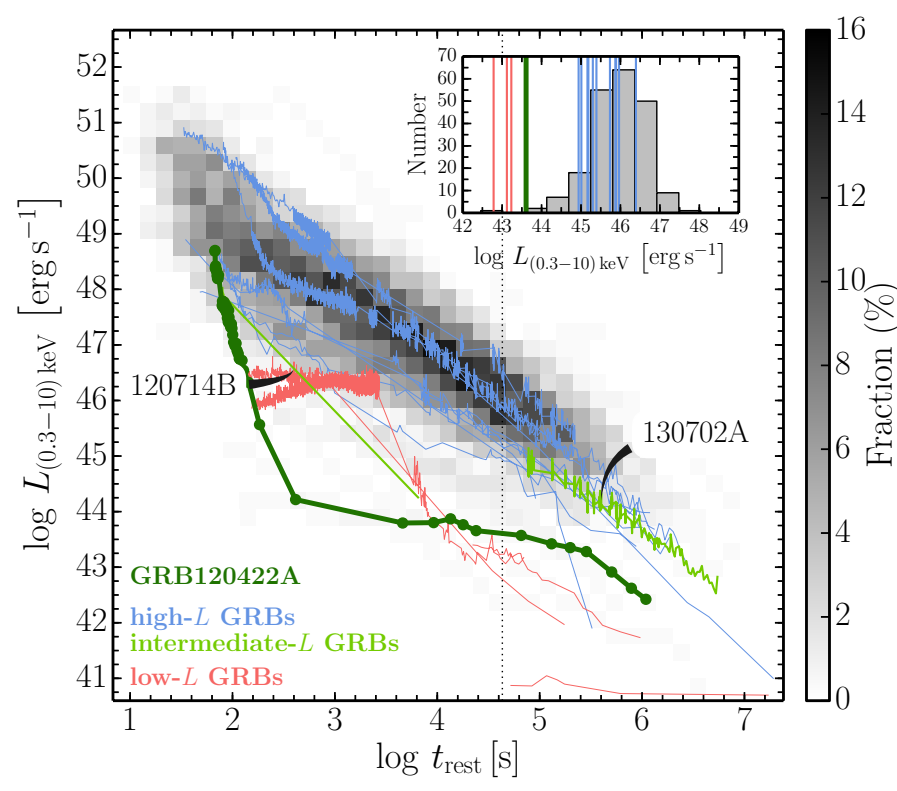

Fig. 13. X-ray light curves of low-, intermediate-, and high- $L \mathrm{SN}-$ GRBs. Overlaid is the evolution of the observed luminosity distribution of 273 long Swift GRBs for which a SN search was not feasible or unsuccessful (i.e. GRBs 060505 and 060614). These were discovered between December 2004 and February 2014. The colour table on the right side translates a grey shade at a given luminosity and time into a fraction of bursts. The inset displays the observed luminosity distribution at 0.5 days (dotted vertical line). The vertical lines in the inset show the luminosity of the intermediate- $L$ GRB $120422 \mathrm{~A}$ and the low- $L$ GRBs 031203, 060218, and 100316D.

artefacts, we set the spectral slope to the median late-time spectral slope (i.e. $t>1000 \mathrm{~s}$ ) if deviations are $<3 \sigma$ and if the slope is larger than 4. For pre-Swift GRBs, only time-average spectral slopes are available. From these data, we build a density plot by resampling the rest-frame X-ray light-curves to a grid that is defined by the observed luminosities of and the time interval spanned by all X-ray afterglows and interpolated between adjacent data points in case of data gaps.

The luminosity distribution as a function of rest-frame time is shown in Fig. 13 (the grey-shaded area). Highlighted in this plot are GRBs with detected SNe, colour-coded according to their time-averaged $\gamma$-ray luminosity. High- $L$ GRBs with detected $\mathrm{SNe}$ occupy the same parameter space as all high- $L$ for which no SN search was feasible (grey-shaded area). This supports the discovery made by $\mathrm{Xu}$ et al. (2013) that even bursts with the largest energy releases during the prompt $\gamma$-ray emission are accompanied by $\mathrm{SNe}$ (see also Tanvir et al. 2010), while intermediate- and low- $L$ GRBs are at the very faint end of the high- $L$ distribution. They even extend the observed high- $L$ distribution to much lower luminosities; for example, GRB 980425 was about 4.5 dex fainter than an average high- $L$ GRB at $100 \mathrm{ks}$. To quantify how faint intermediate- and low- $L$ GRBs are and to compare them to the peak-luminosity distribution, we build a histogram of 210 GRBs with detected X-ray afterglows at 0.5 days, which is presented in the inset of Fig. 13. The high- $L$ GRB distribution has a mean luminosity of $\left\langle\log L /\left(\mathrm{erg} \mathrm{s}^{-1}\right)\right\rangle=$ 45.92 and a dispersion of 0.69 dex. Among the intermediate$L$ GRBs, only GRB 120422 A was detected in the X-rays at 0.5 days. Compared to high- $L$ GRBs, GRB $120422 \mathrm{~A}$ is 2.3 dex fainter than the mean value, whereas its luminosity is only a factor of a few greater than the brightest low- $L$ GRBs, which is in line with the results from the sub-mm.

\subsection{The host galaxy and galaxy environment}

Our analysis in Sect. 4 reveals the following i) negligible extinction at the explosion site and at the galaxy nucleus; ii) two populations of H II regions in the nucleus; iii) a very low SFR at the explosion site; iv) a value close to solar metallicity at the explosion site and at the nucleus; v) the interaction of the host galaxy with a galaxy at a projected distance of $23 \mathrm{kpc}$; and vi) evidence for a galaxy group environment. At first, we discuss the GRB environment in the context of all GRBs, then the host galaxy and, finally, the galaxy environment.

The environment of GRB 120422A appears to be rather average. The lower limit on the $\mathrm{Mg}$ II column density of $\log N>$ 13.8 is $\sim 1$ dex lower than that of an average GRB environment (Christensen et al. 2011). However, such a low column density was reported for other GRBs before: $050922 \mathrm{C}$ had a value of $\log N=14.6 \pm 0.3$ (Piranomonte et al. 2008), and 121019B had $\log N=13.43_{-0.10}^{+0.08}$ (Sparre et al. 2011). Even lower values were found, such as $\log N=12.96_{-0.18}^{+0.12}$ for GRB 070125 (De Cia et al. 2011) and $\log N>12.6$ for GRB 071003 (Perley et al. 2008). To quantify the integrated absorption-line strength of the interstellar medium in GRB host galaxies, de Ugarte Postigo et al. (2012a) introduced the line-strength parameter (LSP) that is derived from EW measurements of detected absorption lines. The observed LSP of $-0.15 \pm 0.40$ is small, but the value is consistent with the mean for GRB environments, considering the large error.

In contrast, the negligible reddening with the value close to solar metallicity is remarkable (Table 7). The high metallicity is challenging for the well-accepted collapsar model (Woosley 2012, and references therein) that requires metal-poor stars but is viable in binary scenarios (e.g. Fryer \& Woosley 1998; Detmers et al. 2008), which predict that even massive, metal-rich stars in tight binary systems can form a long GRB. However, we note that the X-shooter spectrum of the explosion site probes an area of $0.64 \times 3.9 \mathrm{kpc}^{2}$. Based on HST observations, Fynbo et al. (2000) showed that the stellar-cluster hosting the GRB 980425 progenitor was very compact (the radius being $2.25 \mathrm{pc}$ ) and faint, and at lower spatial resolution, it would merge with a much brighter Wolf-Rayet-star-hosting complex that is $800 \mathrm{pc}$ away (see also Le Floc'h et al. 2006; Michałowski et al. 2014). Therefore, the possibility that the GRB occurred in a metal-poor environment cannot be rejected.

To investigate this peculiarity further, we compare the $[\mathrm{O}$ III $] / \mathrm{H} \beta$ vs $[\mathrm{N} \mathrm{II}] / \mathrm{H} \alpha$ line ratios with those of other GRB hosts (see Fig. 14). We furthermore distinguish between spatiallyresolved and integrated line measurements. The emission-line ratio of the host's nucleus is not different from other GRB hosts, apart from its exceptionally high metallicity. All hosts are located in the region that is dominated by $\mathrm{H}$ II regions. Compared to models by Dopita et al. (2006), the observed line ratios always point to stellar populations with an age of a few million years and metallicities between 0.05 and $2 Z_{\odot}$, in contrast to the bulk of emission-line galaxies in the SDSS DR9 (Ahn et al. 2012). The host of GRB 120422A is among the most metal-rich GRB hosts. Taken at face value, the line ratios place the explosion site at the high-metallicity end of the observed distribution in a region, which is equally populated by $\mathrm{H}$ II regions and AGN. This is odd because of two reasons: i) the line ratio of the host nucleus is dominated by the H II region and ii) the GRB explosion site is $7.3 \mathrm{kpc}$ off the galactic centre; that is, we would not expect a supermassive black hole so far off the galaxy centre. The large uncertainties in the line measurements do not allow drawing a firm conclusion on this peculiarity. 
Table 7. Properties of the host galaxy and GRB hosts at $z<1.5$.

\begin{tabular}{lccc}
\hline \hline Parameter & Host & GHostS & TOUGH \\
\hline Sample size & & 74 & 20 \\
Redshift & 0.28256 & $0.78_{-0.33}^{+0.23}$ & $0.83_{-0.44}^{+0.25}$ \\
$M_{\mathrm{UV}, \text { est }}(\mathrm{mag})$ & -18.2 & $<-18.6 \pm 1.2$ & $<-18.4 \pm 1.4$ \\
$M_{K_{\mathrm{s}} \text {,est }}(\mathrm{mag})$ & -19.8 & $<-20.6_{-0.9}^{+0.6}$ & $<-19.4_{-0.6}^{+0.7}$ \\
$M_{B, \mathrm{SED}}(\mathrm{mag})$ & $-19.4 \pm 0.1$ & $-20.5_{-1.0}^{+1.1}$ & $\ldots$ \\
$M_{K_{\mathrm{s}}, \mathrm{SED}}(\mathrm{mag})$ & $-19.5 \pm 0.2$ & $-20.2_{-0.9}^{+1.0}$ & $\ldots$ \\
$A_{V}(\mathrm{mag})$ & $<0.04$ & $0.6_{-0.2}^{+0.6}$ & $\ldots$ \\
$\log M_{\star}\left(M_{\odot}\right)$ & $8.95 \pm 0.04$ & $9.3 \pm 0.5$ & $\ldots$ \\
Age $(\mathrm{Myr})$ & $350 \pm 30$ & $1119_{-325}^{+836}$ & $\ldots$ \\
$S F R\left(M_{\odot} \mathrm{yr}^{-1}\right)$ & $0.4 \pm 0.1$ & $2.4_{-1.7}^{+4.0}$ & $\ldots$ \\
$Z\left(Z_{\odot}\right)$ & 0.6 & $0.5_{-0.1}^{+0.2}$ & $\ldots$ \\
Offset $(\mathrm{kpc})$ & 7.3 & $1.9_{-1.3}^{+1.2}$ & $\ldots$ \\
\hline
\end{tabular}

Notes. Host properties of $120422 \mathrm{~A}$ and the median values of GRB hosts, as compiled in the GHostS database (date: 2013 December 3) and of the homogeneous, optically unbiased TOUGH survey (Hjorth et al. 2012, incl. results from Schulze et al., in prep.). The errors of the comparison samples indicate the distance from the median values to the 25 and $75 \%$ percentiles. The age represents the age of the starburst. The stellar mass of GRB 120422A's host was calculated by assuming the Chabrier IMF. The UV and $K_{\mathrm{S}}$ luminosities, marked by "est" were computed using the method in Schulze et al. (in prep.) and Laskar et al. (2011), respectively. Measurements designated with "SED" were obtained from SED fitting. The GRB offsets of the sub-set of bursts in the GHostS sample are compiled in Bloom et al. (2002), and the age distribution and results from SED fitting for the GHost sample were taken from Savaglio et al. (2009).

Comparing the integrated host properties with GRB samples is not straightforward. Most samples are heterogeneous and biased towards a particular GRB population, such as GRBs with negligible reddening or bright afterglows. On the other hand, optically unbiased samples are limited to observations in a few bands, from which only a few host properties can be extracted. Keeping these limitations in mind, we compare GRB 120422A's host to the GHostS database, which was built by Savaglio et al. (2009). This contains well-sampled multi-band SEDs and host spectra for several hosts $(\sim 25)$, which have been selected in a heterogeneous way, and the optically-unbiased, homogeneous GRB host (TOUGH) sample by Hjorth et al. (2012), which is in most cases limited to observations in the $R$ and in the $K_{\mathrm{S}}$ bands. Based on recent findings by Perley et al. (2013) where GRB hosts at $z<1.5$ are bluer and significantly less massive than at higher redshifts, we limit the comparison to hosts at $z<1.5$.

Table 7 lists the host properties of 120422A and the median values for both comparison samples. In context of the GHostS sample, GRB 120422A's host is in the lower half of the luminosity, mass, and SFR distribution. Only its very low extinction (0.5 mag less than the GHostS median) in combination with high metallicity is peculiar. We caution that the completeness of both properties is very low. Another peculiarity is the exceptionally large distance between the explosion site and the galaxy's nucleus. The offset of $7.3 \mathrm{kpc}$ is among the largest values reported in Bloom et al. (2002). Most GRBs are found close to the centre of their host galaxies (Bloom et al. 2002) and in particular close to/in the UV-brightest regions (Fruchter et al. 2006). A similar picture can be drawn from the TOUGH survey. Since only observations in two filters are available for most hosts, we assume that the rest-frame NIR and UV at $1700 \AA$ can be approximated by power-laws, similar to the approaches in Laskar et al. (2011)

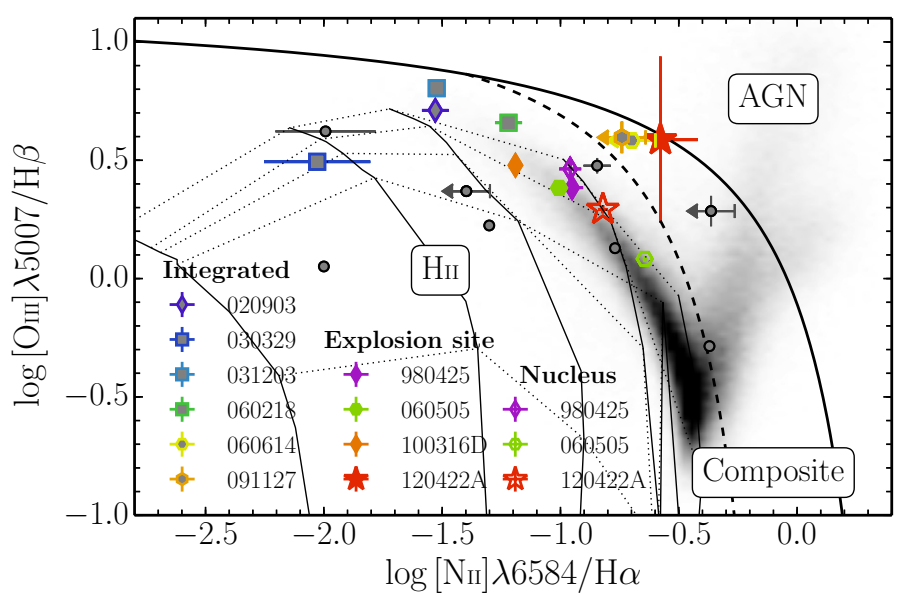

Fig. 14. Emission-line ratios for low- $L(\diamond)$ and high- $L(\square)$ GRB hosts and explosion sites. Long GRBs, for which a SN search was not feasible, are shown as circles. Limits are donated by arrows. For comparison, the emission-line ratios are overlaid for a wide population of field galaxies from the SDSS DR9 (Ahn et al. 2012) sample as a density plot. Among these data, we selected those whose emission lines were detected at $>5 \sigma$ c.l. The discerning line between star-formation and AGN-dominated galaxies is shown as a thick solid line and was taken from Kewley et al. (2001). The region of composite galaxies is encircled by the thick solid the thick dashed lines (Kauffmann et al. 2003). Evolutionary models, calculated by Dopita et al. (2006), that link emission-line regions at ages from 0.1 to $5 \mathrm{Myr}$ (shown as dotted lines; ages: $0.1,1,2,3$, and 4 Myr with the youngest stellar populations having the highest $[\mathrm{O} \mathrm{III}] / \mathrm{H} \beta$ line ratios) and different metallicities (shown as thin solid lines; $Z=0.05,0.2,0.5,1.0$, and $2.0 Z_{\odot}$; metallicity increases from left to right) are displayed. Errors are shown, if available. In some cases these are smaller than the size of the respective plot symbol. Figure adapted from Christensen et al. (2008). References: Della Valle et al. (2006a): the SN-less GRB 060614; Christensen et al. (2008): 980425, Han et al. (2010): 990712, 020903, and 030329; Levesque et al. (2010a): 991208, 010921, 050826, and 070612; Levesque et al. (2010b): 020819B; Thöne et al. (2008): the SN-less GRB 060505; Wiersema et al. (2007): 060218; Krühler et al. (2012): 080605A; Vergani et al. (2011): 091127; Levesque et al. (2011): $100316 \mathrm{D}$.

and in Schulze et al. (in prep.). We measure a UV luminosity at $1700 \AA$ of $-18.2 \mathrm{mag}$ and a $K_{\mathrm{s}}$-band luminosity of $-19.8 \mathrm{mag}$ (see Table 7). These values are consistent with the ensemble median values.

Observations with the tuneable filters revealed that the host is interacting with another galaxy that is at a projected distance of $23 \mathrm{kpc}$ from the host galaxy's nucleus and that there is a further star-forming galaxies at the same redshift within $\sim 110 \mathrm{kpc}$. In general, little is known about the galaxy environments of GRB host galaxies. Several GRB fields show an increased galaxy density, e.g. GRBs 000301C, 000926 (Fynbo et al. 2002), 011211 (Fynbo et al. 2003), 021004 (Jakobsson et al. 2005; Baryshev et al. 2010), 030226 (Jakobsson et al. 2005), 030115 (Levan et al. 2006), 050820A (Chen 2012), and 060505 (Thöne et al. 2008), but the nearest burst, GRB 980425, does not (Foley et al. 2006). The comparison is also limited due to the lack of information for SN fields.

\subsection{The missing link between low- and high-L GRBs}

The division between low- and high- $L$ GRBs is not entirely operational. Both populations have very distinct properties. Low- $L$ GRBs are thought to be driven by shock break-outs, 
producing (quasi-)spherical explosions whose $\gamma$-ray light curves are smooth and single-peaked (Campana et al. 2006; Starling et al. 2011; Nakar \& Sari 2012), spectra that can have peak energies of only a few keV (e.g. Campana et al. 2006; Kaneko et al. 2007; Starling et al. 2011), and mildly relativistic outflows ( $\Gamma<10$; e.g. Soderberg et al. 2006a Margutti et al. 2013). In contrast, high- $L$ GRBs are powered by ultra-relativistic collimated outflows with Lorentz factors of a few hundred (Molinari et al. 2007; Ferrero et al. 2009; Greiner et al. 2009; Perley et al. 2011) and $\gamma$-ray light curves that can exhibit variability in the milli-second domain (Bhat et al. 2012). The rate of low- $L$ GRBs in the nearby Universe exceeds that of high- $L$ GRBs by a factor of 10-1000 (Pian et al. 2006; Chapman et al. 2007; Guetta \& Della Valle 2007; Liang et al. 2007; Virgili et al. 2009; Wanderman \& Piran 2010). However, recent work by Lazzati et al. (2012) based on relativistic jet simulations propose a nonuniform distribution of the central engine's on-time to account for the differences.

GRB 120422A is one of the very few examples of intermediate-luminosity GRBs. Its $\gamma$-ray light curve exhibits an initial spike (starting at $T_{0}-3 \mathrm{~s}$ and ending at $\sim T_{0}+20 \mathrm{~s}$; Barthelmy et al. 2012) followed by a weaker and softer extended component (starting at $T_{0}+45 \mathrm{~s}$ and ending at to $T_{0}+65 \mathrm{~s}$; Barthelmy et al. 2012), as observed in other high- $L$ GRBs before (Bostanc1 et al. 2013). In addition, the X-ray emission is not dominated by thermal emission from the cooling photosphere after the shock break-out, like the low- $L$ GRBs 060218 and 100316D (Campana et al. 2006; Nakar \& Sari 2012). In contrast, the properties of the longer-lasting transient that accompanies the GRB point in a different direction. The blast wave had a very low initial Lorentz factor of $\Gamma_{0} \sim 50$, and the afterglow that was produced had a peak luminosity of $L_{v, \max } \lesssim 2 \times 10^{30} \mathrm{erg} \mathrm{s}^{-1} \mathrm{~Hz}^{-1}$, which is unprecedentedly low for a high- $L$ GRB. Thanks to the weak afterglow, the signature of the shock break-out was for the first time detected for a non-low- $L$ GRB.

The failed-jet model predicts high- $L$ GRBs to transition via low- $L$ bursts to engine-driven $\mathrm{SNe}$, as the jet produced by the central engine gets weaker, because of a lower kinetic energy in the outflow, a central engine that is active for a shorter period, or a less collimated outflow (Bromberg et al. 2011; Lazzati et al. 2012). According to this model, the weaker a jet, the more it gets decelerated in the stellar envelope until it is choked. Examples for choked jets are the Type Ib/c SNe 2002ap, 2009bb, 2012ap, and 2012au (Soderberg et al. 2006b, 2010; Milisavljevic et al. 2013; Margutti et al. 2013, 2014; Chakraborti et al. 2014) and the almost choked GRB 100316D, whose jet barely broke through the stellar cocoon (Margutti et al. 2013). Because we do detect the thermal emission from the cooling photosphere after the shock break-out, it raises the questions of how much energy GRB 120422A's jet has already transferred into the stellar envelope and how much more energy it could have lost before getting choked. As coined by Hjorth (2013), GRB 120422A is indeed a transition object between shock-break-out-driven low- $L$ and high- $L$ GRBs that are powered by ultra-relativistic jets.

To fully connect GRBs of low and high luminosities, it has to be shown that even the most luminous bursts $\left(L_{\text {iso }} \sim 10^{54} \mathrm{erg} \mathrm{s}^{-1}\right)$ are accompanied by SNe. These very energetic bursts have, however, been found at redshifts where SN searches are getting unfeasible; that is, $z \gtrsim 1$. Fortuitously, one of the most energetic bursts, GRB 130427A, occurred at $z=0.34$ (Perley et al. 2013; Xu et al. 2013). During its prompt $\gamma$-ray emission that had a duration of $T_{90}=276 \mathrm{~s}$, this burst released $8.1 \times 10^{53} \mathrm{erg}$ (Maselli et al. 2014). This translates into a time-averaged $\gamma$-ray luminosity of $L_{\text {iso }} \sim 10^{51.6} \mathrm{erg} \mathrm{s}^{-1}$. Thanks to its low redshift, an accompanying broad-lined Type Ic SN was spectroscopically detected with properties similar to those of low- $L$ GRBs (Xu et al. 2013, see also Tanvir et al. 2010, who reported on the photometric $\mathrm{SN}$ discovery for an almost 1 dex more luminous GRB). In addition, Maselli et al. (2014) showed that its afterglow properties are similar to those of very energetic, high-redshift GRBs, making it a genuine very high- $L$ GRB.

Combining the findings on GRBs 120422A and 130427A lets us conclude that low- and high- $L$ GRBs are not distinct populations of stellar explosions. They are due to the gravitational collapse of very massive stars and are accompanied by SNe. Their central engines are the same. Only the properties of their prompt emissions and of their afterglows (shock break-out vs. jet-dominated) differ, depending on whether the jet can successfully drill through the stellar cocoon. This does not make them disjunctive phenomena.

\section{Summary and conclusions}

We carried out extensive imaging and spectroscopy campaigns to study the intermediate-luminosity GRB 120422A that shares properties of low- $L$ and high- $L$ GRBs. Our detailed analysis focussed on the GRB-SN 2012bz, the GRB afterglow, the host galaxy, and its environment.

We showed that SN $2012 \mathrm{bz}$ is the most luminous spectroscopically-confirmed GRB-SN to date with a peak luminosity of $M_{V}=-19.7 \mathrm{mag}$. The explosion physics parameters of $M_{\mathrm{Ni}}=0.58 M_{\odot}, M_{\mathrm{ej}}=5.87 M_{\odot}$, and $E_{\mathrm{k}}=4.10 \times 10^{52} \mathrm{erg}$ are among the largest values known to date. However, the exact values highly depend on the NIR contribution. Cano et al. (2011a) showed that the nickel and the ejecta masses and the kinetic energy can be underestimated by $25-45 \%$ if the NIR is not included in the modelling of the bolometric light curve. For future GRBSN studies, it is imperative to secure NIR data to place more stringent constraints on GRB progenitor models. As an alternative to a campaign with long wavelength coverage, the method presented in Lyman et al. (2014) would allow us to construct the bolometric light curve from two optical light curves with welldetermined $k$-corrections.

The spectral sequence of the SN covers a time span of $\sim 40$ days. All spectra are similar to those of other GRB-SNe. Differences exist in the strength of the absorption features and expansion velocities. For the first time, Fe II $\lambda 5169$ was used to trace the evolution of the GRB-SN expansion velocity. The velocities and their evolution are not very different from Si II measurements. The advantages of Fe II $\lambda 5169$ are that it is easier to identify and it is detectable at earlier times. We find an intriguing trend between the peak luminosity $k$ and $\mathrm{SN}$ stretch factor $s$, which is similar to the Philips relation. Its significance is poor but several systematic errors affect the result.

GRB 120422A was accompanied by one of the least luminous afterglows detected to date. Its blast wave expanded with a low initial Lorentz factor of $\Gamma_{0} \sim 50$ into a constantdensity medium and produced a weak afterglow $L_{v \text {, max }} \lesssim 2 \times$ $10^{30} \mathrm{erg} \mathrm{s}^{-1} \mathrm{~Hz}^{-1}$. Thanks to the weak afterglow, we recovered emission from the cooling photosphere after the shock breakout, which was only observed for the low- $L$ GRBs 060218 and 100316D. The photosphere of GRB 120422A had a temperature of $T_{\mathrm{obs}} \sim 16 \mathrm{eV}$ and a radius of $R_{\mathrm{obs}} \sim 7 \times 10^{13} \mathrm{~cm}$ at $1.4 \mathrm{~h}$ after the GRB, which are typical values of SNe with detected shock break-out signatures. This fundamentally new quality for a non-low- $L$ GRB questions whether $120422 \mathrm{~A}$ is a genuine high- $L$ GRB. Considering the properties of the prompt emission and the afterglow makes us conclude that GRB 120422A is the missing 
link between shock-break-out-driven low- $L$ and high- $L$ GRBs that are powered by an ultra-relativistic jet, hence providing evidence for the failed-jet model for low- $L$ GRBs.

The GRB occurred in an almost typical host galaxy. Its closeto-solar metallicity, along with its negligible extinction, makes it peculiar. Thanks to the large offset of $7.3 \mathrm{kpc}$ from the nucleus, we perform spatially resolved spectroscopy. Surprisingly, even at the explosion site, the metallicity is close to solar, while the SFR is not enhanced with respect to its immediate environment and is only $1 / 10$ of that of the galaxy's nucleus. Based on the $\mathrm{N} 2$ indicator, we measure $Z=(0.8 \pm 0.1) Z_{\odot}$ at the explosion site. This does not necessarily mean that the GRB-hosting starforming region had these properties. The $\mathrm{X}$-shooter spectrum was only sensitive to a region of $4.0 \times 3.9 \mathrm{kpc}^{2}$. What needs to be stressed is that emission-line measurements from low-resolution spectra should be taken with caution. Our medium resolution data revealed that the $\mathrm{H} \alpha$ line is resolved into two components. This can lead to an overestimation of the extinction and SFR in low-resolution data.

Our narrow-band imaging (width $15 \AA$ ) showed that the host is possibly interacting with a galaxy that lies at a projected distance of $23 \mathrm{kpc}$ away. We identified an additional putative galaxy-group member within $110 \mathrm{kpc}$ from the GRB. In contrast to previous studies of GRB galaxy environments, tuneable filters allow us to more efficiently identify star-forming galaxies at a GRB redshift. In particular, this approach is complementary to SED fitting techniques, which are limited to bright galaxies but not necessarily highly star-forming galaxies. Both approaches are needed to address the long-standing questions on the peculiarity of GRB host galaxies and how galaxy interaction affects the production of GRB progenitors at low and high metallicities.

Acknowledgements. We thank Shri Kulkarni (Caltech) for obtaining the Keck spectrum. S.S. thanks Tsvi Piran (The Hebrew University, Israel), Nir Sapir, Eli Waxman (Weizmann Institute of Science, Israel), Milena Bufano (Universidad Andrés Bello, Chile), Maryam Modjaz (New York University, USA), and the anonymous referee for many productive and valuable discussions. S.S. acknowledges support by a Grant of Excellence from the Icelandic Research Fund, from the University of Iceland Research Fund, from the Dark Cosmology Centre, where part of this study was performed, and from CONICYT through FONDECYT grant 3140534. We acknowledge support from Basal-CATA PFB06/2007 (FEB, SS), Iniciativa Cientifica Milenio grant P10-064-F (Millennium Center for Supernova Science), by Project IC120009 "Millennium Institute of Astrophysics (MAS)" of Iniciativa Científica Milenio del Ministerio de Economía, Fomento y Turismo de Chile, with input from "Fondo de Innovación para la Competitividad, del Ministerio de Economía, Fomento y Turismo de Chile" (F.E.B., G.P., J.A.P., S.S.), CONICYT-Chile FONDECYT 1101024 (FEB). J.A.P. acknowledges support by CONICYT through FONDECYT grant 3110142. D.M. acknowledges the Instrument Center for Danish Astrophysics for support. T.K. and H.K. acknowledge support by the European Commission under the Marie Curie Intra-European Fellowship Programme in FP7. J.P.U.F., B.M.J., and D.X. acknowledge support from the ERC-StG grant EGGS-278202. K.L.P. acknowledges financial support by the UK Space Agency for the Swift project. G.L. is supported by the Swedish Research Council through grant No. 623-20117117. J.L. acknowledges the UK Science and Technology Facilities Council for research studentship support. The research activity of AdUP, C.T., and J.G. is supported by Spanish research project AYA2012-39362-C02-02. A.d.U.P. acknowledges support by the European Commission under the Marie Curie Career Integration Grant programme (FP7-PEOPLE-2012-CIG 322307). A.J.C.T. acknowledges support from the Spanish research project AYA2009-14000-C0301 and AYA2012-39727-C03-01 D.A.K. acknowledges support by the DFG cluster of excellence "Origin and Structure of the Universe" and funding by the Thüringer Landessternwarte Tautenburg. A.R. acknowledges support by the Thüringer Landessternwarte Tautenburg. P.S. acknowledges support through the Sofja Kovalevskaja Award from the Alexander von Humboldt Foundation of Germany. A.N.G. and S.K. acknowledge support by DFG KL 766/16-1. S. Schmidl acknowledges support by the Thüringer Ministerium für Bildung, Wissenschaft und Kultur under FKZ 12010-514. The Dark Cosmology Centre is funded by the Danish National Research Foundation. This work made use of data supplied by the UK Swift Science Data Centre at the University of Leicester.
This research has made use of the GHostS database (www.grbhosts.org), which is partly funded by Spitzer/NASA grant RSA Agreement No. 1287913. Based in part on observations collected at the European Organisation for Astronomical Research in the Southern Hemisphere, Chile, as part of the program 089.A-0067, the Gemini Observatory, which is operated by the Association of Universities for Research in Astronomy, Inc., under a co-operative agreement with the NSF on behalf of the Gemini partnership, as part of the programs GN-2012A-Q9, GN-2012A-Q-39, GN-2012B-Q-5, GS-2012A-Q-30, GS-2012A-Q-38, GS2012A-Q-30, and GN-2012B-Q-5, the Nordic Optical Telescope (NOT), operated by the Nordic Optical Telescope Scientific Association at the Observatorio del Roque de los Muchachos, La Palma, Spain, of the Instituto de Astrofisica de Canarias, as part of the program P45-002 (PI: Jakobsson) and ITP10-04 (PI: Kotak, QUB), the Gran Telescopio Canarias (GTC), installed in the Spanish Observatorio del Roque de los Muchachos of the Instituto de Astrofísica de Canarias, in the island of La Palma, with Magellan as part of CN2012A059, with the IRAM Plateau de Bure Interferometer, the James Clerk Maxwell Telescope, as part of the program M12AI12, with XMM-Newton, an ESA science mission with instruments and contributions directly funded by ESA Member States and NASA. Some of the data presented herein were obtained at the W.M. Keck Observatory, which is operated as a scientific partnership among the California Institute of Technology, the University of California and the National Aeronautics and Space Administration. The observatory was made possible by the generous financial support of the W.M. Keck Foundation. The United Kingdom Infrared Telescope is operated by the Joint Astronomy Centre on behalf of the Science and Technology Facilities Council of the UK The James Clerk Maxwell Telescope is operated by the Joint Astronomy Centre on behalf of the Science and Technology Facilities Council of the United Kingdom, the National Research Council of Canada, and the Netherlands Organisation for Scientific Research. Additional funds for the construction of SCUBA-2 were provided by the Canada Foundation for Innovation. The Submillimeter Array is a joint project between the Smithsonian Astrophysical Observatory and the Academia Sinica Institute of Astronomy and Astrophysics and is funded by the Smithsonian Institution and the Academia Sinica. IRAM is supported by INSU/CNRS (France), MPG (Germany) and IGN (Spain). Support for CARMA construction was derived from the Gordon and Betty Moore Foundation, the Kenneth T. and Eileen L. Norris Foundation, the James S. McDonnell Foundation, the Associates of the California Institute of Technology, the University of Chicago, the states of California, Illinois, and Maryland, and the National Science Foundation. Ongoing CARMA development and operations are supported by the National Science Foundation under a cooperative agreement, and by the CARMA partner universities. Part of the funding for GROND (both hardware as well as personnel) was generously granted from the Leibniz-Prize to Prof. G. Hasinger (DFG grant HA 1850/28-1). Funding for SDSS-III has been provided by the Alfred P. Sloan Foundation, the Participating Institutions, the National Science Foundation, and the US Department of Energy Office of Science. The SDSS-III web site is http://www.sdss3.org/. SDSSIII is managed by the Astrophysical Research Consortium for the Participating Institutions of the SDSS-III Collaboration including the University of Arizona, the Brazilian Participation Group, Brookhaven National Laboratory, Carnegie Mellon University, University of Florida, the French Participation Group, the German Participation Group, Harvard University, the Instituto de Astrofisica de Canarias, the Michigan State/Notre Dame/JINA Participation Group, Johns Hopkins University, Lawrence Berkeley National Laboratory, Max Planck Institute for Astrophysics, Max Planck Institute for Extraterrestrial Physics, New Mexico State University, New York University, Ohio State University, Pennsylvania State University, University of Portsmouth, Princeton University, the Spanish Participation Group, University of Tokyo, University of Utah, Vanderbilt University, University of Virginia, University of Washington, and Yale University.

\section{References}

Ahn, C. P., Alexandroff, R., Allende Prieto, C., et al. 2012, ApJS, 203, 21 Aihara, H., Allende Prieto, C., An, D., et al. 2011, ApJS, 193, 29

Arcavi, I., Gal-Yam, A., Yaron, O., et al. 2011, ApJ, 742, L18

Arnett, W. D. 1982, ApJ, 253, 785

Arnouts, S., Cristiani, S., Moscardini, L., et al. 1999, MNRAS, 310, 540 Asplund, M., Grevesse, N., Sauval, A. J., \& Scott, P. 2009, ARA\&A, 47, 481

Baldwin, J. A., Phillips, M. M., \& Terlevich, R. 1981, PASP, 93, 5

Barthelmy, S. D., Barbier, L. M., Cummings, J. R., et al. 2005, Space Sci. Rev., 120,143

Barthelmy, S. D., Baumgartner, W. H., Cummings, J. R., et al. 2012, GCN Circ., 13246

Baryshev, Y. V., Sokolov, I. V., Moskvitin, A. S., et al. 2010, Astrophys. Bull., 65,311

Beardmore, A. P., Evans, P. A., Goad, M. R., \& Osborne, J. P. 2012, GCN Circ., 13247

Berger, E., Chornock, R., Holmes, T. R., et al. 2011, ApJ, 743, 204 
Bertin, E., \& Arnouts, S. 1996, A\&AS, 117, 393

Beuermann, K., Hessman, F. V., Reinsch, K., et al. 1999, A\&A, 352, L26 Bhat, P. N., Briggs, M. S., Connaughton, V., et al. 2012, ApJ, 744, 141

Blinnikov, S. I., Eastman, R., Bartunov, O. S., Popolitov, V. A., \& Woosley, S. E. 1998, ApJ, 496, 454

Bloom, J. S., Kulkarni, S. R., \& Djorgovski, S. G. 2002, AJ, 123, 1111

Bostanc1, Z. F., Kaneko, Y., \& Göğüş, E. 2013, MNRAS, 428, 1623

Bromberg, O., Nakar, E., \& Piran, T. 2011, ApJ, 739, L55

Bruzual, G., \& Charlot, S. 2003, MNRAS, 344, 1000

Bufano, F., Pian, E., Sollerman, J., et al. 2012, ApJ, 753, 67

Burrows, D. N., Hill, J. E., Nousek, J. A., et al. 2005, Space Sci. Rev., 120, 165

Calzetti, D., Armus, L., Bohlin, R. C., et al. 2000, ApJ, 533, 682

Campana, S., Mangano, V., Blustin, A. J., et al. 2006, Nature, 442, 1008

Cano, Z. 2013, MNRAS, 434, 1098

Cano, Z., Bersier, D., Guidorzi, C., et al. 2011a, ApJ, 740, 41

Cano, Z., Bersier, D., Guidorzi, C., et al. 2011b, MNRAS, 413, 669

Chabrier, G., Baraffe, I., Allard, F., \& Hauschildt, P. 2000, ApJ, 542, 464

Chakraborti, S., Soderberg, A., Chomiuk, L., et al. 2014, ApJ, submitted [arXiv: 1402.6336]

Chapin, E. L., Berry, D. S., Gibb, A. G., et al. 2013, MNRAS, 430, 2545

Chapman, R., Tanvir, N. R., Priddey, R. S., \& Levan, A. J. 2007, MNRAS, 382, L21

Chatzopoulos, E., Wheeler, J. C., Vinko, J., et al. 2011, ApJ, 729, 143

Chen, H.-W. 2012, MNRAS, 419, 3039

Chevalier, R. A., \& Li, Z.-Y. 2000, ApJ, 536, 195

Chornock, R., Berger, E., Levesque, E. M., et al. 2010, unpublished [arXiv: 1004.2262]

Christensen, L., Vreeswijk, P. M., Sollerman, J., et al. 2008, A\&A, 490, 45 Christensen, L., Fynbo, J. P. U., Prochaska, J. X., et al. 2011, ApJ, 727, 73 Clocchiatti, A., Suntzeff, N. B., Covarrubias, R., \& Candia, P. 2011, AJ, 141, 163 Cobb, B. E., Bloom, J. S., Perley, D. A., et al. 2010, ApJ, 718, L150 Colgate, S. A., \& McKee, C. 1969, ApJ, 157, 623

Cucchiara, A., Levan, A., Fox, D. B., \& Tanvir, N. R. 2012, GCN Circ., 13245

De Cia, A., Starling, R. L. C., Wiersema, K., et al. 2011, MNRAS, 418, 129

de Pasquale, M., Piro, L., Gendre, B., et al. 2006, A\&A, 455, 813

de Ugarte Postigo, A., Fynbo, J. P. U., Thöne, C. C., et al. 2012a, A\&A, 548, A11

de Ugarte Postigo, A., Lundgren, A., Martín, S., et al. 2012b, A\&A, 538, A44

de Ugarte Postigo, A., Cano, Z., Thoene, C. C., et al. 2013, Central Bureau

Electronic Telegrams, 3637

Della Valle, M., Chincarini, G., Panagia, N., et al. 2006a, Nature, 444, 1050

Della Valle, M., Malesani, D., Bloom, J. S., et al. 2006b, ApJ, 642, L103

Della Valle, M., Benetti, S., Mazzali, P., et al. 2008, Central Bureau Electronic Telegrams, 1602

Dempsey, J. T., Friberg, P., Jenness, T., et al. 2013, MNRAS, 430, 2534

Dermer, C. D. 2004, ApJ, 614, 284

Detmers, R. G., Langer, N., Podsiadlowski, P., \& Izzard, R. G. 2008, A\&A, 484, 831

Dopita, M. A., Fischera, J., Sutherland, R. S., et al. 2006, ApJS, 167, 177

Ensman, L., \& Burrows, A. 1992, ApJ, 393, 742

Ergon, M., Sollerman, J., Fraser, M., et al. 2014, A\&A, 562, A17

Evans, P. A., Beardmore, A. P., Page, K. L., et al. 2007, A\&A, 469, 379

Evans, P. A., Beardmore, A. P., Page, K. L., et al. 2009, MNRAS, 397, 1177

Evans, P. A., Willingale, R., Osborne, J. P., et al. 2010, A\&A, 519, A102

Fenimore, E., \& Sumner, M. C. 1997, in All-Sky X-Ray Observations in the

Next Decade, eds. M. Matsuoka, \& N. Kawai, 167

Ferrero, P., Kann, D. A., Zeh, A., et al. 2006, A\&A, 457, 857

Ferrero, P., Klose, S., Kann, D. A., et al. 2009, A\&A, 497, 729

Filippenko, A. V. 1997, ARA\&A, 35, 309

Foley, S., Watson, D., Gorosabel, J., et al. 2006, A\&A, 447, 891

Förster Schreiber, N. M., Genzel, R., Bouché, N., et al. 2009, ApJ, 706, 1364

Friis, M., \& Watson, D. 2013, ApJ, 771, 15

Fruchter, A. S., Levan, A. J., Strolger, L., et al. 2006, Nature, 441, 463

Fryer, C. L., \& Woosley, S. E. 1998, ApJ, 502, L9

Fynbo, J. U., Holland, S., Andersen, M. I., et al. 2000, ApJ, 542, L89

Fynbo, J. P. U., Möller, P., Thomsen, B., et al. 2002, A\&A, 388, 425

Fynbo, J. P. U., Jakobsson, P., Möller, P., et al. 2003, A\&A, 406, L63

Fynbo, J. P. U., Watson, D., Thöne, C. C., et al. 2006, Nature, 444, 1047

Gal-Yam, A., Fox, D. B., Price, P. A., et al. 2006, Nature, 444, 1053

Galama, T. J., Vreeswijk, P. M., van Paradijs, J., et al. 1998, Nature, 395, 670

Gendre, B., Corsi, A., \& Piro, L. 2006, A\&A, 455, 803

Goldoni, P., Royer, F., François, P., et al. 2006, in SPIE Conf. Ser., 6269

Greiner, J., Bornemann, W., Clemens, C., et al. 2007, The Messenger, 130, 12

Greiner, J., Bornemann, W., Clemens, C., et al. 2008, PASP, 120, 405

Greiner, J., Krühler, T., McBreen, S., et al. 2009, ApJ, 693, 1912

Grupe, D., Burrows, D. N., Wu, X.-F., et al. 2010, ApJ, 711, 1008

Guetta, D., \& Della Valle, M. 2007, ApJ, 657, L73

Hamuy, M., \& Pinto, P. A. 2002, ApJ, 566, L63
Han, X. H., Hammer, F., Liang, Y. C., et al. 2010, A\&A, 514, A24

Hjorth, J. 2013, Roy. Soc. Lond. Philosophical Trans. Ser. A, 371, 20275

Hjorth, J., \& Bloom, J. S. 2012, in Gamma-Ray Bursts, eds. C. Kouveliotou,

R. A. M. J. Wijers, \& S. Woosley (Cambridge University Press), 169

Hjorth, J., Sollerman, J., Møller, P., et al. 2003, Nature, 423, 847

Hjorth, J., Malesani, D., Jakobsson, P., et al. 2012, ApJ, 756, 187

Hogg, D. W., Baldry, I. K., Blanton, M. R., \& Eisenstein, D. J. 2002, unpublished [arXiv: astro-ph/0210394]

Holland, W. S., Bintley, D., Chapin, E. L., et al. 2013, MNRAS, 430, 2513

Hook, I. M., Jørgensen, I., Allington-Smith, J. R., et al. 2004, PASP, 116, 425

Horne, K. 1986, PASP, 98, 609

Ilbert, O., Arnouts, S., McCracken, H. J., et al. 2006, A\&A, 457, 841

Jakobsson, P., Björnsson, G., Fynbo, J. P. U., et al. 2005, MNRAS, 362, 245

Jin, Z.-P., Covino, S., Della Valle, M., et al. 2013, ApJ, 774, 114

Kaneko, Y., Ramirez-Ruiz, E., Granot, J., et al. 2007, ApJ, 654, 385

Kann, D. A., Klose, S., Zhang, B., et al. 2011, ApJ, 734, 96

Kauffmann, G., Heckman, T. M., Tremonti, C., et al. 2003, MNRAS, 346, 1055

Kennicutt, Jr., R. C. 1998, ARA\&A, 36, 189

Kewley, L. J., Dopita, M. A., Sutherland, R. S., Heisler, C. A., \& Trevena, J. 2001, ApJ, 556, 121

Kewley, L. J., Geller, M. J., \& Jansen, R. A. 2004, AJ, 127, 2002

Klose, S., Nicuesa Guelbenzu, A., Kruehler, T., et al. 2013, Central Bureau

Electronic Telegrams, 3677

Kron, R. G. 1980, ApJS, 43, 305

Krühler, T., Küpcü Yoldaş, A., Greiner, J., et al. 2008, ApJ, 685, 376

Krühler, T., Greiner, J., Schady, P., et al. 2011a, A\&A, 534, A108

Krühler, T., Schady, P., Greiner, J., et al. 2011b, A\&A, 526, A153

Krühler, T., Fynbo, J. P. U., Geier, S., et al. 2012, A\&A, 546, A8

Kuin, N. P. M., \& Troja, E. 2012, GCN Circ., 13248

Kulkarni, S. R., Frail, D. A., Wieringa, M. H., et al. 1998, Nature, 395, 663

Kumar, P., \& Panaitescu, A. 2000, ApJ, 541, L51

Larson, D., Dunkley, J., Hinshaw, G., et al. 2011, ApJS, 192, 16

Laskar, T., Berger, E., \& Chary, R.-R. 2011, ApJ, 739, 1

Lazzati, D., Morsony, B. J., Blackwell, C. H., \& Begelman, M. C. 2012, ApJ, 750,68

Le Floc'h, E., Charmandaris, V., Forrest, W. J., et al. 2006, ApJ, 642, 636

Levan, A., Fruchter, A., Rhoads, J., et al. 2006, ApJ, 647, 471

Levan, A. J., Tanvir, N. R., Fruchter, A. S., et al. 2013, ApJ, submitted [arXiv: 1307.5338]

Levesque, E. M., Kewley, L. J., Berger, E., \& Zahid, H. J. 2010a, AJ, 140, 1557

Levesque, E. M., Kewley, L. J., Graham, J. F., \& Fruchter, A. S. 2010b, ApJ, 712, L26

Levesque, E. M., Berger, E., Soderberg, A. M., \& Chornock, R. 2011, ApJ, 739, 23

Levesque, E. M., Chornock, R., Soderberg, A. M., Berger, E., \& Lunnan, R. 2012, ApJ, 758, 92

Liang, E., Zhang, B., Virgili, F., \& Dai, Z. G. 2007, ApJ, 662, 111

Liang, E.-W., Yi, S.-X., Zhang, J., et al. 2010, ApJ, 725, 2209

Lyman, J. D., Bersier, D., \& James, P. A. 2014, MNRAS, 437, 3848

Maeda, K., Mazzali, P. A., Deng, J., et al. 2003, ApJ, 593, 931

Malesani, D., Tagliaferri, G., Chincarini, G., et al. 2004, ApJ, 609, L5

Malesani, D., Fynbo, J. P. U., Hjorth, J., et al. 2009, ApJ, 692, L84

Malesani, D., Schulze, S., Krühler, T., et al. 2012a, Central Bureau Electronic Telegrams, 3100

Malesani, D., Schulze, S., Krühler, T., et al. 2012b, GCN Circ., 13275

Margutti, R., Soderberg, A. M., Wieringa, M. H., et al. 2013, ApJ, 778, 18

Margutti, R., Milisavljevic, D., Soderberg, A. M., et al. 2014, ApJ, submitted [arXiv: 1402.6344]

Martin, S., Petitpas, G., \& de Ugarte Postigo, A. 2012, GCN Circ., 13260

Maselli, A., Melandri, A., Nava, L., et al. 2014, Science, 343, 48

Matheson, T., Garnavich, P. M., Stanek, K. Z., et al. 2003, ApJ, 599, 394

McBreen, S., Foley, S., Watson, D., et al. 2008, ApJ, 677, L85

Melandri, A., Pian, E., Ferrero, P., et al. 2012, A\&A, 547, A82

Mészáros, P. 2006, Rep. Prog. Phys., 69, 2259

Michałowski, M. J., Hunt, L. K., Palazzi, E., et al. 2014, A\&A, 562, A70 Milisavljevic, D., Soderberg, A. M., Margutti, R., et al. 2013, ApJ, 770, L38

Modjaz, M. 2011, Astron. Nachr., 332, 434

Modjaz, M., Li, W., Butler, N., et al. 2009, ApJ, 702, 226

Molinari, E., Vergani, S. D., Malesani, D., et al. 2007, A\&A, 469, L13

Monet, D. G., Levine, S. E., Canzian, B., et al. 2003, AJ, 125, 984

Nakar, E., \& Sari, R. 2012, ApJ, 747, 88

Nardini, M., Schmidl, S., Greiner, J., \& Kann, D. A. 2012, GCN Circ., 13256

Nousek, J. A., Kouveliotou, C., Grupe, D., et al. 2006, ApJ, 642, 389

Ofek, E. O., Cenko, S. B., Gal-Yam, A., et al. 2007, ApJ, 662, 1129

Oke, J. B., Cohen, J. G., Carr, M., et al. 1995, PASP, 107, 375

Olivares E., F., Greiner, J., Schady, P., et al. 2012, A\&A, 539, A76

Page, K. L., Starling, R. L. C., Fitzpatrick, G., et al. 2011, MNRAS, 416, 2078

Panaitescu, A., \& Kumar, P. 2000, ApJ, 543, 66 
Patat, F., Cappellaro, E., Danziger, J., et al. 2001, ApJ, 555, 900 Perley, D. A. 2012, GCN Circ., 13278

Perley, D. A., Li, W., Chornock, R., et al. 2008, ApJ, 688, 470

Perley, D. A., Morgan, A. N., Updike, A., et al. 2011, AJ, 141, 36 Perley, D. A., Cucchiara, A., Cenko, S. B., et al. 2012a, GCN Circ., 13267 Perley, D. A., Jones, T., \& Ellis, R. 2012b, GCN Circ., 13279 Perley, D. A., Levan, A. J., Tanvir, N. R., et al. 2013, ApJ, 778, 128 Perley, D. A., Cenko, S. B., Corsi, A., et al. 2014, ApJ, 781, 37 Pettini, M., \& Pagel, B. E. J. 2004, MNRAS, 348, L59

Phillips, M. M. 1993, ApJ, 413, L105

Pian, E., Mazzali, P. A., Masetti, N., et al. 2006, Nature, 442, 1011

Piranomonte, S., Ward, P. A., Fiore, F., et al. 2008, A\&A, 492, 775

Poole, T. S., Breeveld, A. A., Page, M. J., et al. 2008, MNRAS, 383, 627

Racusin, J. L., Liang, E. W., Burrows, D. N., et al. 2009, ApJ, 698, 43

Richmond, M. W., Treffers, R. R., Filippenko, A. V., et al. 1994, AJ, 107, 1022

Richmond, M. W., van Dyk, S. D., Ho, W., et al. 1996, AJ, 111, 327

Roming, P. W. A., Kennedy, T. E., Mason, K. O., et al. 2005, Space Sci. Rev., 120,95

Sánchez-Ramírez, R., Leloudas, G., Postigo, A. D. U., et al. 2012, GCN Circ., 13281

Sari, R., \& Piran, T. 1999, ApJ, 520, 641

Sari, R., Piran, T., \& Narayan, R. 1998, ApJ, 497, L17

Sari, R., Piran, T., \& Halpern, J. P. 1999, ApJ, 519, L17

Sault, R. J., Teuben, P. J., \& Wright, M. C. H. 1995, in Astronomical Data Analysis Software and Systems IV, eds. R. A. Shaw, H. E. Payne, \& J. J. E. Hayes, ASP Conf. Ser., 77, 433

Savaglio, S., Glazebrook, K., \& Le Borgne, D. 2009, ApJ, 691, 182 Schlegel, D. J., Finkbeiner, D. P., \& Davis, M. 1998, ApJ, 500, 525

Schulze, S., Klose, S., Björnsson, G., et al. 2011, A\&A, 526, A23

Schulze, S., Levan, A. J., Malesani, D., et al. 2012a, GCN Circ., 13257

Schulze, S., Malesani, D., de Ugarte Postigo, A., et al. 2012b, GCN Circ., 13252

Shen, R., \& Matzner, C. D. 2012, ApJ, 744, 36

Skrutskie, M. F., Cutri, R. M., Stiening, R., et al. 2006, AJ, 131, 1163

Smith, I. A., Tilanus, R. P. J., Tanvir, N. R., \& Frail, D. A. 2012, GCN Circ., 13259

Soderberg, A. M., Kulkarni, S. R., Nakar, E., et al. 2006a, Nature, 442, 1014

Soderberg, A. M., Nakar, E., Berger, E., \& Kulkarni, S. R. 2006b, ApJ, 638, 930

Soderberg, A. M., Berger, E., Page, K. L., et al. 2008, Nature, 453, 469

Soderberg, A. M., Chakraborti, S., Pignata, G., et al. 2010, Nature, 463, 513

Soderberg, A. M., Margutti, R., Zauderer, B. A., et al. 2012, ApJ, 752, 78

Sparre, M., \& Starling, R. L. C. 2012, MNRAS, 427, 2965

Sparre, M., Sollerman, J., Fynbo, J. P. U., et al. 2011, ApJ, 735, L24

Staley, T. D., Titterington, D. J., Fender, R. P., et al. 2013, MNRAS, 428, 3114

Stanek, K. Z., Matheson, T., Garnavich, P. M., et al. 2003, ApJ, 591, L17

Stanek, K. Z., Garnavich, P. M., Nutzman, P. A., et al. 2005, ApJ, 626, L5

Starling, R. L. C., Wiersema, K., Levan, A. J., et al. 2011, MNRAS, 411, 2792

Starling, R. L. C., Page, K. L., Pe'Er, A., Beardmore, A. P., \& Osborne, J. P. 2012, MNRAS, 427, 2950

Stritzinger, M., Hamuy, M., Suntzeff, N. B., et al. 2002, AJ, 124, 2100

Tanvir, N. R., Rol, E., Levan, A. J., et al. 2010, ApJ, 725, 625

Tanvir, N. R., Levan, A. J., Cucchiara, A., \& Fox, D. B. 2012, GCN Circ., 13251

Thöne, C. C., Fynbo, J. P. U., Östlin, G., et al. 2008, ApJ, 676, 1151

Thöne, C. C., de Ugarte Postigo, A., Fryer, C. L., et al. 2011, Nature, 480, 72

Tody, D. 1993, in Astronomical Data Analysis Software and Systems II, eds.

R. J. Hanisch, R. J. V. Brissenden, \& J. Barnes, ASP Conf. Ser., 52, 173

Troja, E., D’Elia, V., Guidorzi, C., et al. 2012, GCN Circ., 13243

Valenti, S., Benetti, S., Cappellaro, E., et al. 2008, MNRAS, 383, 1485

Valenti, S., Fraser, M., Benetti, S., et al. 2011, MNRAS, 416, 3138

van Marle, A. J., Langer, N., Achterberg, A., \& García-Segura, G. 2006, A\&A, 460,105

Vergani, S. D., Flores, H., Covino, S., et al. 2011, A\&A, 535, A127

Vernet, J., Dekker, H., D’Odorico, S., et al. 2011, A\&A, 536, A105

Virgili, F. J., Liang, E.-W., \& Zhang, B. 2009, MNRAS, 392, 91

Wanderman, D., \& Piran, T. 2010, MNRAS, 406, 1944

Wiersema, K., Savaglio, S., Vreeswijk, P. M., et al. 2007, A\&A, 464, 529

Wiersema, K., Cucchiara, A., Levan, A. J., et al. 2012, GCN Circ., 13276

Woosley, S. E. 2012, in Gamma-Ray Bursts, ed. C. Kouveliotou, R. A. M. J.

Wijers, \& S. Woosley (Cambridge University Press), 191

Woosley, S. E., \& Bloom, J. S. 2006, ARA\&A, 44, 507

Xu, D., de Ugarte Postigo, A., Leloudas, G., et al. 2013, ApJ, 776, 98

Yoldaş, A. K., Krühler, T., Greiner, J., et al. 2008, in AIP Conf. Ser. 1000, eds.

M. Galassi, D. Palmer, \& E. Fenimore, 227

Zauderer, A., Berger, E., \& Laskar, T. 2012, GCN Circ., 13254

Zeh, A., Klose, S., \& Hartmann, D. H. 2004, ApJ, 609, 952

Zeh, A., Klose, S., \& Kann, D. A. 2006, ApJ, 637, 889

Zhang, B., \& Mészáros, P. 2004, Int. J. Mod. Phys. A, 19, 2385

Zhang, B., Zhang, B.-B., Virgili, F. J., et al. 2009, ApJ, 703, 1696

Zhang, B.-B., Fan, Y.-Z., Shen, R.-F., et al. 2012, ApJ, 756, 190
${ }^{1}$ Instituto de Astrofísica, Facultad de Física, Pontificia Universidad Católica de Chile, Av. Vicuña Mackenna 4860, Santiago, Chile e-mail: sschulze@astro.puc.cl

2 Millennium Center for Supernova Science

${ }^{3}$ Centre for Astrophysics and Cosmology, Science and, University of Iceland, Dunhagi 5, 107 Reykjavík, Iceland

${ }^{4}$ Dark Cosmology Centre, Niels Bohr Institute, University of Copenhagen, Juliane Maries Vej 30, 2100 Copenhagen, Denmark

5 Department of Astronomy and Astrophysics, UCO/Lick Observatory, University of California, Santa Cruz, CA 95064, USA

${ }^{6}$ Department of Physics and Astronomy, University of Leicester, University Road, Leicester LE1 7RH, UK

${ }^{7}$ Instituto de Astrofísica de Andalucía Consejo Superior de Investigaciones Científicas (IAA-CSIC), Glorieta de la Astronomía s/n, 18008 Granada, Spain

8 The Oskar Klein Centre, Department of Physics, Stockholm University, AlbaNova University Centre, 10691 Stockholm, Sweden

9 Astrophysics Research Institute, Liverpool John Moores University, IC2, Liverpool Science Park Liverpool L3 5RF, UK

10 Department of Astronomy, California and of Technology, MC 24917, 1200 East California Blvd, Pasadena CA 91125, USA

11 Hubble Fellow

12 Max-Planck-Institut für extraterrestrische Physik, Giessenbachstrasse 1, 85748 Garching, Germany

13 Departamento de Astronomía, Universidad de Chile, Casilla 36-D, Santiago, Chile

14 Astrophysics Science Division, NASA Goddard Space Flight Center, Mail Code 661, Greenbelt MD 20771, USA

15 Department of Astronomy, University of California, Berkeley, CA 94720-3411, USA

16 Department of Particle Physics and Astrophysics, Faculty of Physics, Weizmann Institute of Science, 76100 Rehovot, Israel

17 Astronomical and Anton Pannekoek, University of Amsterdam, Science Park 904, 1098 XH Amsterdam, The Netherlands

18 Thüringer Landessternwarte Tautenburg, Sternwarte 5, 07778 Tautenburg, Germany

19 Department of Physics, University of Warwick, Coventry, CV4 7AL, UK

20 European Southern Observatory, Alonso de Córdova 3107, Vitacura Casilla 19001, Santiago 19, Chile

21 Departamento de Ciencias Fisicas, Universidad Andres Bello, Avda. Republica 252, Santiago, Chile

22 Research School of Astronomy and Astrophysics, Mount Stromlo Observatory, Cotter Road, Weston Creek, ACT 2611, Australia

23 The Oskar Klein Centre, Department of Astronomy, Stockholm University, AlbaNova University Centre, 10691 Stockholm, Sweden

24 Department of Physics and Astronomy, Rice University, 6100 South Main MS-108, Houston, TX 77005-1892, USA

25 Harvard-Smithsonian Center for Astrophysics, 60 Garden Street, Cambridge, MA 02138, USA

26 Department of Physics, Harvard University, 17 Oxford Street, Cambridge, MA 02138, USA

27 INAF - Osservatorio Astronomico di Brera, via E. Bianchi 4623807 Merate, Italy

28 Institute de Radioastronomie Millimétrique (IRAM), 300 rue de la Piscine, 38406 Saint Martin d'Hères, France

29 ASI-Science Data Center, via del Politecnico, 00133 Roma, Italy

${ }^{30}$ National Radio Astronomy Observatory, PO Box O, Socorro NM 87801, USA

31 Nordic Optical Telescope, Apartado 474, 38700 Santa Cruz de La Palma, Spain

32 APC, Astroparticule et Cosmologie, Université Paris Diderot, CNRS/IN2P3, CEA/Irfu, Observatoire de Paris, Sorbonne Paris Cité, 10 rue Alice Domon et Léonie Duquet, 75205 Paris Cedex 13, France

33 Finnish Centre for Astronomy with ESO (FINCA), University of Turku, Väisäläntie 20, 21500 Piikkiö, Finland 
34 Università degli studi di Milano-Bicocca, Piazza della Scienza 3, 20126 Milano, Italy

35 Department of Physics, University of Tokyo, 7-3-1 Hongo, Bunkyoku, 113-0033 Tokyo, Japan

36 Kavli Institute for the Physics and Mathematics of the Universe (Kavli IPMU, WPI), University of Tokyo, 277-8583 Chiba, Japan

37 Aryabhatta Research and of observational-sciences, Manora peak, Nainital 263 129, India

38 James Clerk Maxwell Telescope, Joint Astronomy Centre, 660 North A'ohoku Place, University Park, Hilo, HI 96720, USA

39 Netherlands Organization for Scientific Research, Laan van Nieuw Oost-Indie 300, 2509 AC The Hague, The Netherlands

40 Astrophysics Research Centre, School of Mathematics and Physics, Queen's University Belfast, Belfast BT7 1NN, UK
41 Department of Astronomy \& Astrophysics, The University of Chicago, 5640 S. Ellis Avenue, Chicago, IL 60637, USA

42 Kavli Institute for Cosmological Physics at the University of Chicago, USA

43 Space Science Institute, 4750 Walnut Street, Suite 205, Boulder, CO 80301, USA

44 Unidad Asociada Grupo Ciencia Planetarias UPV/EHU-IAA/CSIC, Departamento de Física Aplicada I, E.T.S. Ingeniería, Universidad del País Vasco UPV/EHU, Alameda de Urquijo s/n, 48013 Bilbao, Spain

45 Ikerbasque, Basque Foundation for Science, Alameda de Urquijo 36-5, 48008 Bilbao, Spain

46 INAF-Osservatorio Astronomico di Roma, via Frascati 33, 00040 Monteporzio Catone, Italy 
Appendix A: Photometry of the optical transient

Table A.1. Log of optical and NIR observations.

\begin{tabular}{|c|c|c|c|c|c|}
\hline $\begin{array}{l}\text { MJD } \\
\text { (days) }\end{array}$ & $\begin{array}{c}\text { Epoch } \\
\text { (s) }\end{array}$ & at & Iter & $\begin{array}{c}\text { Exposure } \\
\text { time (s) }\end{array}$ & $\begin{array}{c}\text { Brightness } \\
\left(\operatorname{mag}_{\mathrm{AB}}\right)\end{array}$ \\
\hline 56039.308 & 664.2 & Swift/UVOT & uvw2 & 38.9 & $>20$ \\
\hline 56039.313 & 1110.2 & vift/UVOT & $w 2$ & 38.9 & \\
\hline 56039.373 & 6244.8 & Swift/UVOT & $w w 2$ & 332.2 & 20.5 \\
\hline 56066.505 & 2350484 & Swift/UVOT & uvw2 & 16823.3 & $>24.05$ \\
\hline 56039.311 & 935.9 & VOT & uvm 2 & 77.8 & $\begin{array}{l}75 \\
44\end{array}$ \\
\hline 56039.370 & 5967.3 & OT & uvm 2 & 196.6 & \\
\hline 56039.436 & 11694.8 & OT & uvm 2 & 885.6 & \\
\hline 56066.471 & 2347530 & Swift/UVOT & uvm 2 & 16365 & $>23$ \\
\hline 56039.311 & 912.7 & Swift & $u v w 1$ & 58.3 & -20.00 \\
\hline 56039.364 & 5454.6 & VOT & uvw1 & 393.2 & $21.43_{-033}^{+0.48}$ \\
\hline 56039.445 & 12479.4 & Swift/UVOT & uvw1 & 645.3 & $21.56_{-0.37}^{+0.37}$ \\
\hline 56039.598 & 25671.6 & Swift/UVOT & uvw1 & 1771.2 & $21.47_{-018}^{+0.21}$ \\
\hline 56067.110 & 2402743 & Swift/UVOT & uvw1 & 11529.3 & $>23$ \\
\hline 56039.305 & 387.2 & VOT & $u$ & 245.8 & 21.06 \\
\hline 5603 & 898.9 & & , & & \\
\hline 56039.366 & 5659.9 & $S w$ & u & 393.2 & 43 \\
\hline 56039.530 & 19853.4 & & 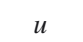 & 177 & 27 \\
\hline 56039.645 & 29800.8 & Swl & 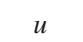 & 651.6 & 39 \\
\hline 56042.104 & 242229.9 & $S w$ & 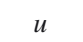 & 9 & 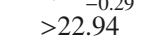 \\
\hline & 109 & Swi & $u$ & 69897.4 & 23.00 \\
\hline 56 & 325 & Swe & , & & \\
\hline 56039 & 3328.3 & Swift/UVOT & $b$ & 451.6 & $>20.93$ \\
\hline 5603 & 20641.6 & $S w$ & $b$ & 152 & 21.4 \\
\hline 5604 & 116790.9 & Swi & $b$ & 1444 & $>2$ \\
\hline 5606 & 191 & Swif & $b$ & 7250.3 & $22.05_{-0.27}^{+0.36}$ \\
\hline 5609 & 466 & Swift & $b$ & 449.7 & $>20.3$ \\
\hline & & & 0 & & \\
\hline 56 & 9 & & 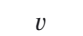 & & \\
\hline 56039.541 & 2078 & & 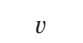 & & \\
\hline 56041.111 & & & & & \\
\hline 56093.300 & 4665526 & Swift/UVOT & 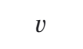 & 44 & $>19.17$ \\
\hline 560 & 5 & & $g^{\prime}$ & 15 & 22.2 \\
\hline 5604 & 61 & & $g^{\prime}$ & $4 \times 369$ & $22.12 \pm 0.05$ \\
\hline 56040.036 & 63556 & GROND & $g^{\prime}$ & $4 \times 369$ & $22.15 \pm 0.07$ \\
\hline 56040.040 & 63883 & Gemini/GMC & $g^{\prime}$ & $1 \times 60$ & $22.22 \pm 0.06$ \\
\hline 56040.057 & 65383 & GROND & $g^{\prime}$ & $4 \times 369$ & $22.11 \pm 0.08$ \\
\hline 56040.089 & 68118 & GROND & $g^{\prime}$ & $8 \times 369$ & $22.18 \pm 0.06$ \\
\hline 56040.173 & 75361 & P60 & $g^{\prime}$ & 900 & $22.14 \pm 0.10$ \\
\hline 56041.067 & 152661 & $\mathrm{PO}$ & $g^{\prime}$ & $16 \times 115$ & $22.59 \pm 0.10$ \\
\hline 56041.104 & 155811 & GRO & $g^{\prime}$ & $16 \times 115$ & $22.79 \pm 0.16$ \\
\hline 56043.239 & 340286 & emini/GMO & $g^{\prime}$ & $1 \times 100$ & $22.86 \pm$ \\
\hline 56048.018 & 753173 & GROND & $g^{\prime}$ & $8 \times 369$ & $22.38 \pm 0.10$ \\
\hline 56050.011 & 925358 & GROND & $g^{\prime}$ & $8 \times 369$ & $22.35 \pm 0.08$ \\
\hline 56053.969 & 1267371 & GTC/OSIRI & $g^{\prime}$ & $1 \times 100$ & $22.14 \pm 0.11$ \\
\hline 56054.249 & 1291574 & Gemini/GMOS & $g^{\prime}$ & $1 \times 30$ & $22.16 \pm 0.07$ \\
\hline 56059.005 & 1702443 & GROND & $g^{\prime}$ & $8 \times 369$ & $22.22 \pm 0.04$ \\
\hline 56059.020 & 1703734 & Gemini/GMC & $g^{\prime}$ & $1 \times 120$ & $22.19 \pm 0.07$ \\
\hline 56067.938 & 2474262 & GROND & $g^{\prime}$ & $4 \times 369$ & $22.82 \pm 0.05$ \\
\hline
\end{tabular}

Notes. Magnitudes are corrected for Galactic extinction $(E(B-V)=$ $0.03 \mathrm{mag}$ ). Column "Epoch" shows the logarithmic mean-time after the GRB in the observer frame. We only display the total observing time of the Swift/UVOT and P60 data (see Sect. 2.2 for details). As described in Sect. 2.3, photometry was tied to the SDSS DR8 standard $\left(g^{\prime} r^{\prime} i^{\prime} z^{\prime}\right)$ and to the 2MASS standard $\left(J H K_{\mathrm{S}}\right)$. For those filters not covered by our primary calibration systems $\left(R I_{C} i Y\right)$, we used the instrument-specific band passes to transform magnitudes into the respective filter system. (a) The image is a stack of images with different exposure times. The shown time is the sum of the single images.
Table A.1. continued.

\begin{tabular}{|c|c|c|c|c|c|}
\hline $\begin{array}{l}\text { MJD } \\
\text { (days) }\end{array}$ & $\begin{array}{l}\text { Epoch } \\
\text { (s) }\end{array}$ & Instrument & Filter & $\begin{array}{c}\text { Exposure } \\
\text { time (s) }\end{array}$ & $\begin{array}{c}\text { Brightness } \\
\left(\operatorname{mag}_{\mathrm{AB}}\right)\end{array}$ \\
\hline 56078.217 & 3362396 & GROND & $g^{\prime}$ & $24 \times 115$ & $23.29 \pm 0.20$ \\
\hline 56039.414 & 9793 & Gemini/GMOS & $r^{\prime}$ & $1 \times 60$ & $21.11 \pm 0.04$ \\
\hline 56039.990 & 59563 & GROND & $r^{\prime}$ & $4 \times 115$ & $22.19 \pm 0.14$ \\
\hline 56040.015 & 61702 & GROND & $r^{\prime}$ & $4 \times 369$ & $22.17 \pm 0.07$ \\
\hline 56040.036 & 63556 & GROND & $r^{\prime}$ & $4 \times 369$ & $22.02 \pm 0.05$ \\
\hline 56040.047 & 64459 & Gemini/GMOS & $r^{\prime}$ & $1 \times 60$ & $22.10 \pm 0.05$ \\
\hline 56040.057 & 65383 & GROND & $r^{\prime}$ & $4 \times 369$ & $22.22 \pm 0.07$ \\
\hline 56040.089 & 68118 & GROND & $r^{\prime}$ & $8 \times 369$ & $22.12 \pm 0.08$ \\
\hline 56040.161 & 74357 & P60 & $r^{\prime}$ & 900 & $22.28 \pm 0.10$ \\
\hline 56040.888 & 137134 & NOT/MOSCA & $r^{\prime}$ & $4 \times 300$ & $22.28 \pm 0.08$ \\
\hline 56041.068 & 152661 & GROND & $r^{\prime}$ & $16 \times 115$ & $22.34 \pm 0.09$ \\
\hline 56041.104 & 155811 & GROND & $r^{\prime}$ & $16 \times 115$ & $22.47 \pm 0.12$ \\
\hline 56041.949 & 228856 & NOT/MOSCA & $r^{\prime}$ & $12 \times 300$ & $22.23 \pm 0.06$ \\
\hline 56042.938 & 314237 & NOT/ALFOSC & $r^{\prime}$ & $24 \times 150$ & $22.07 \pm 0.06$ \\
\hline 56043.247 & 340990 & Gemini/GMOS & $r^{\prime}$ & $1 \times 100$ & $22.12 \pm 0.06$ \\
\hline 56048.018 & 753173 & GROND & $r^{\prime}$ & $8 \times 369$ & $21.48 \pm 0.05$ \\
\hline 56048.967 & 835203 & JOT/StanCAM & $R$ & $8 \times 150$ & $21.45 \pm 0.10$ \\
\hline 56050.011 & 925358 & GROND & $r^{\prime}$ & $8 \times 369$ & $21.33 \pm 0.04$ \\
\hline 56052.958 & 1179975 & Gemini/GMOS & $r^{\prime}$ & $1 \times 30$ & $21.41 \pm 0.10$ \\
\hline 56053.886 & 1260196 & NOT/StanCAM & $R$ & $12 \times 150$ & $21.25 \pm 0.06$ \\
\hline 56053.972 & 1267581 & GTC/OSIRIS & $r^{\prime}$ & $1 \times 100$ & $21.37 \pm 0.14$ \\
\hline 56054.258 & 1292332 & Gemini/GMOS & $r^{\prime}$ & $1 \times 30$ & $21.26 \pm 0.05$ \\
\hline 56056.895 & 1520199 & NOT/ALFOSC & $r^{\prime}$ & $8 \times 150$ & $21.23 \pm 0.07$ \\
\hline 56059.032 & 1704797 & Gemini/GMOS & $r^{\prime}$ & $1 \times 120$ & $21.27 \pm 0.05$ \\
\hline 56059.005 & 1702443 & GROND & $r^{\prime}$ & $8 \times 369$ & $21.21 \pm 0.04$ \\
\hline 56061.962 & 1957915 & Gemini/GMOS & $r^{\prime}$ & $1 \times 30$ & $21.32 \pm 0.06$ \\
\hline 56063.912 & 2126451 & NOT/ALFOSC & $r^{\prime}$ & $15 \times 90$ & $21.36 \pm 0.08$ \\
\hline 56065.897 & 2297894 & NOT/MOSCA & $r^{\prime}$ & $16 \times 90$ & $21.48 \pm 0.05$ \\
\hline 56065.968 & 2304100 & Gemini/GMOS & $r^{\prime}$ & $1 \times 100$ & $21.51 \pm 0.04$ \\
\hline 56066.028 & 2309230 & Magellan/LDSS3 & $r^{\prime}$ & $3 \times 180$ & $21.55 \pm 0.04$ \\
\hline 56067.938 & 2474262 & GROND & $r^{\prime}$ & $4 \times 369$ & $21.65 \pm 0.04$ \\
\hline 56067.957 & 2475887 & NOT/MOSCA & $r^{\prime}$ & $15 \times 90$ & $21.54 \pm 0.08$ \\
\hline 56067.979 & 2477779 & DuPont/CCD & $r^{\prime}$ & $4 \times 500$ & $21.58 \pm 0.05$ \\
\hline 56069.966 & 2649489 & Gemini/GMOS & $r^{\prime}$ & $1 \times 100$ & $21.73 \pm 0.04$ \\
\hline 56069.902 & 2643961 & NOT/MOSCA & $r^{\prime}$ & $15 \times 90$ & $21.79 \pm 0.07$ \\
\hline 56070.957 & 2735091 & Gemini/GMOS & $r^{\prime}$ & $1 \times 100$ & $21.77 \pm 0.08$ \\
\hline 56071.907 & 2817199 & NOT/ALFOSC & $r^{\prime}$ & $10 \times 90$ & $21.96 \pm 0.07$ \\
\hline 56078.217 & 3362396 & GROND & $r^{\prime}$ & $24 \times 115$ & $22.43 \pm 0.11$ \\
\hline 56079.955 & 3512502 & Gemini/GMOS & $r^{\prime}$ & $1 \times 30$ & $22.41 \pm 0.11$ \\
\hline 56083.915 & 3854715 & NOT/ALFOSC & $r^{\prime}$ & $20 \times 90$ & $22.39 \pm 0.47$ \\
\hline 56039 & 1880 & Gemini/GMOS & $i^{\prime}$ & $1 \times 240$ & $20.93 \pm 0.04$ \\
\hline 56039.896 & 51451 & NOT/MOSCA & $I$ & $12 \times 300$ & $22.19 \pm 0.08$ \\
\hline 56039.990 & 59563 & GROND & $i^{\prime}$ & $4 \times 115$ & $22.01 \pm 0.18$ \\
\hline 56040.015 & 61702 & GROND & $i^{\prime}$ & $4 \times 369$ & $22.13 \pm 0.07$ \\
\hline 56040.036 & 63556 & GROND & $i^{\prime}$ & $4 \times 369$ & $22.27 \pm 0.10$ \\
\hline 56040.047 & 64459 & Gemini/GMOS & $i^{\prime}$ & $1 \times 60$ & $22.16 \pm 0.06$ \\
\hline 56040.078 & 67209 & GROND & $i^{\prime}$ & $12 \times 369$ & $22.32 \pm 0.09$ \\
\hline 56040.149 & 73289 & P60 & $i^{\prime}$ & 900 & $22.23 \pm 0.17$ \\
\hline 56040.871 & 135683 & NOT/MOSCA & $i^{\prime}$ & $4 \times 300$ & $22.48 \pm 0.12$ \\
\hline 56041.067 & 152661 & GROND & $i^{\prime}$ & $16 \times 115$ & $22.50 \pm 0.16$ \\
\hline 56041.104 & 155811 & GROND & $i^{\prime}$ & $16 \times 115$ & $22.59 \pm 0.19$ \\
\hline 56041.924 & 226705 & NOT/MOSCA & $i^{\prime}$ & $6 \times 300$ & $22.37 \pm 0.13$ \\
\hline 56042.866 & 308045 & GTC/OSIRIS & $i^{\prime}$ & $1 \times 10$ & $22.33 \pm 0.14$ \\
\hline 56042.885 & 309735 & NOT/ALFOSC & $i^{\prime}$ & $13 \times 300$ & $22.35 \pm 0.06$ \\
\hline 56043.255 & 341689 & Gemini/GMOS & $i^{\prime}$ & $1 \times 100$ & $22.29 \pm 0.06$ \\
\hline 56043.874 & 395133 & NOT/ALFOSC & $i^{\prime}$ & $12 \times 150$ & $22.18 \pm 0.08$ \\
\hline 56047.890 & 742159 & NOT/ALFOSC & $i^{\prime}$ & $3150^{a}$ & $21.56 \pm 0.08$ \\
\hline 56048.018 & 753173 & GROND & $i^{\prime}$ & $8 \times 369$ & $21.56 \pm 0.08$ \\
\hline 56048.987 & 836905 & NOT/StanCam & $i_{i}$ & $8 \times 150$ & $21.52 \pm 0.08$ \\
\hline 56050.011 & 925358 & GROND & $i^{\prime}$ & $8 \times 369$ & $21.49 \pm 0.05$ \\
\hline 56053.916 & 1262736 & NOT/StanCAM & $i_{i}$ & $12 \times 150$ & $21.34 \pm 0.05$ \\
\hline 56053.903 & 1261613 & GTC/OSIRIS & $i^{\prime}$ & $1 \times 100$ & $21.20 \pm 0.09$ \\
\hline 56063.974 & 1267805 & GTC/OSIRIS & $i^{\prime}$ & $1 \times 100$ & $21.27 \pm 0.06$ \\
\hline 56054.163 & 1284132 & P60 & $i^{\prime}$ & 1800 & $21.21 \pm 0.14$ \\
\hline 56054.264 & 1292809 & Gemini/GM & & & $21.29 \pm 0.05$ \\
\hline 56055.175 & 1371560 & P60 & $i^{\prime}$ & 3600 & $21.26 \pm 0.11$ \\
\hline 56055.884 & 1432840 & NOT/StanCAM & $i_{i}$ & $8 \times 150$ & $21.35 \pm 0.05$ \\
\hline 56058.889 & 1692472 & NOT/ALFOSC & $i^{\prime}$ & $11 \times 150$ & $21.32 \pm 0.07$ \\
\hline 56059.005 & 1702443 & GROND & $i^{\prime}$ & $8 \times 369$ & $21.27 \pm 0.03$ \\
\hline 56059.005 & 1702494 & Gemini/GMOS & $i^{\prime}$ & $1 \times 120$ & $21.24 \pm 0.04$ \\
\hline 56061.176 & 1890072 & $\mathrm{P} 60$ & $i^{\prime}$ & 3240 & $21.22 \pm 0.14$ \\
\hline 56062.181 & 1976858 & $\mathrm{P} 60$ & $i^{\prime}$ & 3600 & $21.27 \pm 0.15$ \\
\hline
\end{tabular}


Table A.1. continued.

\begin{tabular}{|c|c|c|c|c|c|}
\hline $\begin{array}{c}\text { MJD } \\
\text { (days) }\end{array}$ & $\begin{array}{c}\text { Epoch } \\
(\mathrm{s})\end{array}$ & Instrument & Filter & $\begin{array}{c}\text { Exposure } \\
\text { time (s) }\end{array}$ & $\begin{array}{c}\text { Brightness } \\
\left(\operatorname{mag}_{\mathrm{AB}}\right)\end{array}$ \\
\hline 56062.886 & 2037796 & NOT/ALFOSC & $l$ & $15 \times 90$ & $21.46 \pm 0.22$ \\
\hline 56063.180 & 2063213 & P60 & $i^{\prime}$ & 3600 & $21.33 \pm 0.10$ \\
\hline 56063.891 & 2124602 & NOT/ALFOSC & $i^{\prime}$ & $10 \times 120$ & $21.41 \pm 0.17$ \\
\hline 56065.921 & 2300003 & NOT/MOSCA & $i^{\prime}$ & $10 \times 120$ & $21.29 \pm 0.05$ \\
\hline 56066.040 & 2310267 & Magellan/LDSS3 & $i^{\prime}$ & $3 \times 180$ & $21.33 \pm 0.04$ \\
\hline 56067.934 & 2473900 & NOT/MOSCA & $i^{\prime}$ & $10 \times 120$ & $21.35 \pm 0.05$ \\
\hline 56067.938 & 2474262 & GROND & $i^{\prime}$ & $4 \times 369$ & $21.47 \pm 0.06$ \\
\hline 56068.014 & 2480838 & DuPont/CCD & $i^{\prime}$ & $4 \times 500$ & $21.41 \pm 0.04$ \\
\hline 56068.923 & 2559358 & NOT/MOSCA & $i^{\prime}$ & $19 \times 60$ & $21.39 \pm 0.06$ \\
\hline 56069.1911 & 2582542 & $\mathrm{P} 60$ & $i^{\prime}$ & 720 & $21.50 \pm 0.09$ \\
\hline 56070.0313 & 2655131 & Magellan/LDSS3 & $i^{\prime}$ & $3 \times 300$ & $21.40 \pm 0.06$ \\
\hline 56070.1766 & 2667689 & P60 & $i^{\prime}$ & 2340 & $21.59 \pm 0.15$ \\
\hline 56070.9694 & 2736187 & Gemini/GMOS & $i^{\prime}$ & $1 \times 120$ & $21.62 \pm 0.04$ \\
\hline 56071.8946 & 2816120 & NOT/ALFOSC & $i^{\prime}$ & $13 \times 90$ & $21.63 \pm 0.08$ \\
\hline 56076.8994 & 3248536 & NOT/ALFOSC & $i^{\prime}$ & $20 \times 90$ & $21.98 \pm 0.09$ \\
\hline 56078.2172 & 3362396 & GROND & $i^{\prime}$ & $24 \times 115$ & $22.06 \pm 0.12$ \\
\hline 56079.8939 & 3507261 & NOT/ALFOSC & $i^{\prime}$ & $30 \times 90$ & $22.08 \pm 0.11$ \\
\hline 56083.8841 & 3852020 & NOT/ALFOSC & $i^{\prime}$ & $20 \times 90$ & $22.15 \pm 0.21$ \\
\hline 56039.9900 & 59563 & GROND & $z^{\prime}$ & $4 \times 115$ & $22.01 \pm 0.20$ \\
\hline 56040.0360 & 63541 & GROND & $z^{\prime}$ & $12 \times 369$ & $22.09 \pm 0.11$ \\
\hline 56040.0599 & 65605 & Gemini/GMOS & $z^{\prime}$ & $1 \times 60$ & $22.16 \pm 0.11$ \\
\hline 56040.0890 & 68118 & GROND & $z^{\prime}$ & $8 \times 369$ & $22.37 \pm 0.17$ \\
\hline 56041.0675 & 152661 & GROND & $z^{\prime}$ & $8 \times 369$ & $22.17 \pm 0.16$ \\
\hline 56041.1039 & 155811 & GROND & $z^{\prime}$ & $8 \times 369$ & $22.28 \pm 0.27$ \\
\hline 56043.2633 & 342383 & Gemini/GMOS & $z^{\prime}$ & $1 \times 100$ & $22.59 \pm 0.07$ \\
\hline 56048.0179 & 753173 & GROND & $z^{\prime}$ & $8 \times 369$ & $21.86 \pm 0.15$ \\
\hline 56049.8781 & 913895 & CAHA/Omega 2000 & $z^{\prime}$ & $20 \times 90$ & $21.93 \pm 0.08$ \\
\hline 56050.0107 & 925358 & GROND & $z^{\prime}$ & $8 \times 369$ & $21.78 \pm 0.12$ \\
\hline 56052.2596 & 1119664 & UKIRT/WFCAM & $z^{\prime}$ & $4 \times 360$ & $21.44 \pm 0.13$ \\
\hline 56053.9770 & 1268047 & GTC/OSIRIS & $z^{\prime}$ & $3 \times 70$ & $21.58 \pm 0.07$ \\
\hline 56054.2708 & 1293429 & Gemini/GMOS & $z^{\prime}$ & $1 \times 30$ & $21.63 \pm 0.06$ \\
\hline 56058.9733 & 1699722 & Gemini/GMOS & $z^{\prime}$ & $1 \times 120$ & $21.48 \pm 0.04$ \\
\hline 56059.0048 & 1702443 & GROND & $z^{\prime}$ & $8 \times 369$ & $21.48 \pm 0.07$ \\
\hline 56067.9101 & 2471861 & NOT/MOSCA & $z^{\prime}$ & $12 \times 120$ & $21.74 \pm 0.15$ \\
\hline 56067.9379 & 2474262 & GROND & $z^{\prime}$ & $4 \times 369$ & $21.62 \pm 0.07$ \\
\hline 56068.8970 & 2557129 & NOT/MOSCA & $z^{\prime}$ & $13 \times 120$ & $21.59 \pm 0.12$ \\
\hline 56070.9776 & 2736894 & Gemini/GMOS & $z^{\prime}$ & $1 \times 120$ & $21.86 \pm 0.06$ \\
\hline 56078.2172 & 3362396 & GROND & $z^{\prime}$ & $24 \times 115$ & $21.81 \pm 0.12$ \\
\hline 56049.9204 & $91^{\prime}$ & CAHA/Omega 2000 & $Y$ & $20 \times 90$ & $21.93 \pm 0.17$ \\
\hline 56039.3537 & 4589 & UKII & $J$ & 360 & 20. \\
\hline 56039.3590 & 5048 & UKIRT/WFCAM & $J$ & 360 & $20.33 \pm 0.07$ \\
\hline 56039.3644 & 5514 & UKIRT/WFCAM & $J$ & 360 & $20.36 \pm 0.07$ \\
\hline 56039.3698 & 5979 & UKIRT/WFCAM & $J$ & 360 & $20.41 \pm 0.07$ \\
\hline 56048.9026 & 829616 & CAHA/Omega 2000 & $J$ & $60 \times 60$ & $21.60 \pm 0.17$ \\
\hline 56049.9727 & 922069 & CAHA/Omega 2000 & $J$ & $30 \times 60$ & $21.83 \pm 0.20$ \\
\hline 56052.2354 & 1117569 & UKIRT/WFCAM & $J$ & $4 \times 360$ & $21.75 \pm 0.24$ \\
\hline 56054.2834 & 1294514 & Gemini-N/NIRI & $J$ & $1 \times 60$ & $21.96 \pm 0.11$ \\
\hline 56065.2816 & 2244761 & UKIRT/WFCAM & $J$ & $6 \times 360$ & $21.90 \pm 0.15$ \\
\hline 56049.1898 & 854426 & P200/WIRC & $J$ & $15 \times 240$ & $21.68 \pm 0.16$ \\
\hline 56039.3757 & 6493 & UKIRT/WFCAM & $H$ & 360 & $20.29 \pm 0.09$ \\
\hline 56039.3812 & 6963 & UKIRT/WFCAM & $H$ & 360 & $20.32 \pm 0.09$ \\
\hline 56039.3866 & 7432 & UKIRT/WFCAM & $H$ & 360 & $20.51 \pm 0.11$ \\
\hline 56039.3919 & 7894 & UKIRT/WFCAM & $H$ & 360 & $20.34 \pm 0.10$ \\
\hline 56040.3685 & 92266 & UKIRT/WFCAM & $H$ & $4 \times 360$ & $21.65 \pm 0.42$ \\
\hline 56042.3348 & 262155 & UKIRT/WFCAM & $H$ & $4 \times 360$ & $22.29 \pm 0.31$ \\
\hline 56054.2376 & 1290555 & Gemini-N/NIRI & $K$ & $1 \times 60$ & $21.46 \pm 0.14$ \\
\hline
\end{tabular}

\section{Appendix B: Late time observations}

Table B.1. Summary of late-time observations.

\begin{tabular}{|c|c|c|c|c|}
\hline $\begin{array}{c}\text { MJD } \\
\text { (days) }\end{array}$ & $\begin{array}{c}\text { Epoch } \\
\text { (s) }\end{array}$ & Instrument & Filter & $\begin{array}{c}\text { Exposure } \\
\text { time (s) }\end{array}$ \\
\hline 56205.1849 & 14332405 & CAHA/BUSCA & $u^{\prime}$ & $13 \times 45$ \\
\hline 56206.1974 & 14419882 & CAHA/BUSCA & $u^{\prime}$ & $50 \times 45$ \\
\hline 56208.1930 & 14592304 & CAHA/BUSCA & $u^{\prime}$ & $21 \times 45$ \\
\hline 56209.1754 & 14677188 & CAHA/BUSCA & $u^{\prime}$ & $52 \times 45$ \\
\hline 56205.1849 & 14332405 & CAHA/BUSCA & $g^{\prime}$ & $13 \times 45$ \\
\hline 56206.1974 & 14419882 & CAHA/BUSCA & $g^{\prime}$ & $50 \times 45$ \\
\hline 56208.1930 & 14592304 & CAHA/BUSCA & $g^{\prime}$ & $21 \times 45$ \\
\hline 56209.1754 & 14677188 & CAHA/BUSCA & $g^{\prime}$ & $52 \times 45$ \\
\hline 56205.1849 & 14332405 & CAHA/BUSCA & $r^{\prime}$ & $13 \times 45$ \\
\hline 56206.1974 & 14419882 & CAHA/BUSCA & $r^{\prime}$ & $50 \times 45$ \\
\hline 56208.1930 & 14592304 & CAHA/BUSCA & $r^{\prime}$ & $21 \times 45$ \\
\hline 56209.1754 & 14677188 & CAHA/BUSCA & $r^{\prime}$ & $52 \times 45$ \\
\hline 56205.1849 & 14332405 & CAHA/BUSCA & $z^{\prime}$ & $13 \times 45$ \\
\hline 56206.1974 & 14419882 & CAHA/BUSCA & $z^{\prime}$ & $50 \times 45$ \\
\hline 56208.1930 & 14592304 & CAHA/BUSCA & $z^{\prime}$ & $21 \times 45$ \\
\hline 56209.1754 & 14677188 & CAHA/BUSCA & $z^{\prime}$ & $52 \times 45$ \\
\hline 56245.1818 & 17788140 & LT/IO:O & $r^{\prime}$ & $5 \times 100$ \\
\hline 56254.1677 & 18564517 & LT/IO:O & $r^{\prime}$ & $9 \times 100$ \\
\hline 56270.1558 & 19945888 & LT/IO:O & $r^{\prime}$ & $9 \times 100$ \\
\hline 56275.1809 & 20380060 & LT/IO:O & $r^{\prime}$ & $9 \times 100$ \\
\hline 56277.2116 & 20555512 & LT/IO:O & $r^{\prime}$ & $9 \times 100$ \\
\hline 56279.0852 & 20717394 & LT/IO:O & $r^{\prime}$ & $9 \times 100$ \\
\hline 56282.2041 & 20986865 & LT/IO:O & $r^{\prime}$ & $9 \times 100$ \\
\hline 56283.0932 & 21063685 & LT/IO:O & $r^{\prime}$ & $9 \times 100$ \\
\hline 56284.1360 & 21153781 & LT/IO:O & $r^{\prime}$ & $9 \times 100$ \\
\hline 56296.0438 & 22182616 & LT/IO:O & $r^{\prime}$ & $9 \times 100$ \\
\hline 56300.9702 & 22608259 & LT/IO:O & $r^{\prime}$ & $9 \times 100$ \\
\hline 56302.1352 & 22708908 & LT/IO:O & $r^{\prime}$ & $9 \times 100$ \\
\hline 56303.1084 & 22792994 & LT/IO:O & $r^{\prime}$ & $9 \times 100$ \\
\hline 56303.9884 & 22869026 & LT/IO:O & $r^{\prime}$ & $9 \times 100$ \\
\hline 56305.0037 & 22956752 & LT/IO:O & $r^{\prime}$ & $9 \times 100$ \\
\hline 56306.0300 & 23045418 & LT/IO:O & $r^{\prime}$ & $9 \times 100$ \\
\hline 56310.0862 & 23395876 & LT/IO:O & $r^{\prime}$ & $15 \times 100$ \\
\hline 56310.9812 & 23473205 & LT/IO:O & $r^{\prime}$ & $15 \times 100$ \\
\hline 56312.1042 & 23570230 & LT/IO:O & $r^{\prime}$ & $15 \times 100$ \\
\hline 56360.9795 & 27793059 & LT/IO:O & $r^{\prime}$ & $15 \times 100$ \\
\hline 56364.8835 & 28130364 & LT/IO:O & $r^{\prime}$ & $15 \times 100$ \\
\hline 56365.9379 & 28221466 & LT/IO:O & $r^{\prime}$ & $15 \times 100$ \\
\hline 56370.9299 & 28652774 & LT/IO:O & $r^{\prime}$ & $15 \times 100$ \\
\hline 56309.4711 & 23342731 & Gemini-N/GMOS & $g^{\prime}$ & $5 \times 100$ \\
\hline 56309.4704 & 23342671 & Gemini-N/GMOS & $r^{\prime}$ & $5 \times 100$ \\
\hline 56309.4624 & 23341981 & Gemini-N/GMOS & $i^{\prime}$ & $5 \times 100$ \\
\hline
\end{tabular}

Notes. Column "Epoch" shows the logarithmic mean-time after the burst in the observer frame. 


\section{Appendix C: Afterglow modelling}

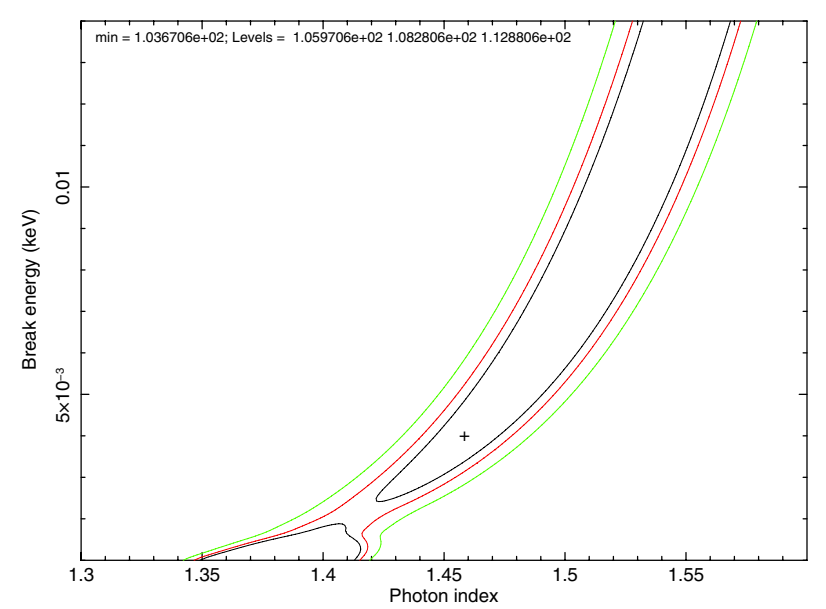

Fig. C.1. Contour plot of the best-fit parameters: the photon index $\Gamma$, defined as $\Gamma=\beta_{0}+1$, and the break energy. The contours correspond to regions of $68 \%, 90 \%$, and $99 \%$ confidence level for two parameters. The $\chi^{2}$ values for the different levels are shown in the figure.

\section{Appendix D: Spectra and SEDs of selected galaxies}
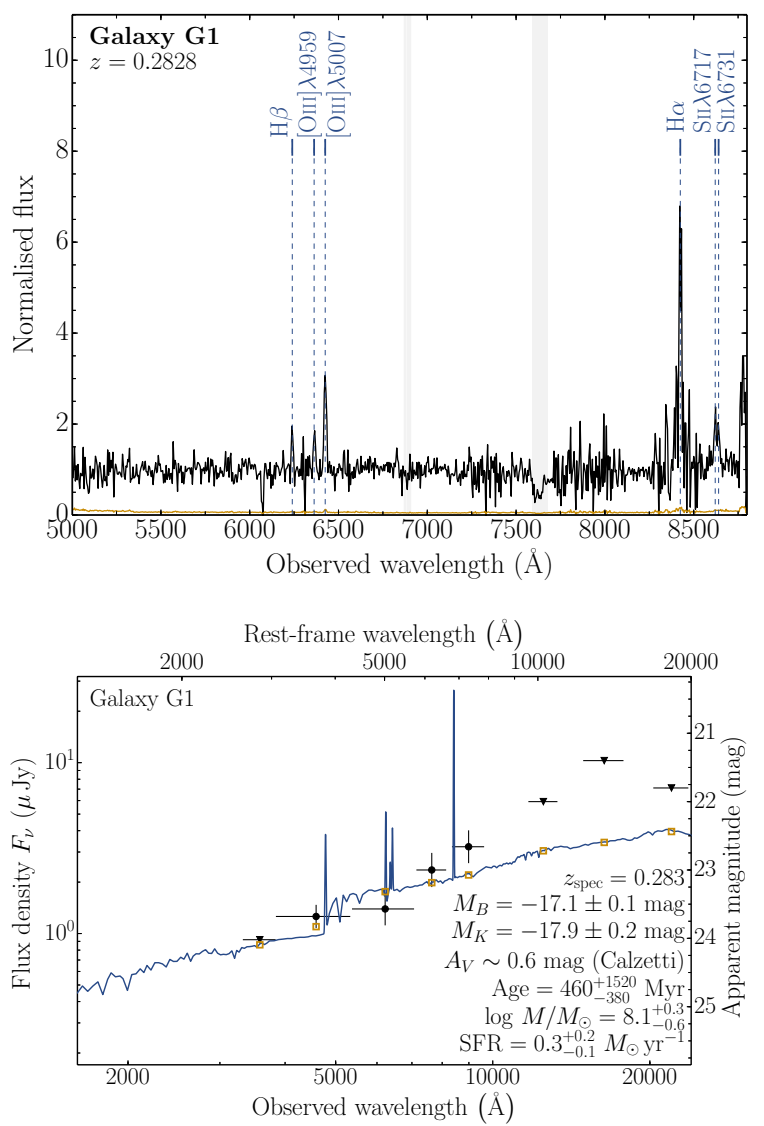

Fig. D.1. Top: normalised spectrum of galaxy G1 (Fig. 9; Table 6) obtained with GTC/OSIRIS 3.6 days after the GRB. Several emission lines are detected at $z=0.2828$. $\mathrm{H} \alpha$ is partly blended with a sky emission line. The error spectrum is shown in orange. The positions of telluric bands are highlighted by grey-shaded areas. Bottom: spectral energy distribution from 1600 to $24000 \AA$ (similar to Fig. 8). The solid line displays the best-fit model obtained with Le Phare $\left(\chi^{2}=3.1\right.$, number of filters $=4)$. The beige open squares represent the model predicted magnitudes. Upper limits are displayed as triangles.

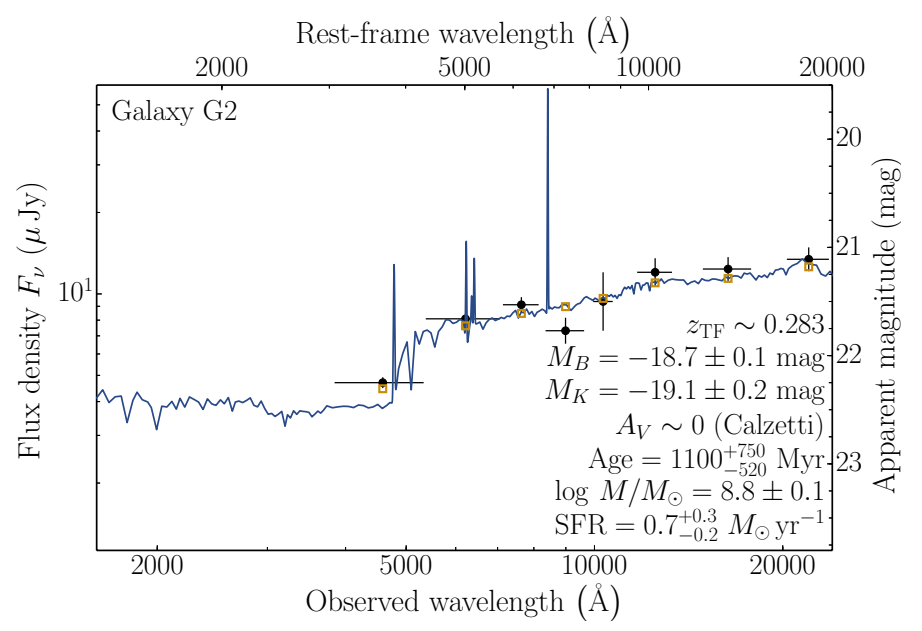

Fig. D.2. Same as Fig. D.1 but for galaxy G2 (Fig. 9; Table 6). The fit statistics are $\chi^{2}=14.8$ for eight filters. The redshift was set to $z=0.283$ from the tuneable filters.

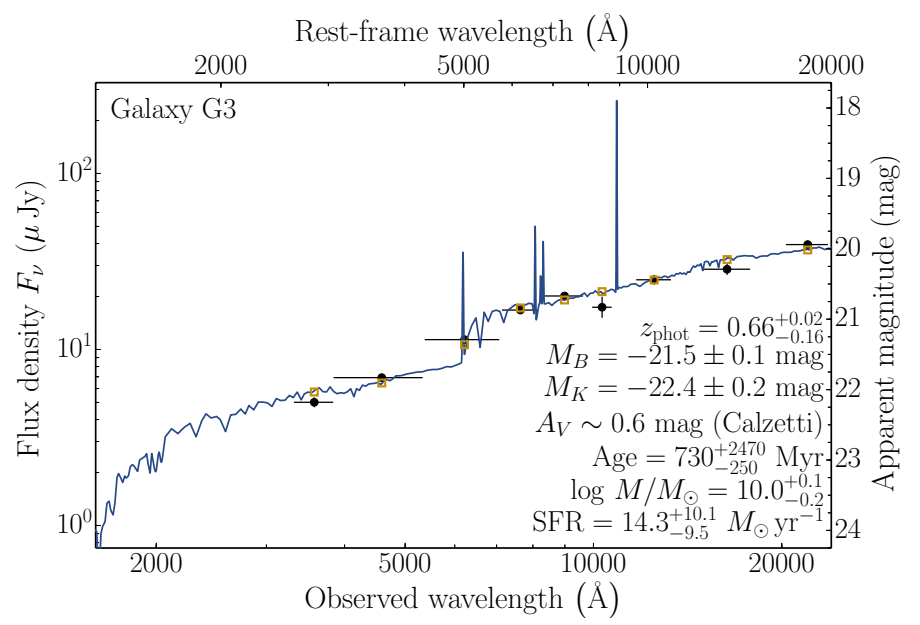

Fig. D.3. Same as Fig. D.1 but for galaxy G3 (Table 6). The fit statistics are $\chi^{2}=18$ for nine filters. 


\section{Appendix E: X-shooter spectra of the afterglow and host galaxy's nucleus}

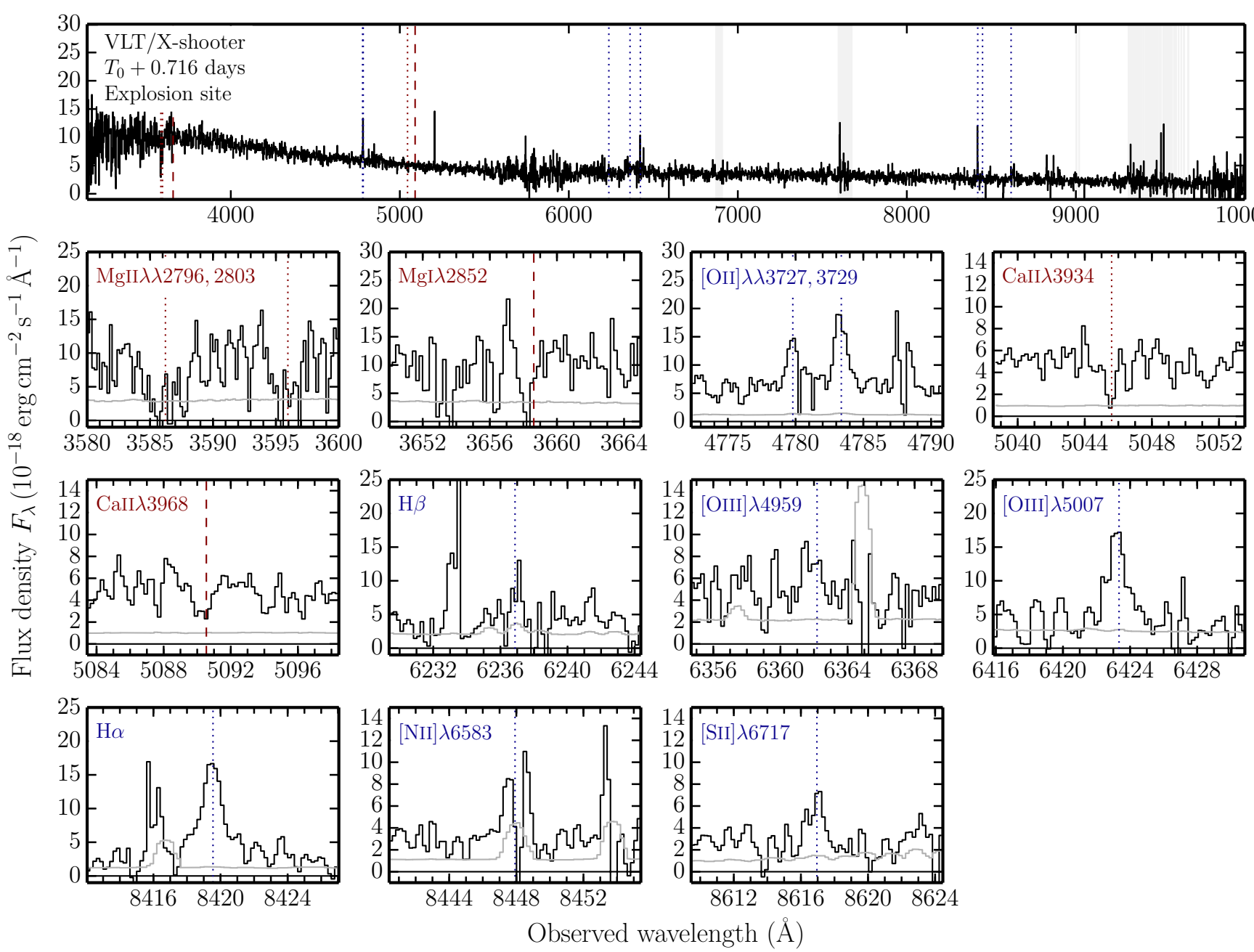

Fig. E.1. X-shooter spectrum of GRB 120422A's afterglow obtained 0.716 days after the burst. The top panel shows the combined UVB- and VIS-arm spectrum from 3150 to $10000 \AA$. The absolute flux-calibrated spectrum is corrected for heliocentric motion and Galactic reddening. The spectral data are shown in black, and the corresponding noise level in grey. For illustrative purposes, we rebinned the spectrum to $2 \AA$ bins. The positions of absorption lines that are typically associated with GRB absorbers are indicated by red lines (dotted if detected and dashed if a feature evaded detection). Nebular lines are shown in blue. The panels below zoom-in on each absorption and emission line (wavelength binning $0.15 \AA$ ). Table 4 summarises the fluxes and equivalent widths for each line. Regions that are heavily affected by atmospheric absorption (transparency: $<20 \%$ ) are indicated by the grey shaded areas. 


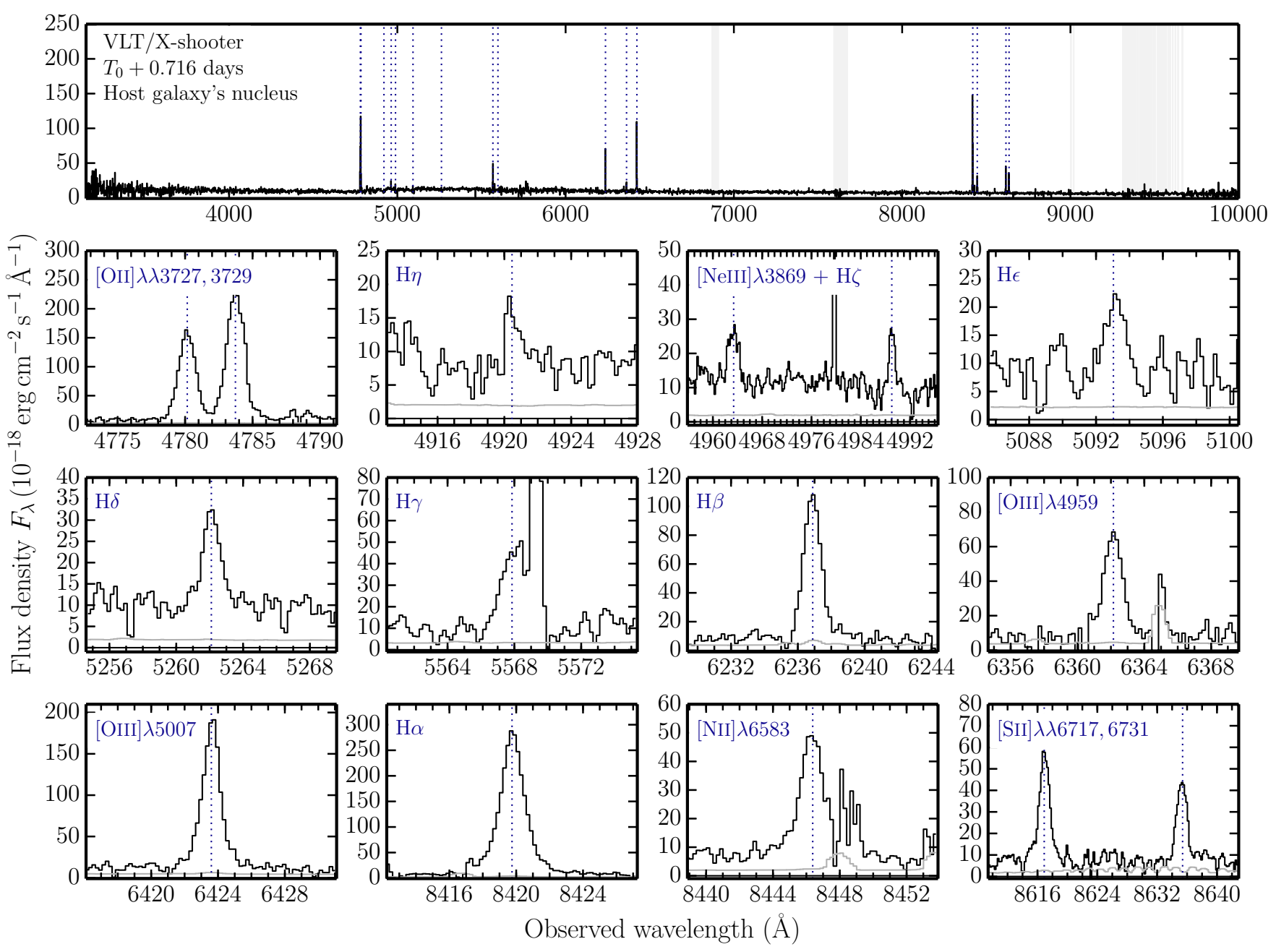

Fig. E.2. Same as Fig. E.1 but for the host galaxy's nucleus. Absorption lines are omitted since none was detected. 